University of Louisville

ThinkIR: The University of Louisville's Institutional Repository

Electronic Theses and Dissertations

$5-2020$

\title{
Converting threats into opportunities predicting medical error reporting behavior.
}

Whitney Thomas Rogers

University of Louisville

Follow this and additional works at: https://ir.library.louisville.edu/etd

Part of the Health Services Administration Commons, and the Patient Safety Commons

\section{Recommended Citation}

Rogers, Whitney Thomas, "Converting threats into opportunities predicting medical error reporting behavior." (2020). Electronic Theses and Dissertations. Paper 3368.

https://doi.org/10.18297/etd/3368

This Doctoral Dissertation is brought to you for free and open access by ThinkIR: The University of Louisville's Institutional Repository. It has been accepted for inclusion in Electronic Theses and Dissertations by an authorized administrator of ThinkIR: The University of Louisville's Institutional Repository. This title appears here courtesy of the author, who has retained all other copyrights. For more information, please contact thinkir@louisville.edu. 


\title{
CONVERTING THREATS INTO OPPORTUNITIES PREDICTING MEDICAL ERROR REPORTING BEHAVIOR
}

By

Whitney Thomas Rogers

B.A., University of Louisville, 1995

M.A., University of Louisville, 2010

MPH, University of Louisville, 2013

\begin{abstract}
A Dissertation
Submitted to the Faculty of the

School of Public Health and Information Sciences of the University of Louisville in Partial Fulfillment of the Requirements for the Degree of
\end{abstract}

Doctor of Philosophy in Public Health Science

Department of Health Promotion and Behavioral Sciences

University of Louisville

Louisville, Kentucky

May 2020 
Copyright 2020 by Whitney Thomas Rogers

All rights reserved 

CONVERTING THREATS INTO OPPORTUNITIES

PREDICTING MEDICAL ERROR REPORTING BEHAVIOR

By

Whitney Thomas Rogers

B.A., University of Louisville, 1995

M.A., University of Louisville, 2010

M.P.H., University of Louisville, 2013

A Dissertation Approved on

April 20, 2020

by the following Dissertation Committee:

A. Scott LaJoie, Ph.D., MSPH

Dissertation Chair

William P. McKinney, M.D.

Cara Cashon, Ph.D.

Jelani Kerr, Ph.D., MSPH

Susan Buchino, Ph.D., OTR/L 


\section{DEDICATION}

This dissertation is dedicated my father

Mr. Anthony D. Thomas Jr.

who has always given me unconditional love and support. You are the best father and teacher and I am forever grateful. Thanks for teaching me how to climb all those hills.

I love you, Dad.

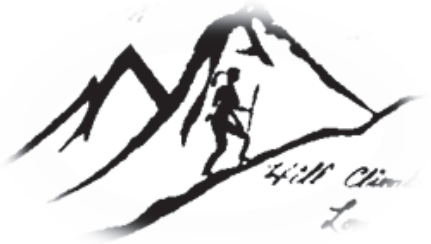




\section{ACKNOWLEDGMENTS}

I owe so much to the people in my life who have contributed to the success of this journey, for without their support this would not have been possible. I would like to first acknowledge the wonderful faculty, staff, and students at the SPHIS that I have had the great pleasure of meeting and working with over the past decade. I have many great memories and look forward to finally being on the other side of the desk and creating many more.

To my committee, thank you for your thoughtful feedback and for suffering through pages and pages of nonsensical ramblings. It's always interesting to read something with fresh eyes, my apologies. To my mentor, Dr. LaJoie (Scott). Thank you for sticking with me all these years and having faith that I would finish. I am certain that I would not have completed this dissertation had I chosen a different mentor. My path to this point has been challenging, yet somehow you found a way to balance moving me through the process while extending grace. Thank you for seeing me and meeting me where I was, not where I should be.

I must also acknowledge the strong leaders that I have had the pleasure of working under during my 17 years as a full-time employee of the University. Thank you, Nigel and Irene, for supporting me and allowing me to complete my education while working. It was not easy juggling school and work, but you trusted me and I am very 
grateful. Cheers!

To all my friends that I love and adore, I would like to take the time to finally answer the question that so many of you have asked me, relentlessly, over the years. Yes, I am done with school. I often say that won the lottery of awesome family and friends and I mean that from the bottom of my heart. I cannot wait to celebrate (post global pandemic quarantine, of course) around all the campfires.

The biggest and most heartfelt acknowledgement goes to my family, who has been by my side the entire time. I know there were many years where you stood on the sidelines cheering for me, without having any clue what game I was playing. Your faith and love and support have seen me through everything in life, this journey included.

To my mother, Karen, who left this world way too early, I wish you were here to celebrate with us. I miss you every day. To my father, who this dissertation is dedicated to. I will always cherish the words you told me as I faced my first big challenge of this journey. No hill for a hillclimber, Whitney Paige. I learned from the best.

To my husband Chris and children Norah and Brighton without whom this dissertation would have been completed 3 years earlier. I love you all so very much. Thank you for your patience and understanding over the years. I know it has not always been easy, but here's hoping it was worth it. Let the adventures begin! 


\begin{abstract}
CONVERTING THREATS INTO OPPORTUNITIES

PREDICTING MEDICAL ERROR REPORTING BEHAVIOR
\end{abstract}

\author{
Whitney Thomas Rogers
}

April 20, 2020

Medical errors are a public health epidemic and a major threat to patient safety. Estimates suggest they may be responsible for 210,000 - 440,000 preventable deaths per year. This would make medical errors the third leading cause of death in the United States. The societal burden of medical errors is steep, with the economic impact near $\$ 1$ trillion. The purpose of this study was to use a theory-driven approach to better understand the reporting behavior of healthcare professional. Increased error reporting leads to heightened awareness of the precursors or conditions generating errors, which can then be corrected. In its most simplistic view, reporting is tool used towards the prevention of medical errors.

A health care marketing firm was hired to recruit 106 health care professionals to complete a survey on their intention to report medical errors. Random and anonymous sampling techniques were employed to help mitigate the risk associated with answering questions that may be risky and lead to social desirability bias. Regression model statistics were statistically significant in explaining $53.3 \%$ of the variance in intention 
scores, $\mathrm{R} 2=.533, \mathrm{~F}(8,69)=9.86, \mathrm{p}<.001$. Constructs of behavioral attitude $(\beta=.537$, $p=.001)$, perceived behavioral control $(\beta=.594, p=.002)$, and actual behavioral control ( $\beta=1.25, p=.005)$, contributed significantly to the model statistics. Psychological safety was not a significant predictor of intention.

These findings suggest psychological safety may be redundant with the theory's existing predictors, working through one or more of the theory constructs. Developing novel strategies for improving the reporting behavior of health care providers, targeting behavioral attitude and perceived behavioral control, will be essential in safeguarding the health of the public through the reduction of medical errors. 
TABLE OF CONTENTS

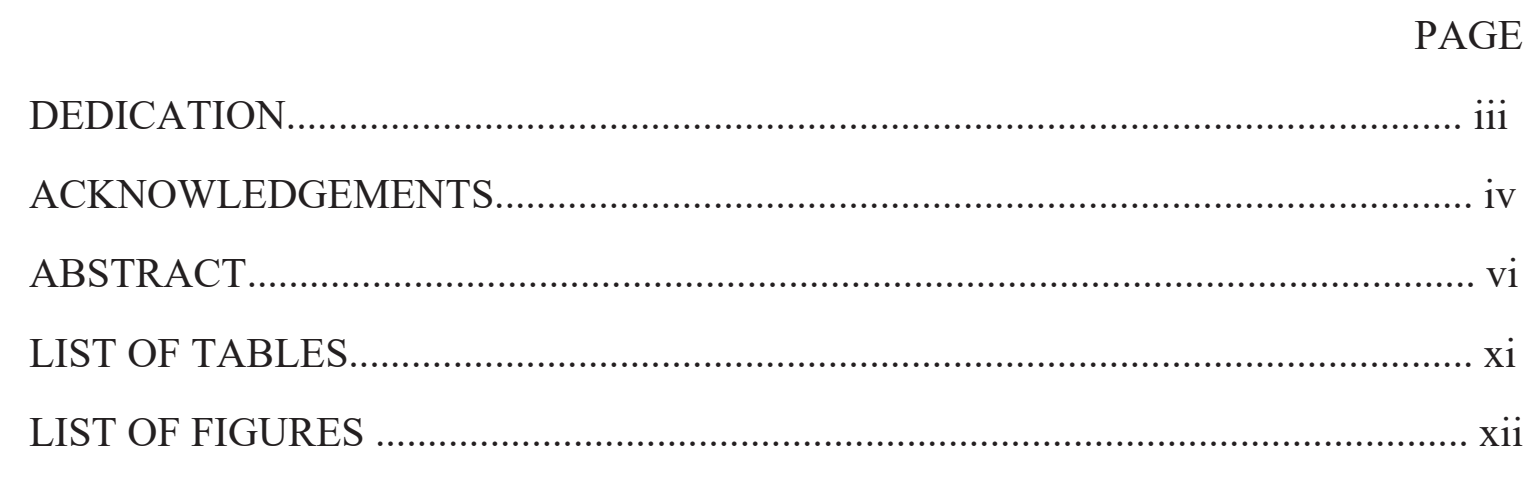

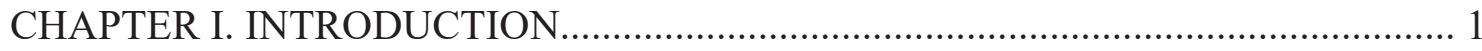

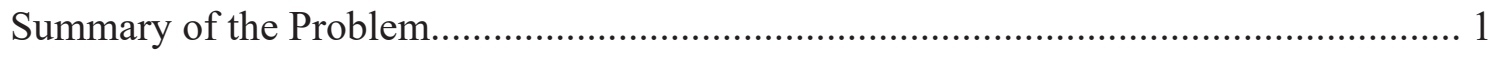

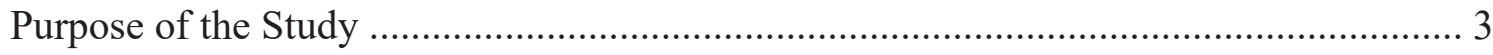

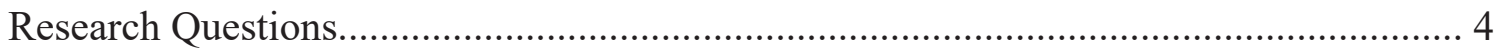

CHAPTER II. REVIEW OF THE LITERATURE......................................................... 7

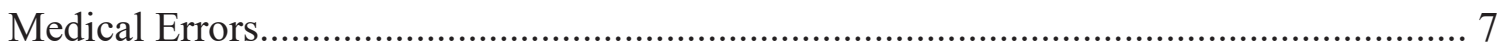

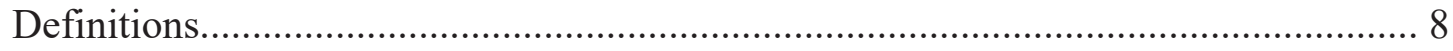

Magnitude and Public Health Significance ................................................................ 8

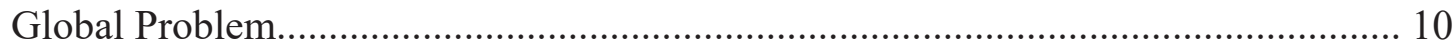

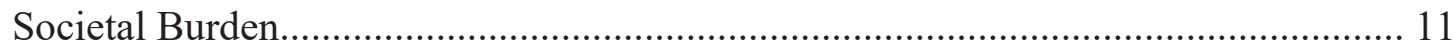

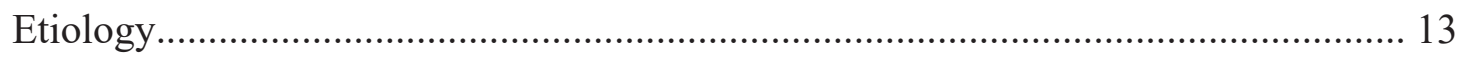

Managing Errors................................................................................................. 16

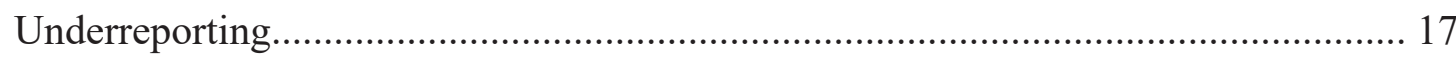

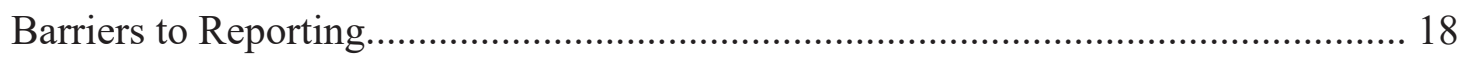

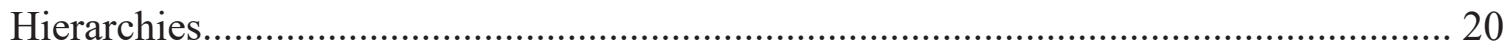

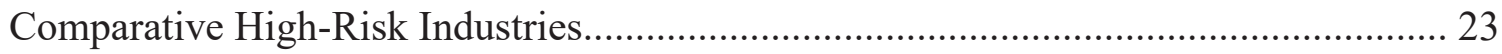




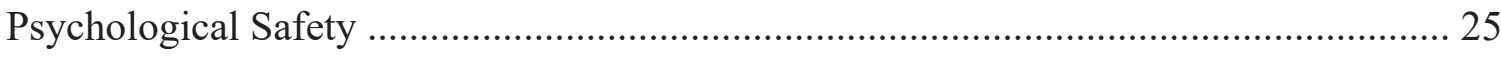

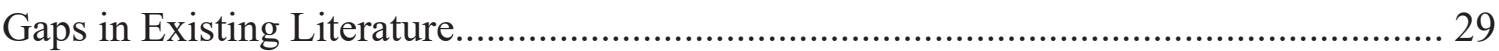

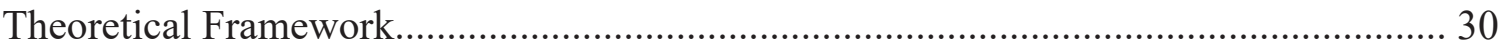

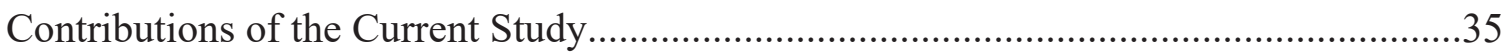

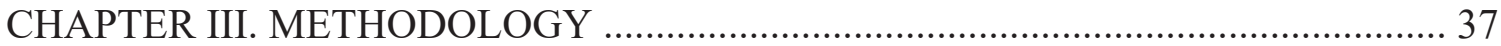

Research Questions and Hypothesis.............................................................................. 37

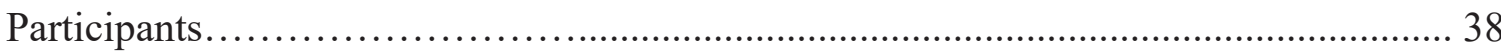

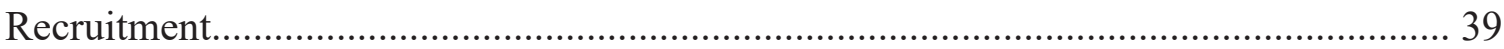

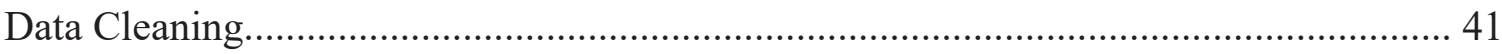

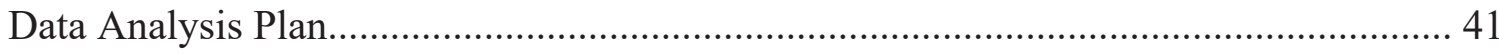

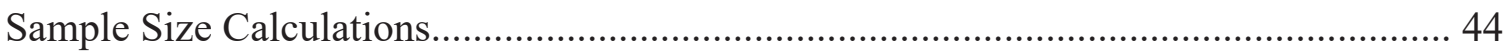

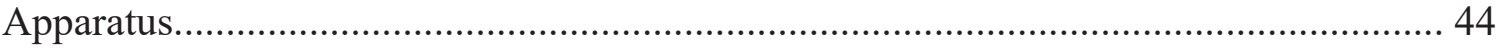

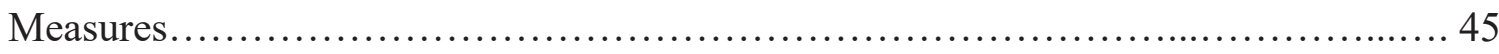

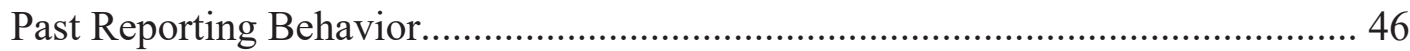

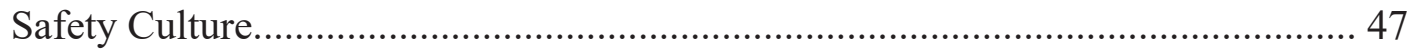

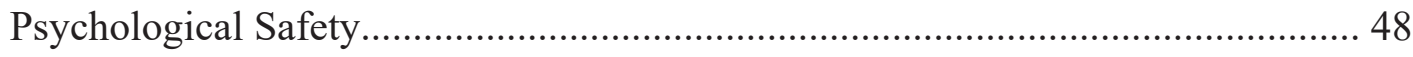

Theory of Planned Behavior..................................................................................... 49

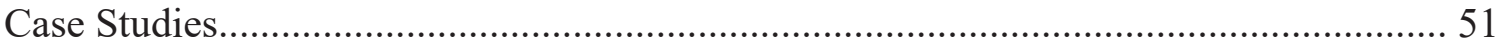

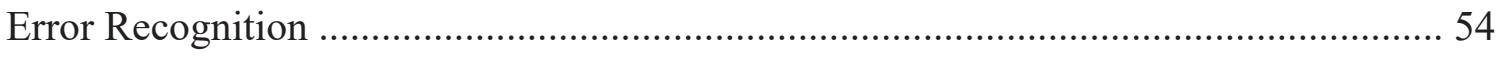

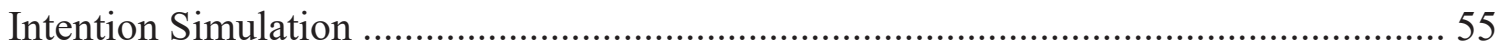

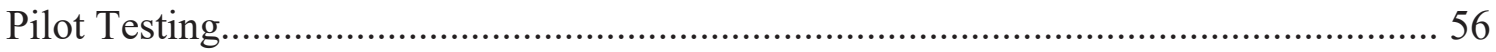

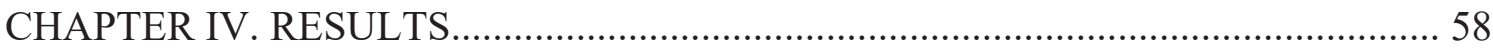

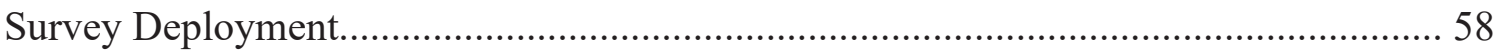

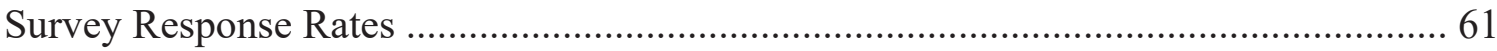

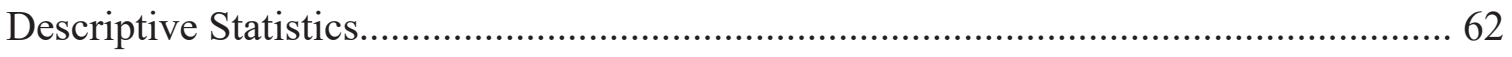

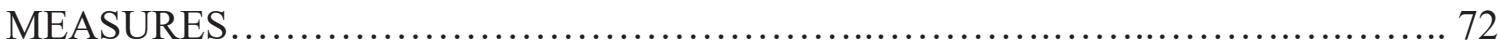

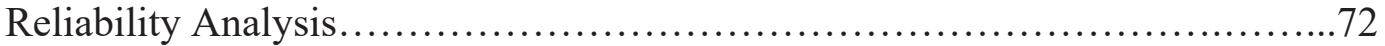


Psychological Safety............................................................ 73

Theory of Planned Behavior................................................... 74

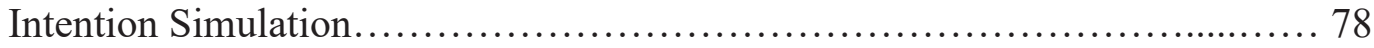

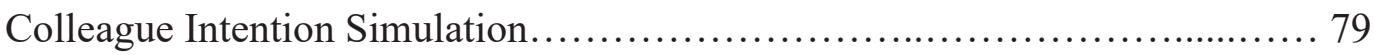

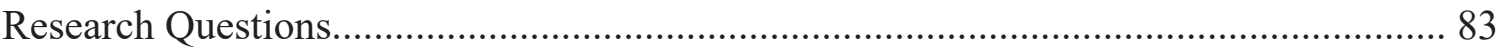

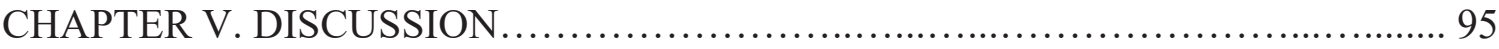

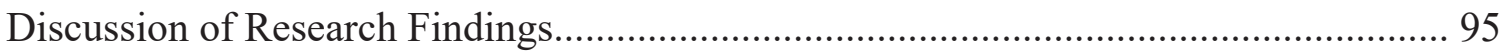

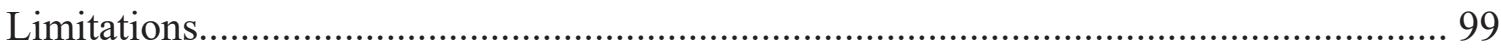

Social Desirability Bias...................................................... 99

Conclusions and Future Directions............................................. 101

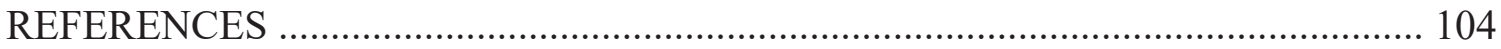

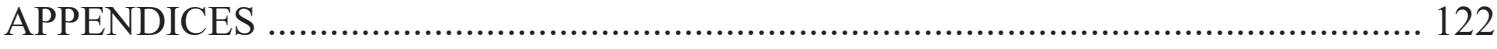

Appendix A: Institutional review board (IRB) approval ............................................. 122

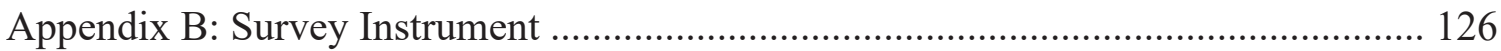

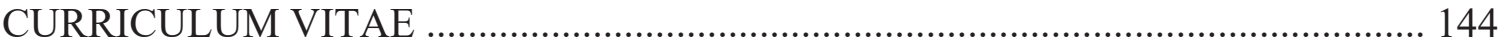




\section{LIST OF TABLES}

Table 1. Demographic Characteristics of Sample

Table 2. Correlations between TPB Constructs \& Intention to Report.................. 77

Table 3. TPB Predictors Summary Scores ............................................................ 78

Table 4. Participant Responses to Case Studies........................................................ 83

Table 5. Correct Decision Scores ............................................................................... 84

Table 6. Results of the Multiple Linear Regression Analyses................................... 86

Table 7. Correlations between Psychological Safety and TPB Constructs .............. 87

Table 8. Correlations between TPB Constructs \& Intention Simulation ....................... 94 


\section{LIST OF FIGURES}

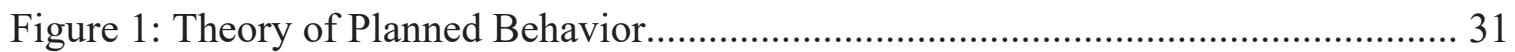

Figure 2: Theory of Planned Behavior, Expanded Model................................................. 34

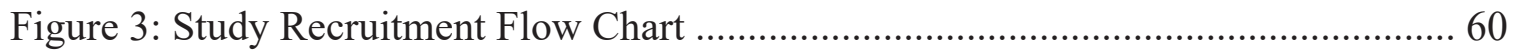

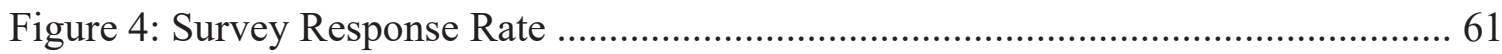

Figure 5: Reporting Behavior: Errors Committed or Observed and Errors Reported...... 69

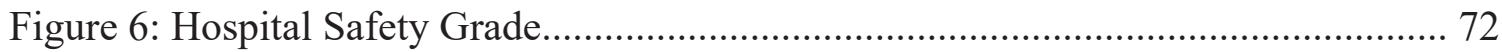

Figure 7: Intention to Report Medical Errors: Perceptions of Self and Colleague ......... 81

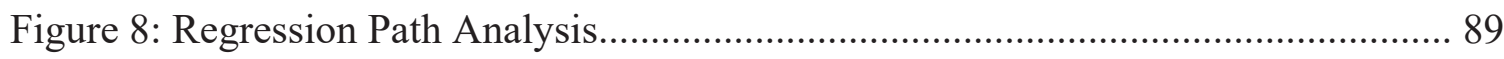




\section{CHAPTER I}

\section{INTRODUCTION}

\section{Summary of the Problem}

Primum non nocere "first do no harm" is a fundamental maxim of medical education. Although this well-known aphorism is often referenced (erroneously) as part of the Hippocratic Oath, it contains a concise and forcefully expressive general truth. One area in which the healthcare system fails to uphold this truism is the capacity to protect patients from harm due to medical errors. Medical errors are a public health epidemic and a major threat to patient safety. Some estimates suggest medical errors could be the third leading cause of death in the United States (Makary \& Daniel, 2016). In their groundbreaking To Err is Human report, the Institute of Medicine (IOM) decreed the epidemic of medical errors a result of unsafe systems, not reckless or incompetent individuals (1999). Recommendations to create safer systems included the implementation of voluntary and mandatory error reporting systems.

Efforts to increase error reporting by health care professionals have been unsuccessful, with widespread paucity of reporting. In 2012, the Health and Human Service's Office of Inspector General (OIG) released a report on hospital incident reporting systems and patient harm. Their analysis of Medicare beneficiaries found 86 
percent of errors were not reported to hospital incident reporting systems (Levinson, 2012). The OIG's report listed lack of education (type of errors that should be reported) and inconsistent application of reporting protocols by employees as key contributing factors. A later study on the implementation of an error reporting system suggested underreporting in health care may be closer to 90 percent (Anderson \& Abrahamson, 2017).

It is widely accepted that medicine is a complex healing art, in which doctors enter into an implicit social contract with their patients. In return, medicine is granted the privilege of self-regulation. Self-regulation is part of the ideology of medical professionalism and requires an ethical obligation to keep its members from harming society. Reporting is at the cornerstone of these efforts. However, the data suggest the profession has been not been successful in honoring this part of the contract (Shojania, Duncan, McDonald, \& Wachter, 2001). If interventions are not successful in improving the reporting behavior of health care professionals, then alternative approaches to regulation are needed.

The barriers to reporting are complex. In a post-IOM follow-up report asking why health care was not safer, cultural barriers (the culture of medicine) was cited as the primary reason for the lack of progress (Leape \& Berwick, 2005). Fear of individual blame, repercussions, liability, and litigation were given as explanations for not reporting. This problem is perpetuated by a deep-seated power structure and a 'blame and shame' culture. Individuals who do not feel safe in their environment will not speak freely about sensitive issues such as errors (Derickson, Fishman, Osatuke, Teclaw, \& Ramsel, 2015). 
A term coined by Edmondson, psychological safety refers to "a shared belief by members

of a team that the team is safe for interpersonal risk taking" (Edmondson, 1999, p. 350).

\section{Purpose of Study}

The broad purpose of this study was to better understand medical error reporting behavior in a random sample of health care professionals licensed in the state of Kentucky. A simplistic view towards the prevention of medical errors is that heightened awareness identifies the mechanisms generating error that can then be corrected.

Data were collected and analyzed using sequential explanatory design (Creswell, Plano Clark, Gutmann, \& Hanson, 2003). In the first sequence, quantitative methods were used to predict reporting behavior using a survey instrument designed to capture the constructs of the theory of planned behavior (TPB). The relationship between the antecedents of behavior (attitude towards behavior, subjective norms, perceived behavioral control) and the intention to report medical errors (a proxy for behavior when observing actual behavior is difficult) were modeled using regression techniques. Psychological safety was added to the model to assess additional explained variance in intention scores.

In the second sequence, responses to case studies were used to "help explain, or elaborate on, results obtained in the first phase" (Ivankova, Creswell, \& Stick, 2006, p. 5). Specifically, responses to the case study scenarios were used to calculate an alternative measure of intention, defined as intention simulation. Intention simulation is described in the Theory of Planned Behavior Questionnaire: Manual for Researchers as a 
"more valid proxy measure of actual behavior" when investigating behavior in health care professionals (Francis et al., 2004, p. 12). This method was designed to elicit complex behavioral decisions based on the presentation of real clinical situations believed to more accurately reflect actual behavior.

In this study, intention simulation was assessed using case studies taken from the Patient Safety in Rural Healthcare Settings study (Cook, Hoas, Guttmannova, \& Joyner, 2004). Participants were asked to read six scenarios and make a reporting decision based on the information provided. Measures of intention simulation and generalized intention (collected in the first sequence) were then compared. By capitalizing on the strengths of each approach, these methods provided a robust analysis of the contextual factors that motivate individual behavior (Greene, Caracelli, \& Graham, 1989; Tashakkori \& Teddlie, 1998). Understanding the factors which both facilitate and inhibit reporting behavior, including the intention to report, is critical to the overall goal of reducing medical errors. The research questions and corresponding hypotheses for this study are:

Research Question 1(a) - What is the relationship between attitude towards behavior, subjective norms, perceived behavioral control and the intention to report medical errors?

Hypothesis 1: The theory of planned behavior will significantly predict the intention to report medical errors.

Research Question 1(b) - Does the inclusion of additional variables in an expanded theory of planned behavior model explain additional variance in 
intention scores?

Hypothesis 2: Inclusion of background variables, past reporting behavior, and actual behavioral control will significantly predict intention scores.

Research Question 2(a) - What is the relationship between psychological safety and the intention to report?

Hypothesis 1: Psychological safety will be a significant predictor of the intention to report medical errors.

Research Question 2(b) - What is the relationship between TPB antecedents of behavior, psychological safety, and the intention to report medical errors?

Hypothesis 2: The inclusion of psychological safety as an additional predictor variable in the theory of planned behavior will significantly improve explanatory power of the model.

Research Question 2(c) - Does the inclusion of psychological safety in an expanded model explain additional variance in intention scores?

Hypothesis 3: Inclusion psychological safety as an additional predictor variable in an expanded model of the TPB will significantly predict intention scores.

Research Question 3 - How does psychological safety influence the intention to report?

Hypothesis 1: Psychological safety will independently influence the 
intention to report.

Research Question 4 - Can an alternative measure of intention better approximate actual behavior?

Hypothesis 1: Intention simulation will serve as a better proxy for actual behavior than generalized intention in predicting error reporting behavior. 


\section{CHAPTER II \\ LITERATURE REVIEW}

\section{Medical Errors}

Defining medical errors is as complex as the environment in which they occur, with most definitions' dependent on the context in which they are being studied. The most commonly cited definition originates from the Institute of Medicine (IOM), which defines medical errors as "the failure of a planned action to be completed as intended (an error of execution) or the use of a wrong plan to achieve an aim (an error of planning)" (IOM, 1999; Reason, 1990).

Lucian Leape, physician and professor who published the classic Harvard Medical Practice Study (HMPS), defined medical errors as an "unintended act (omission or commission) or one that does not achieve its intended outcome" (Leape, 1994, p. 1851). Errors of commission result from action, either in the planning or execution stage, and errors of omission result from a failure to act. As defined by the authors of the HMPS, adverse events are "injuries caused by medical management", rather than events caused by an underlying disease (Brennan et al., 1991, p. 370). To clarify, all negative outcomes are not the result of medical errors (Uribe, Schweikhart, Pathak, Dow, \& Marsh, 2002), and errors that do not result in negative outcomes or adverse events are called near misses (Firth-Cozens, 2002). However, adverse events that do result from 
medical errors are referred to as preventable adverse events. The World Health Organization (WHO) describes errors as incidents or "any deviation from usual care that imposes an injury to the patient or creates a risk of harm; including preventable adverse events and hazards" (WHO, 2005a). The Agency for Healthcare Research and Quality (AHRQ) describes them simply as events (AHRQ, July 2013). The lack of standardized nomenclature and taxonomy, overlapping terms, and surrogate measures make it difficult to obtain epidemiological measures, identify root causes, and develop effective solutions (Grober \& Bohnen, 2005).

\section{Magnitude and Public Health Significance}

The problem of medical errors was brought to the attention of the world in 1999 when the Institute of Medicine (IOM) published the landmark report, To Err is Human: Building a Safer Health System and revealed that medical errors were responsible for between 44,000 and 98,000 preventable deaths every year (IOM, 1999). These estimates were based on the results of the first epidemiological study of medical errors conducted, the Harvard Medical Practice Study (HMPS).

In 1984, the HMPS sought to obtain epidemiological data on adverse events and negligence in a New York hospital population (Brennan et al., 1991). In this study, adverse events were defined as "injury caused by medical management that prolonged hospitalization or resulted in disability" and negligence as "care not meeting the industry standards" (Brennan et al., 1991, p. 145). The HMPS reviewed over 30,000 hospital records and produced an incidence rate of 3.7 percent for adverse events and 27.6 percent for negligence. Although most adverse events resulted in minor impairment (56.8 
percent), 14 percent of these events resulted in death. When applied to the population of New York, the study concluded that over 13,000 deaths were related to adverse events (Brennan et al., 1991). Using the HMPS data, the IOM extrapolated these estimates to the nearly 33.6 million hospitalizations nationwide and concluded over 180,000 individuals in the United States die each year due to injury resulting directly from medical care. The magnitude of this problem is equivalent to "three jumbo-jet crashes every two days" (Leape, 1994, p. 1851).

Further epidemiological studies suggest the problem of medical errors is much greater than what was reported by the IOM. In 2000, data assessing the incidence of adverse events and negligence in Colorado and Utah hospitals were published (Thomas et al., 2000). Utilizing similar methodology to the HMPS, researchers evaluated over 14,700 discharge records from 1992 across 90 hospitals in Colorado and 53 in Utah. Study investigators found adverse events were detected in nearly 3 percent of hospitalizations in both states (Thomas et al., 2000). These results were similar to the incidence and types of adverse events found in New York by the HMPS.

A more recent evidence-based assessment of patient harm concluded the problem to be worse, with estimates between 210,000 and 440,000 preventable deaths per year related to adverse events (James, 2013). In this study, James (2013) systematically reviewed medical records using the Global Trigger Tool (GTT) to capture triggers or clues that a PAE had occurred. Utilizing this methodology, PAEs accounted for an overall death rate of 0.89 percent. Among all adverse events, the percentage of PAEs was 69 percent across all studies (James, 2013, p. 125). Given the 34.4 million hospital 
discharges in 2007, James (2013) concluded 210,000 preventable deaths were related to PAEs, an estimate that did not account for undocumented errors, errors missed by measurement tools, and errors of failing to make a life-saving diagnosis. An earlier study by Weisman et al., (2008) had also revealed serious problems with undocumented errors in medical records and suggested a multiplier be applied to account for these factors. Although Weissman et al. (2008) suggested a threefold increase, James (2013) chose a more conservative factor of two. Using this formula, it was estimated over " 440,000 preventable adverse events contribute to the death of patients each year from care in hospitals" (James, 2013, p. 127).

Ultimately, these statistics suggest death by PAE, or medical errors, is the third leading cause of death in the United States (U.S.) behind heart disease $(647,457)$ and cancer $(599,108)$. According to the 2017 vital statistics report released by the Centers for Disease Control and Prevention, National Center for Health Statistics (2019) the next closest cause of death is accidents/ unintentional injury $(169,936)$, followed by chronic lower respiratory disease $(160,201)$. Even using the more conservative estimates cited in the IOM (1999), medical errors are the seventh (98,000 deaths per year) or eleventh (44,000 deaths per year) leading cause of death in the United States.

\section{Global Problem}

Patient safety and medical errors are not unique to the United States. Estimates from a detailed review of 1000 case files in two London hospitals found 10 percent of all hospital admissions resulted in an adverse event, 5.2 percent of which were PAEs (Vincent, Neale, \& Woloshynowych, 2001). Extrapolating these figures across the 15.1 
million hospital admissions reported by National Health Service (NHS) in England from 2012-2013 resulted in estimates of approximately 755,000 PAEs (Economics, 2014).

The Australian health system also reported a "silent epidemic" of errors. With 18,000 deaths and 50,000 permanent injuries attributable to medical errors, approximately 1 in 6 (16.6 percent) of patients in Australian hospitals have been reported to suffer adverse or harmful events (Robinson et al., 2002; WHO, 2005b). Canada, New Zealand, and Denmark are also facing challenges with medical errors, making this a global public health issue. In 2002 during the $55^{\text {th }}$ World Health Assembly, WHO designated patient safety as a public health priority and established a World Alliance for Patient Safety. WHO member states were urged to promote safety as a "fundamental principle of all health systems" out of concern that the incidence of adverse events was "a challenge to quality of care, a significant cause of human suffering, and a high toll in financial loss and opportunity cost” (WHO, 2002, p. 1).

$\underline{\text { Societal Burden }}$

The societal burden of medical errors is steep and rising. In 1989, the annual cost attributed to medical injuries (total health and productivity loss) in a New York State hospital was estimated to be $\$ 878$ million (Johnson et al., 1992). In 1996, a similar study conducted in hospitals across Utah and Colorado found costs attributable to adverse events (additional medical expenses and lost productivity) to be $\$ 662$ million, of which \$308 million were directly related to medical errors (Thomas et al., 1999). When these figures were extrapolated to all U.S. hospital admissions, the annual costs were projected at $\$ 37.6$ billion for adverse events and \$17 billion for medical errors. Using 2008 
population data and estimates from the 1989 New York State study, the cost of medical injuries were projected at nearly $\$ 50$ billion (Van Den Bos et al., 2011).

Until recently, the annual economic impact of medical errors in the U.S. was in the range of $\$ 17$ to 50 billion using the methodology described above. However, a 2010 study sponsored by the Society for Actuaries and conducted by an independent actuarial and consulting firm (Milliman) found the economic impact of preventable medical errors may be closer to $\$ 1$ trillion when quality-adjusted life years (QALY) were taken into account (Andel, Davidow, Hollander, \& Moreno, 2012). The authors present a best-case scenario using IOM estimates of 98,000 preventable deaths, with an average of 10 "lost life" years at a rate of $\$ 75,000$ to $\$ 100,000$ per year. Using these conservative parameters, Andel et al. (2012) estimated $\$ 73.5$ to $\$ 98$ billion loss from QALYs lost. Acknowledging the IOM estimates may be grossly understated, with some reports suggesting deaths due to medical errors could be 10 times higher than reported, the authors suggest the economic impact or burden of medical errors may, in actuality, be closer to $\$ 735$ to $\$ 980$ billion annually.

The societal burden of medical errors is a shared global problem. Data from the United Kingdom (UK) also suggest a significant economic impact due to medical errors. A 2014 report prepared by Frontier Economics for the National Health Service (NHS) explored the cost of unsafe care. Using extrapolation, they reported an annual cost of $£ 2.5$ billion related to PAEs, most of which were related to medication errors (Economics, 2014). 


\section{$\underline{\text { Etiology of Errors }}$}

The etiology of medical errors is complex (Alsafi et al., 2011; Poorolajal, Rezaie, \& Aghighi, 2015) and errors are inevitable in certain systems (Perrow, 1994). Humans are inherently error-prone, and healthcare delivery systems are fragmented and complex. The convergence of these factors creates the conditions that make errors more likely to occur. There are many types of errors that can occur during the course of delivering health care, ranging from widely publicized cases, such as Willie King's incorrect surgery site that resulted in a wrong leg amputation (R. I. Cook, Woods, \& Miller, 1998), to lesser publicized errors resulting in falls, burns, hospital-acquired infections, pressure ulcers, and medication errors (IOM, 1999). Unlike errors that occur in other high-risk industries (e.g., Nuclear Power/Three Mile Island; Aviation/Challenger Mission), medical errors occur at the individual level and rarely make newspaper headlines.

The IOM adopted a classification system based on Leape's HMPS that categorized errors into groups based on the type of error, such as diagnostic, treatment, preventive, or other (Leape, Lawthers, Brennan, \& Johnson, 1993). Diagnostic errors include "delays in diagnosis; failure to employ indicated tests; use of outdated tests or therapy; and failure to act on results on monitoring or testing" (IOM, 1999, p. 36; Leape et al., 1993). Treatment errors encompass "error in the performance of an operation, procedure or test; error in administering the treatment; error in the dose or methods of using a drug; avoidable delay in treatment or in responding to an abnormal test; and inappropriate (not indicated) care" (IOM, 1999, p. 36; Leape et al., 1993). Preventive errors cover "failure to provide prophylactic treatment and inadequate monitoring or 
follow-up treatment" (IOM, 1999, p. 36; Leape et al., 1993). Other errors combine "failures of communication; equipment failure; and other system failures" (IOM, 1999, p. 36; Leape et al., 1993).

Within each category arise errors associated with misuse and overuse (i.e., errors of commission) and underuse (i.e., errors of omission) of medical care. Errors of omission pose a risk of preventable death through suboptimal care, while errors of commission pose risk from interventions that failed to achieve their expected outcomes or those that were simply the wrong intervention (Hayward, Asch, Hogan, \& et al., 2005). Errors of commission include injuries resulting from improper execution and planning of treatment. James Reason, a pioneer in the field of human error, described these as errors of execution and errors of planning (Reason, 1990). A planned action that does not proceed as intended is considered an error of execution and encompasses terms such as slips and lapses. Errors of planning involve mistakes, where the action selected was wrong, and therefore cannot achieve its intended outcome (IOM, 1999).

According to Normal Accident Theory, the majority of accidents (between 60 and 80 percent) in any industry involve human error and are preventable (Perrow, 1994). The HMPS found over 70 percent of errors were preventable, with most occurring in highly technical surgical subspecialties and complex environments, such as emergency departments, operating rooms, and intensive care units (Leape et al., 1993). A study of adverse events in hospitals across Utah and Colorado found 52.6 percent of preventable errors occurred in emergency departments (Thomas et al., 2000). A September 2019 patient safety update from WHO reported that one out of every 10 patients treated in 
high-income countries experience harm as a direct result of being hospitalized, of which 50 percent were preventable. Globally, the problem is much worse in low- and middleincome countries, where rates of preventable harm are closer to 80 percent (WHO, 2019).

There are numerous ways in which human error contributes to preventable accidents. Reason describes active errors as those that occur at the operator level or are directly controlled by the operator (Reason, 1990). Active, also known as sharp-end errors, typically have a direct and immediate impact (R. I. Cook et al., 1998). A pilot crashing a plane or a surgeon amputating the wrong leg are examples of active errors (IOM, 1999). Unlike active errors, latent, or blunt-end, errors occur outside of the control of the operator (R. I. Cook et al., 1998). Latent errors are frequently related to defects or malfunctions in equipment or organizational processes and often go undetected. In aviation, one example of a latent error is a design flaw that gives rise to steering misalignment of an aircraft. Latent errors receive the least amount of attention and have the potential to cause the most damage, as they often remain in the system undetected, giving rise to numerous active errors (IOM, 1999). The IOM reported the investigation of the Challenger explosion found latent errors that had been present nine years before the accident.

In contrast to Natural Accident Theory, High-Reliability Theory states that "serious accidents with hazardous technologies can be can be prevented through intelligent organizational design and management” (Sagan, 1993, p. 2). This safety orientation is echoed in the IOM report, which concluded certain conditions create errors that go beyond individual responsibility (IOM, 1999). This systems approach to 
preventing errors and improving patient safety requires modification to the conditions that contribute to or create errors. Reason (1990) describes these as precursors or preconditions. Precursors or conditions that lead to errors include latent factors such as work schedules, training, and properly working equipment.

\section{Managing Errors}

Reporting is a means of identifying the precursors or preconditions to errors. It provides information on where errors occur, the conditions under which their risk of occurrence is increased, and opportunities for improvements (Kronman, Paasche-Orlow, \& Orlander, 2012). Once an error is identified, an analyst can identify the root causes (root cause analysis) or conditions leading up to the incident, so that corrective action plans can be implemented. Without this type of formal data, opportunities to improve patient safety are hindered. Because reporting converts threats into learning opportunities (Poorolajal et al., 2015), it is a critical behavior in error management and fundamental to the broad goal of error reduction (Hartnell, MacKinnon, Sketris, \& Fleming, 2012).

The IOM recommended both mandatory and voluntary error reporting to help reduce medical errors by 50 percent over five years (IOM, 1999). Mandatory reporting systems were recommended for serious adverse events, such as those resulting in severe harm or death, and voluntary systems for those causing minimal harm, such as near misses. The most recent survey conducted by the National Academy for State Health Policy (NASHP) on compliance with the IOM recommendations found 27 states with active (adverse event) reporting systems, one in the implementation stage, and 23 states verifying no active systems (Hanlon, Sheedy, Kniffin, \& Rosenthal, 2015). 
Reporting and reporting systems are also a requirement of accreditation agencies, such as Joint Commission on Accreditation of Healthcare Organizations (JACHO), an organization which certifies over 21,000 health care organizations and programs in the United States (The Joint Commission, 2020). These accreditations confer a commitment to quality and patient safety in health care and include robust reporting systems and supportive leadership. As outlined in JACHO's Comprehensive Accreditation Manual for Hospitals, individuals in these organizations "recognize all patient safety events [from slips and near misses to serious adverse events resulting in death] must be reported" (Patient Safety Chapter, 2017, p. 4). These standards are consistent with IOM recommendations that reporting is critical to managing and preventing errors. Despite the emphasis on error reporting from both the IOM and major accreditation agencies, the majority of errors go unreported (Appelbaum, Dow, Mazmanian, Jundt, \& Appelbaum, 2016).

\section{Underreporting}

Underreporting is a substantial problem undermining efforts to eliminate medical errors (Antonow, Smith, \& Silver, 2000; Gunn, 2000; Wu, 2000). Underreporting of errors occurs across settings and disciplines; in a study of high-risk (non-health related) industries, it was found that for every adverse event reported, 2.48 events went unreported (Probst \& Estrada, 2010). An AHRQ report, "Critical Analysis of Patient Safety Practices," suggests even higher rates of underreporting in health care (Shojania et al., 2001). A 2005 study conducted at a Boston teaching hospital compared patientidentified adverse events against medical records and incident reporting systems. 
Patients identified 310 "distinct incident reports," 75 percent of which $(n=235)$ were substantiated by independent physician reviewers. However, medical records provided evidence of 62 documented events, and yet no events were reported to the hospital's incident reporting system (Weingart et al., 2005).

O'Neil and colleagues conducted a similar review of medical records and found only 1.5 percent of adverse events were formally reported (O'Neil et al., 1993). Other studies have found up to 96 percent of medical errors go unreported (Barach \& Small, 2000). In 2010, the Department of Health and Human Services (DHHS) Office of the Inspector General (OIG) reported similar findings, with 93 percent of adverse events going unreported. Of the seven percent of reported errors, only two percent were found to be accurate (Levinson, 2010). A 2012 follow up report from the DHHS revealed health care workers did not report patient harm six out of seven times (Levinson, 2012). These studies reveal the majority, if not all, medical errors go unreported and problems with reporting are substantial.

\section{Barriers to Reporting}

Barriers to reporting are multifaceted and can be attributed to organizational, individual, and reporting system factors (Barach \& Small, 2000; Pfeiffer, Manser, \& Wehner, 2010). The institutional (organizational) culture in which individuals are immersed has a major impact on reporting behavior (Fein et al., 2005). In their study of high-risk industries, Probst and Estrada (2010) found the organizational safety climate of an organization, defined as "a unified set of cognitions [held by workers] regarding the safety aspects of their organization" (p. 1439), was related to both the number of 
accidents as well as the rate of reporting. Organizations with a strong safety climate experienced both fewer errors and less underreporting, while those with a poor safety climate experienced the opposite (Probst \& Estrada, 2010). In health care, this is referred to as the 'culture of medicine' and is reported to be a significant barrier to reporting (Leape \& Berwick, 2005; Waring, 2005).

Often described as a system of 'blame and shame,' the culture of medicine breeds fear of individual culpability and retribution. Individuals are disinclined to report errors due to deep-seated fears they will be held personally accountable for any errors. These fears are also evident in perceptions and attitudes towards the characteristics of incident reporting systems. A theoretical review article of incident reporting behavior found nearly half (42 to 46 percent) of reasons given for not reporting were linked to fears of legal consequences and an overall absence of organizational support for reporting, such as lack of active leadership and unclear lines of reporting (Pfeiffer et al., 2010).

Individual beliefs regarding the purpose and utility of reporting systems are another barrier to error reporting. A European study on physician incident reporting behavior found high skepticism (Waring, 2005). Physicians expressed beliefs that the health care system was overly complex and uncertain, and humans were inherently errorprone. These factors lead physicians to the believe errors are inevitable and unmanageable and therefore, reporting pointless. Physicians in this study also perceived incident reporting as burdensome and the data it generates as threatening. Confidence was low among participants that reporting could overcome these barriers to improve outcomes. Another study of physician reporting behavior found procedural barriers 
related to incident reporting systems and reporting. Nearly half of participants (45

percent) did not know how to report, and close to 60 percent did not know what kinds of errors to report (Kaldjian et al., 2008).

The literature supports the strong connection between an unsupportive organizational climate and underreporting. In a study of health care workers, focus group participants expressed "profound fears" associated with error reporting. Barriers included fear of retribution (loss of job, hours, and pay), disciplinary action, increased litigation, damage to professional reputation, and unsupportive colleagues (Fein et al., 2005). Fearbased barriers also include the negative experience of disclosing the error to the family and medical team, which elicited feelings of shame. Focus group participants also admitted being fearful that disclosure would "undermine [their] relationship with the patient" and lead to an erosion of trust (Fein et al., 2005, p. 490). A lack of institutional tolerance and support for errors were cited as primary drivers of these fears. $\underline{\text { Hierarchies }}$

Embedded within organizations, status or hierarchies are another barrier to reporting (Lawton \& Parker, 2002; Waring, 2005). Status refers to the degree of respect and influence conferred to an individual based on characteristics such as education, wealth, age, or profession (Nembhard \& Edmondson, 2006). A seminal paper on status describes how individuals possessing more of these attributes are deemed to be more desirable than others, and therefore retain a higher position within the social hierarchy (Benoit-Smullyan, 1944). In the workplace, status is role-based and can be inferred from occupational categories; in the health care industry, there is a well-established 
professional hierarchy, both within and between teams (Kanes, 2010). For example, it is widely acknowledged that surgeons receive higher prestige than do primary care physicians, who in turn garner more prestige than nurses. Likewise, charge nurses retain a higher hierarchical position than floor nurses, and nurses more position than therapists. This preferential treatment leads to feelings of superiority/inferiority, and is known to influence behavior (Tajfel \& Turner, 2004).

It follows, then, that individuals of lower status tend to withhold information, defer decisions, limit behavior, speak less than their higher-status colleagues, and often have lower self-efficacy (Driskell \& Salas, 1991; Leonard, Graham, \& Bonacum, 2004; Pagliari \& Grimshaw, 2002). Kaiser Permanente, the nation's largest nonprofit health plan, reported low-status individuals are less likely to speak up or question higher status individuals (Leonard et al., 2004). A study of fourth-year medical students found a similar "steep authority gradient." The authors discovered that, although the students recognized errors and were supportive of patient safety initiatives, the relationships of "power and social influence undergirding the traditional authority gradient in the culture of medicine" were a major barrier to reporting (Wetzel, Dow, \& Mazmanian, 2012, p. 222). One-quarter of students stated they would never report a faculty member for unsafe behavior, and of the students who did endorse reporting, the majority (91.6 percent) only disclosed errors to residents instead of faculty or staff. Similar results were found in a study of Taiwanese nurses, in which 45 percent of the variance in error reporting behavior was related to differences in status (Chiang \& Pepper, 2006). Reporting across status boundaries is often described as 'whistleblowing' and considered a "cultural 
taboo" (Waring, 2005).

The effect of hierarchies is most evident in industries with poor organizational climates, as evidenced by comparative studies of health care and aviation (Kilduff, Willer, \& Anderson, 2016; Okuyama, Wagner, \& Bijnen, 2014). The Texas Human Factors Group conducted a study on the perceptions of teamwork, communication, hierarchy, errors, and stress by sampling physicians, nurses, and residents ( $\mathrm{n}=1033)$ working in the intensive care unit (ICU) and operating room (OR) and $(n=30,000)$ cockpit crew members (Sexton, Thomas, \& Helmreich, 2000). The researchers found a significant difference in perceptions of hierarchies between the two groups. A majority of cockpit crew members (97 percent) and ICU staff (94 percent) advocated for flat hierarchies, while more than half ( 55 percent) of surgeons believed junior team members should not question the decisions of senior members (p. 747). These hierarchies also impacted teamwork and communication on the health care teams, most notably in the subsample of operating room staff. Sexton et al., (2000), found only 40 percent of nurses reported good teamwork and communication, compared to 77 percent of physicians. This finding supports other research demonstrating troubled relationships between medicine and nursing (Greenfield, 1999; Kaissi, 2008; Zwarenstein \& Bryant, 2000).

The impact of hierarchies has a direct influence on behavior. Trained rater observations of commercial flights and randomly selected medical procedures found 85 percent of flights observed had outstanding to standard ratings of teamwork, compared to an average of 60 percent of the medical procedures observed (Sexton et al., 2000). Another comparative study examined attitudes toward safety, finding problematic or 
oppositional responses (to safety) in 17.5 percent of hospital personnel, compared to only 5.6 percent of naval aviators (Gaba, Singer, Sinaiko, Bowen, \& Ciavarelli, 2003). Hierarchies effect the dynamics of teams, which influence attitudes that, in turn, shape behaviors. These factors may be implicit in the rate and severity of errors founds in the health care industry (B. Sexton et al., 1998; Wiener, Kanki, \& Helmreich, 1993).

\section{Comparative High-Risk Industries}

Many safety lessons can be learned from other high-risk industries. Like aviation and nuclear power, health care organizations are high-risk professions where error has the potential for fatal consequences (Gaba et al., 2003; Lyndon, 2006; Rochlin, 1999). Each of these high-risk industries depend on human behavior to execute daily operations, and are increasingly technical, progressively complex, and stressful. Through concerted efforts to improve communication and teamwork, the aviation industry has been able to transform its safety record (Bates et al., 1997; Kapur, Parand, Soukup, Reader, \& Sevdalis, 2016). The creation of the Occupational Health and Safety Administration (OSHA) in 1971 reduced overall hazardous workplace deaths from "38 worker deaths a day in 1970 to 13 a day in 2015" (Occupational Safety and Health Administration (OSHA), 2017). Although the healthcare industry has adopted of a few of aviation's system-level strategies, such as checklists and standardization, it has still failed to achieve the level of success experienced by other industries (Halasyamani et al., 2006; Horwitz, Krumholz, Green, \& Huot, 2006).

The literature suggests aviation's success in transforming its safety record can be attributed to a united effort to improve the culture of safety through an emphasis on 
communication (Chassin, 2013; Chassin \& Loeb, 2013; Patankar \& Sabin, 2008).

Acknowledging the need for "improved communication, leadership, and decision making in the cockpit," NASA developed a team training concept called Crew Resource Management (Lyndon, 2006; Salas, Burke, Bowers, \& Wilson, 2001; Salas, Wilson, Burke, \& Wightman, 2006). The techniques of CRM create effective teams by establishing a sense of collective agency, where all team members solicit feedback, provide their opinions and recommendations, and speak freely about operations, in order to maintain safety, regardless of hierarchy or role (Rochlin, 1999; Simpson \& Knox, 2003; Sundar et al., 2007). Building on normal accident theory (NAT), in which accidents are regarded as inevitable and managed through active efforts to seek out and avert potential sources of harm (Tamuz \& Harrison, 2006), safety is achieved by collective and continuous efforts to identify and manage sources of harm through effective or assertive communication (Henneman \& Gawlinski, 2004; Simpson \& Knox, 2003). The aviation industry's success using CRM as a model of safety has been cited as a driving force behind health care's increased interest to improve communication and teamwork as an error reducing strategy (Lyndon, 2006).

Assertive communication, speaking up at critical moments or with persistence until a resolution is achieved, is important in error reporting, and known to improve performance (Lyndon, 2006; Lyndon et al., 2012; Simpson \& Knox, 2003). It is considered an essential component of teamwork and plays a critical role in safety, particularly in high-risk industries (Simpson \& Knox, 2003). A retrospective study of over 300 civilian flight report records found a lack of assertive communication 
responsible for the fact that 20 percent of captain errors were unreported by the first officer (Jentsch, Barnett, Bowers, \& Salas, 1999). Simpson and Knox found a similar theme in their review of adverse perinatal events, where lack of assertive communication was associated with increased adverse outcomes and near-misses (Simpson \& Knox, 2003).

The advances in safety demonstrated by the aviation industry were achieved through the cultivation of a culture in which hierarchies are flat, and team members feel safe to speak assertively and openly without fear or repercussion. Aviation achieved this by creating a psychologically safe environment. In order to improve error reporting and ultimately reduce medical errors, the health care industry must follow the example of the aviation industry, and make "substantial changes to achieve a safety climate consistent with the status of high-reliability organizations," (Gaba et al., 2003, p. 173).

\section{$\underline{\text { Psychological Safety }}$}

Some of the most frequently cited reasons for not reporting are linked to fear of repercussions, embarrassment, and professional shame; characteristics of a psychologically unsafe environment (Pfeiffer et al., 2010; Robinson et al., 2002; Waring, 2005). Schein and Bennis (1965) first described the construct of psychological safety in the field of organizational change research where they recognized that individuals must feel secure to implement a change (Fairchild \& Hunter, 2014). Edmondson (1999) defined this group-level construct as "a shared belief by members of a team that the team is safe for interpersonal risk taking" (p. 350). Edmondson characterizes interpersonal risk-taking as "learning" behaviors such as seeking feedback, experimentation, and 
discussing errors. It is through this process that errors, which provide valuable information on processes and procedures that did not work as planned, are discovered and corrected (Edmondson, 1999). Mutual trust and respect among team members create an environment in which individuals feel safe from embarrassment, rejection, and punishment, should they speak up, disagree, or challenge others' views. Individuals working in psychologically-safe environments can express themselves without fear of negative consequences (Appelbaum et al., 2016). Psychologically safe environments promote learning outcomes, such as improved performance and effectiveness, by mitigating concern over how others will react to these learning behaviors.

Although a majority of the literature on psychological safety is centered on organizational team learning, the evidence suggests this construct may play a critical role in understanding the 'motivators for and barriers to' reporting behavior (Appelbaum et al., 2016; Derickson et al., 2015). A Veterans Health Administration (VHA) study found intention to disclose errors was significantly higher in individuals from psychologically safe environments, compared to those from unsafe environments (Derickson et al., 2015). Psychologically unsafe environments were also found to hinder reporting in a mixed sample of nurses and physicians. Respondents reported fear of repercussions and lack of confidentiality as barriers to reporting across all job categories (Jeffe et al., 2004). A study of neonatal intensive care units (NICU) demonstrated psychological safety to be a key factor in staff engagement in quality improvement activities, even after accounting for an overburdened workforce (Nembhard \& Edmondson, 2006). NICU team members reported increased interest and adherence to quality improvement efforts when their 
teams were characterized by "interpersonal trust and respect" (Nembhard \& Edmondson, 2006, p. 957).

Hierarchies are one determinant of psychological safety. A study on the effects of professional status on psychological safety in NICUs confirmed professional status was positively related to psychological safety: physicians reported increased psychological safety compared to nurses, and nurses more than respiratory therapists (Nembhard \& Edmondson, 2006). These self-censoring behaviors, described as organizational silence, are directly related to perceptions of risk. Researchers found that individuals were willing to speak up when they were not worried about disapproval or negative consequences (Detert \& Edmondson, 2005; Edmondson, 2003). They also discovered that individuals would self-censor or refrain from speaking up when leaders employed an authoritarian or autocratic leadership style (Leonard, Graham, \& Bonacum, 2004). These counterproductive communication patterns are most pronounced across status gradients and result in negative tensions that often spill over into aspects of patient care (Park, 2004). This impact on patient care becomes reflected in the incredibly high error rate (70 percent), ultimately attributed to communication failures (Dingley, Daugherty, Derieg, \& Persing, 2008).

The behavior of a team leader has a strong influence on psychological safety and may reduce negative perceptions associated with error reporting (Appelbaum et al., 2016; Edmondson, 1996). Individual team members look to leaders to establish normative behavior regarding what is expected and acceptable in the workplace (Tyler \& Lind, 1992). Autocratic leaders are often perceived as unsafe and threatening, whereas a 
democratic leadership style solicits input from individuals and is perceived as safe and welcoming.

In a study of medication errors, nurses with autocratic managers were hesitant to report or disclose errors out of fear of repercussions, while those with democratic managers reported feeling safe when speaking up (Edmondson, 1996). A study of cardiac teams also found that a democratic leadership style was associated with higher ratings of psychological safety (Edmondson, 2003). Leaders who solicited input from other team members were perceived as psychologically safe, compared to those who were more authoritarian.

A cross-sectional study of Canadian health care workers explored nurses' and physicians' fear of repercussions for reporting medical errors. Leadership behavior at the organizational and unit levels explained 27.6 percent of the variance in fear of reporting for physicians and 15.8 percent for nurses (Castel, Ginsburg, Zaheer, \& Tamim, 2015). It is the combination of low-status individuals working within rigid hierarchies, in environments with low psychological safety, that is believed to be a key contributor to errors and the epidemic of underreporting (Park, 2004; Nembhard \& Edmondson, 2006). Underreporting and Self-Regulation

Underreporting is particularly problematic when it occurs in a self-regulating industry such as health care. Freidson's landmark report (1970) provides an extended analysis of the profession of medicine, which is both autonomous and self-directing. Self-regulation is described as one of the basic tenets of the profession of medicine, for which it is provided a special status of "considerable freedom from outside control" 
(Collier, 2012; Donaldson, 2008, p.1). This freedom infers a social contract based on the principles of altruism, the assertion that the practice requires skills and knowledge not available to non-professionals, and the commitment to regulate its members (Berwick, 2015; Baron, 2015; Cruess, Cruess, \& Stinert, 2009; Donaldson, 2008). The right and obligation of self-regulation is designed to serve and protect patients. It is a privilege and a burden shared by all physicians and must be supported by all members of the profession" (Collier, 2012, p. 1560). Self-regulation works on the understanding that professionals will monitor and discipline themselves and other colleagues in a manner aligned with industry standards (Cruess, Cruess, \& Stinert, 2009). The data on underreporting suggest the profession of medicine, and perhaps the health care industry as whole, is failing to uphold this part of the social contract.

\section{Gaps in Existing Literature}

More than 20 years have passed since the IOM brought the problem of medical errors to the attention of the nation. Despite some progress, the incidence of errors and harm has continued to increase (Adler et al., 2018). Although studies have demonstrated that health care providers acknowledge the importance of reporting, the translation of beliefs into behaviors remains elusive (Kaldjian et al., 2008). This is evidenced by claims that up to 96 percent of medical errors go unreported (Barach \& Small, 2000) and preventable deaths due to medical errors may be the third leading cause of death in the United States (James, 2013).

A critical review of the literature provides some insight on why health care remains so far behind other high-risk industries in safety improvements. One notable gap 
is an absence of theoretically grounded literature on error reporting (Holden \& Karsh, 2007; Karsh, Holden, Alper, \& Or, 2006; Kingston, Evans, Smith, \& Berry, 2004). There is also an overabundance of studies on the barriers to reporting, with limited examination of the predictors of the behavior (Antonow et al., 2000; Beasley, Escoto, \& Karsh, 2004; Leape, 2002; Suresh et al., 2004; Uribe et al., 2002; Wakefield et al., 1999; Weingart, Callanan, Ship, \& Aronson, 2001).

The majority of the theoretical and atheoretical literature on error reporting behavior is centered on medication and adverse drug events, with few studies looking at reporting behavior in a more general context. There is strong evidence to support a theoretically based approach to understanding error reporting behavior. Two recent studies on adverse drug event reporting found the theory of planned behavior (TPB) explained between 16 and 44 percent of the variance in the nursing staff's intention to report (Angelis et al., 2017; Hung, Chu, Lee, \& Hsiao, 2016). A study utilizing the TPB in a sample of hospital pharmacists found the model explained 32 percent of the variance in the intention to report medication errors (Williams, Phipps, \& Ashcroft, 2015). These results were similar to another study of hospital pharmacists, which found 34 percent of the variance in reporting behavior was explained by two constructs of the TPB (Gavaza et al., 2011).

\section{Theoretical Framework}

TPB is an extension of Fishbein and Ajzen's Theory of Reasoned Action (TRA), and is one of the most widely tested theories of human behavior (I. Ajzen, 1991; Ajzen \& Fishbein, 1980). Ajzen's TPB proposes that behavioral, normative, and control beliefs 
influence an individual's attitude towards the behavior of interest (ATB), subjective norms (SN), and perceived behavioral control (PBC), respectively (see Figure 1). These factors in turn influence the intention (INT) to engage in a behavior, which is described by Ajzen as the immediate antecedent of behavior, and the strongest predictor of actual behavior (I. Ajzen, 1991).Intention is frequently used as a proxy for measuring actual behavior, particularly in instances where direct observation of the behavior of interest is unrealistic or difficult to achieve and the behavior-intention correlation is thought to be high (Ajzen, 2011, p. 1115). Reporting behavior is one of these instances. To obtain a direct measure reporting behavior, researchers would have to accurately identify all errors that occurred (the denominator) against errors reported (numerator). Identifying all errors that occur in health care would be extremely difficult from both a logistical and practical standpoint.

\section{Figure 1}

\section{Theory of Planned Behavior}

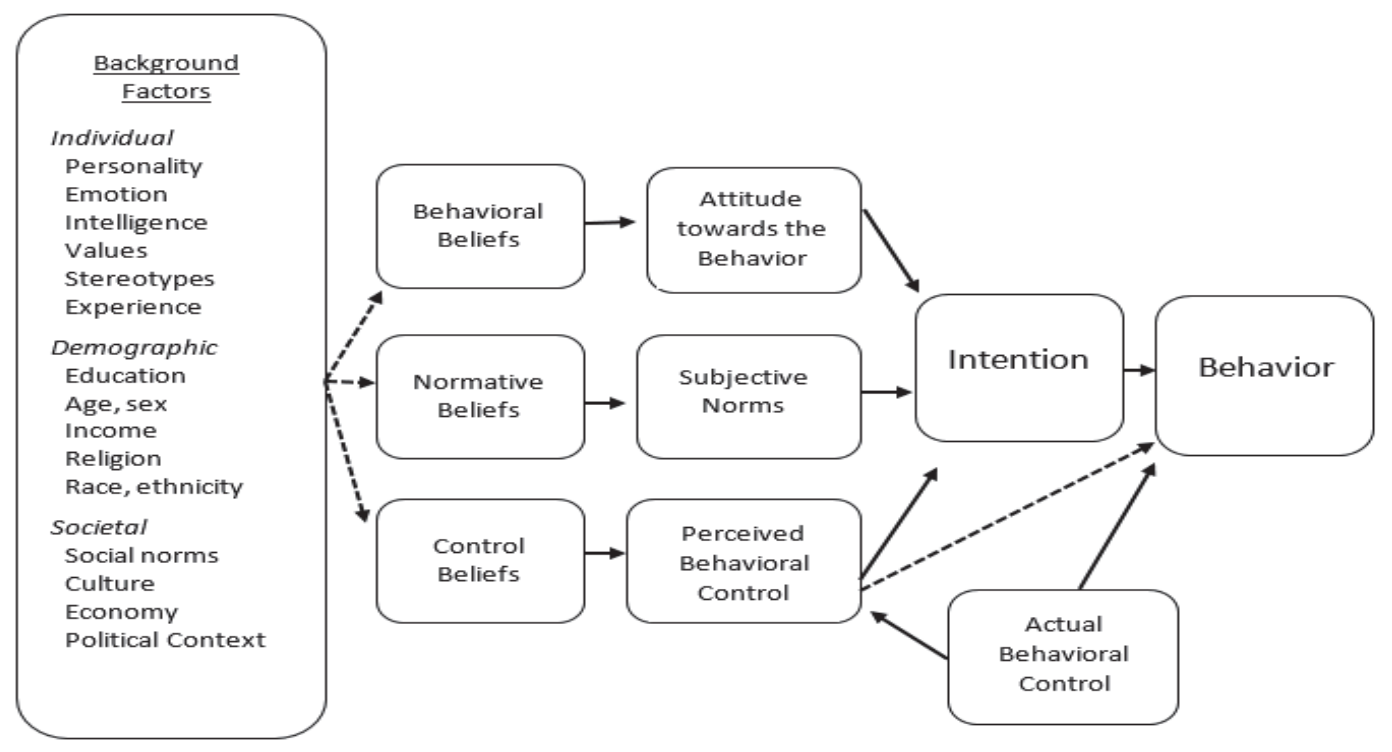


The TPB extends the TRA through the addition of the construct of perceived behavioral control, a construct that captures the degree to which behavior is under volitional control. Volitional control refers to an individual's ability to exercise a degree of control over their behavior, and suggests they have the knowledge, skills, and resources to do so (Frith, 2013). The TRA assumes complete volitional control, whereas the TPB acknowledges that behavioral control is often governed by factors such as adequate time and resources. When a behavior is not under a person's complete control, the variable of perceived behavioral control can directly influence behavior. Although the literature suggests that reporting behavior is under volitional control, there is evidence that the time-consuming process of reporting serves as a substantial barrier (Barach \& Small, 2000; Chiang \& Pepper, 2006; Lee, Yang, \& Chen, 2016; Uribe et al., 2002; Zhao \& Olivera, 2006).

The TPB is a well-validated model that has been applied to numerous public health issues, such as smoking cessation, drug use, HIV testing, condom use, skin cancer protection, exercise, and fat consumption, to explain, examine, and predict human behavior (Appiah, Tenkorang, \& Maticka-Tyndale, 2017; Ayodele, 2017; Hamilton, Kirkpatrick, Rebar, White, \& Hagger, 2017; Lloret Irles, Morell-Gomis, Laguia, \& Moriano, 2017; Record, Harrington, Helme, \& Savage). Since the theory's inception, hundreds of studies have confirmed both its structure and predictive validity. A metareview of over 200 data sets found the TPB predicted, on average, 19 to 40 percent of the variance in intention to engage in a behavior (Rivis \& Sheeran, 2003). Although these effect sizes are impressive, a substantial proportion of variance in behavior remains 
unexplained. Some of this has been attributed to methodological factors, while others may be conceptual.

There is a growing movement towards an expanded version of the TPB to improve model statistics (see Figure 2). One area in which the theory may need expanding is regarding the construct of subjective norms ( $\mathrm{SN})$, which has been shown in some cases to be comparatively weak in its relationship with intention. In the original TPB model, SN are injunctive in nature. Injunctive norms capture what others ought to do, compared to descriptive norms, which reflect what others actually do. There is strong evidence to support adding descriptive norms (DN) into the TPB as an additional predictor variable (Rivis \& Sheeran, 2003). In addition to the medium-to-strong average correlation between DN and intention, DNs have been shown to add an additional five percent to the explained variance in intention, above and beyond attitude, SN, and perceived behavioral control (Rivis \& Sheeran, 2003).

The literature has demonstrated a clear link between psychological safety and reporting behavior, yet there have been very few studies that have attempted to measure the increased utility of including it in a conceptual framework. A study by Lee et al. (2016) utilized an expanded model of the TPB to compare incident-reporting behavior among nursing staff for errors they observed versus errors in which they were personally involved. In both scenarios, psychological safety had a significant effect on the intention to report, with models explaining between 10 and 20 percent of the variance in behavior (Lee et al., 2016). Another study integrating the TPB and psychological safety (safety climate) in military maintenance personnel found the integrated model accounted for 
nearly 50 percent of the variance in the intention to report unsafe behavior (Fogarty \&

Shaw, 2010).

\section{Figure 2}

Theory of Planned Behavior, Expanded Model

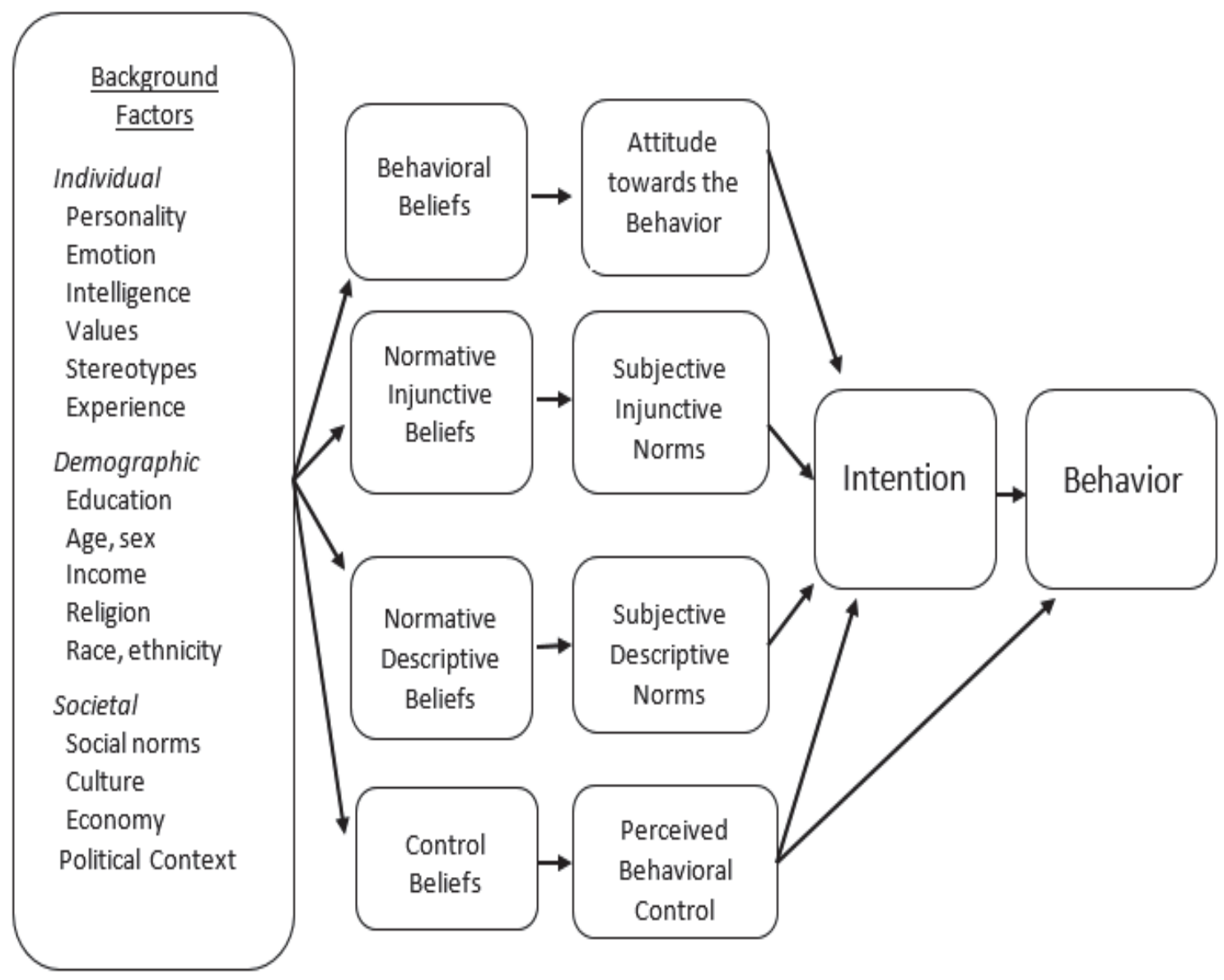

The available data on the error reporting behavior of health care professionals are somewhat partial to nursing and pharmacy staff, with limited information available on physicians. Nurses are at the frontline of the patient safety movement, with the majority of all error reports (80 percent) being made by nursing staff (Tuttle, Holloway, Baird, 
Sheehan, \& Skelton, 2004). Physicians were noted to have made only one percent of all error reports (Rowin et al., 2008). Another study showed physicians only reported serious errors, such as those resulting in death, while nursing staff were more likely to report all events, particularly those causing minimal or no harm (Rowin et al., 2008). This is highly problematic, given that physicians are frontline workers and status hierarchies are a known barrier to reporting (Tucker, Singer, Hayes, \& Falwell, 2008).

The scarcity of data on physician reporting behavior may be handicapping patient safety efforts and indirectly contributing to the belief that reporting is primarily the responsibility of nursing staff (Cook et al., 2004). A brief review of the literature on physician response rates to research surveys was conducted to optimize study recruitment

efforts. A study examining reasons for not participating in postal questionnaire surveys in a sample of general practitioners found the importance of topic and/or relevancy to the practitioner's specialty was very important when deciding to respond (Kaner, Haighton, $\&$ McAvoy, 1998). Other studies suggested multiple follow up reminders and the use of "noncommittal" incentives (i.e. coffee shop gift card) to improve response rates (Abdulaziz et al., 2015; Hocking, Lim, Read, \& Hellard, 2006; Robertson, Walkom, \& McGettigan, 2005).

\section{Contributions of the Current Study}

Error reporting can be conceptualized as a complex health behavior. Engaging health care professionals in this behavior is needed before meaningful impacts on health outcomes can be actualized. However, implementing this behavior change has proved difficult. This study aimed to increase understanding of the predictors of reporting 
behavior using a survey modeled after the theory of planned behavior. The survey assessed the predictive utility of expanding the theoretical model to include a measure of psychological safety and used responses to case study scenarios to capture a novel measure of intention, termed intention simulation. Understanding the strength in which certain variables influence behavior is critical to developing targeted interventions. This is particularly important in the fast-paced, high-stakes industry of health care.

This study extends the literature on reporting behavior through a targeted campaign directed towards physicians. There is disparate information available on physicians, compared to nursing and pharmacy staff. Filling these gaps allows for a full conceptualization of the problem. The airline industry did not transform their safety record by ignoring the role pilots played in the problem and, ultimately, the solution. 


\section{CHAPTER III}

\section{METHODOLOGY}

A theory-driven approach was used to better understand medical error reporting behavior in healthcare professionals licensed in the state of Kentucky. Questions modeled after the theory of planned behavior (TPB) assessed the degree to which an individual was ready to engage in the behavior of error reporting (i.e. the intention to report) and the immediate antecedents of behavior (subjective norms, perceived behavioral control, attitude). The methods described in this chapter were used to answer the following research questions and corresponding study hypotheses:

- Research Question 1(a) - What is the relationship between attitude towards behavior, subjective norms, perceived behavioral control and the intention to report medical errors? Hypothesis 1: The theory of planned behavior will significantly predict the intention to report medical errors.

- Research Question 1(b) - Does the inclusion of additional variables in an expanded theory of planned behavior model explain additional variance in intention scores? Hypothesis 2: Inclusion of background variables, past reporting behavior, and actual behavioral control will significantly predict intention scores. 
- Research Question 2(a) - What is the relationship between psychological safety and the intention to report? Hypothesis 1: Psychological safety will be a significant predictor of the intention to report medical errors.

- Research Question 2(b) - What is the relationship between TPB antecedents of behavior, psychological safety, and the intention to report medical errors? Hypothesis 2: The inclusion of psychological safety as an additional predictor variable in the theory of planned behavior will significantly improve explanatory power of the model.

- Research Question 2(c) - Does the inclusion of psychological safety in an expanded model explain additional variance in intention scores? Hypothesis 3: Inclusion psychological safety as an additional predictor variable in an expanded model of the TPB will significantly predict intention scores.

- Research Question 3 - How does psychological safety influence the intention to report? Hypothesis 1: Psychological safety will independently influence the intention to report.

- Research Question 4 - Can an alternative measure of intention better approximate actual behavior? Hypothesis 1: Intention simulation will serve as a better proxy for actual behavior than generalized intention in predicting error reporting behavior.

\section{$\underline{\text { Participants }}$}

Participants in this study were hospital-based health care professionals licensed to 
practice in the state of Kentucky and included nurses (Registered Nurses, Nurse Practitioners, Licensed Practical Nurses) and physicians (Attending/Staff Physician, Resident Physicians/Fellows, Physician Assistants). Corresponding job titles mirrored categories on the Hospital Survey on Patient Safety Culture released by the Agency for Healthcare Research and Quality (AHRQ, 2004, Section H, Question 4).

\section{$\underline{\text { Recruitment }}$}

A national healthcare marketing company was contracted to conduct an email recruitment campaign for this study. DMD Marketing (DMD) corporation is a fee-forservice organization with access to over 6 million "opted-in" email addresses of US healthcare professionals. DMD has deployed over 300 million emails across 50,000 email campaigns, primarily servicing the pharmaceutical industry (DMD Marketing Corporation, 2018). DMD advertises their physician database as one of the most comprehensive, with over $90 \%$ representation. A certification of authentication provided by media-auditing organization, BPA Worldwide, was available upon request as a guarantee that each email address belonged to a qualified recipient. Email address were a mix of personal and professional address, described by DMD as the practitioner's preferred email, and is described in more detail in the product and services manual (DMD, 2018).

DMD owns proprietary rights and offers access to three healthcare provider databases (physicians, nurses and practitioners) (DMD Marketing Corporation, 2018). Optimization of this email campaign was achieved through audience segmentation, 
including filters which selected only hospital-staffed healthcare professionals (physician, practitioner, nurse) who were licensed to practice in Kentucky. An additional filter in the physician database included all AMA self-designated practice specialty (SDPS) codes. The practitioner and nursing groups were filtered by position, specialty, and education. Filtering for the nursing group included the following positions: advanced practice, licensed practical nurse (LPN), manager, case manager, staff nurse, registered nurse (RN), clinical specialist, and Certified Registered Nurse Anesthetists (CRNA). Executive-level management, administrators, education and staff development, occupational and physical therapists, allied health professionals, school nurses, and contractors were excluded. All education levels were included (associate degree/diploma, BS/BSN, MS/MSN, Doctorate / $\mathrm{PhD}$ ). The practitioner group was also filtered by specialty and included all available specialties, except those specifically referencing outpatient care or education. A one-time data use agreement for the AMA Physician Professional Data (AMA-PPD) was executed, and all components of the email campaign complied with the CAN-SPAM Act of 2003.

DMD marketing corporation was under contract to deploy the survey to 3,000 email addresses randomly selected from each of the three practitioner databases. The physician assistant/nurse practitioner database did not have 3,000 email addresses available for sampling after optimization filters were applied, resulting in a total deployment less nine thousand. 


\section{Data Cleaning}

Data cleaning was conducted to identify and correct errors that could have a negative impact on study results. Following good clinical practices, all data handling procedures were standardized and documented (Van den Broeck, Cunningham, Eeckels, \& Herbst, 2005). Upon closure of the survey, 113 records were exported from Qualtrics into excel. All variables were independently inspected for missing data or unusual responses. Screening including identifying inconsistencies, odd patterns and other unexpected result. This included data values falling outside the expected range. Study variables were renamed and the datafile imported in SPSS.

SPSS variables were recoded from categorical into scale and summary composite scores were calculated. Missing data received a user-defined missing value (-99) to allow SPSS to perform computations by omitting missing values through pairwise or listwise deletion. Double data entry and random spot checks were used as quality assurance measures. All data coding procedures described above were replicated resulting in a seconds or analysis dataset. A 10\% random spot check of responses was conducted for accuracy checks (Van den Broeck et al., 2005).

\section{Data Analysis Plan}

Multiple regression was used to answer the research questions in this study. Ajzen (1991) states that multiple regression, structural equation modeling, latent class analysis are all acceptable methods to determine the importance of beliefs. The assumptions of regression were evaluated prior to data analysis. Scatterplots were used to check for a linear relationship between variables. The assumption of the independence of 
observations was assessed using the Durbin-Watson statistic. A Durbin-Watson value (d) between the critical values of $1.5<\mathrm{d}<2.5$ assumed no first order linear autocorrelation (Field, 2009). Responses from participants were only be measured at one-time point to ensure independence of observations. The presence of homoscedasticity in the data (i.e., equal variance between variables) was assessed by plotting the studentized residuals against the unstandardized predicted values. Levene's test assessed of the equality of error variance.

To determine if multicollinearity (i.e., two or more highly correlated variables) was present in the data, correlation and Tolerance/VIF values were examined. A minimum tolerance value of .10 recommended by Tabachnik \& Fidell (2001) was used to assess this assumption. Tolerance values as high as .25 were found in the literature (Huber \& Stephens, 1993). A maximum Variance Inflation Factor (VIF) of 10 was used to measures the impact of collinearity among variables (Hair, Anderson, Tatham, \& Black, 1995; Kennedy, 1992), although lower maximum values (4 -5) can also be found in the literature (Rogerson, 2001; Pan \& Jackson, 2008). Leverage was used to assess outliers or undue influence among the predictor variables using Cook's distance (D), with a cutoff value of 1 (Cook, 1977). Values greater than one were considered influential and suspicious of being an outlier. Residuals were evaluated for normal distribution through a histogram and a normal probability (P-P) plot and a normal quantile-quantile (Q-Q) plot.

Model summary statistics determined how well the regression model fit the data. The multiple correlation coefficient $(\mathrm{R})$ indicated the level of prediction. The coefficient of determination $\left(\mathrm{R}^{2}\right)$ showed the proportion of variance in the criterion variable 
explained by the predictor variables (i.e., the proportion of variance accounted for by the regression model). The F-ratio tested if the overall regression model was a good fit for the data. Estimated model coefficients were used to determine the how the criterion variable varies with each predictor variable when all other predictor variables were held constant. T values were used to evaluate the significance of each predictor variable, with $p<.05$ indicating significance (Miller, 2009). The PROCESS procedure created and described by Hayes in An Introduction to Mediation, Moderation, and Conditional Process Analysis (2017) was followed to test for a mediating relationship between psychological safety, the antecedents of behavior, and intention.

\section{Dependent Variable}

This study examined the general behavior of reporting medical errors, defined in terms of target, action, context, and time (TACT) as described by Francis et al. (2004, p. 8). Given the difficulties associated with direct observation of this behavior, a measure of intention was used as a proxy to actual behavior. Following TACT methodology, the behavior of interest in this study was defined as follows: target (medical errors), action (reporting intention), context (hospital work area/unit), and time (implicitly, as soon as possible).

To ensure the principle of compatibility was observed, all constructs were measured at the same level of specificity when appropriate (Fishbein \& Ajzen, 1975; 1977; Ajzen, 1988). The principle of compatibility posits that predictive power is increased when measures of the predictor and criterion variables match on the target, 
action, context, and time. Observing this principle minimizes discrepancies between attitude/intention and behavior. A meta-analysis conducted by Kraus (1995) found attitude-behavior correlations increased from $r=.14$ to $r=.50$ when the principle of compatibility was maintained.

\section{$\underline{\text { Sample Size Calculations }}$}

Sample size calculations for this exploratory study were calculated using an $A$ Priori G*Power analysis (F tests; Linear multiple regression: Fixed model, R2 increase) which suggested a sample size of $\mathrm{N}=85$ (Faul, Erdfelder, Lang, \& Buchner, 2007). $\mathrm{G}^{*}$ Power calculations were based on $\alpha=.05$, power $(1-\beta)=.80$, a medium effect size $=$ .30 , and a total of four predictors (attitude, perceived behavioral control, subjective norms, psychological safety). These calculations were consistent with recommendations from Francis et al. (2004), which suggested a sample size of $\mathrm{N}=80$ for a regression analysis with an expected medium effect size of $d=.30$ (Cohen, 1988).

\section{$\underline{\text { Apparatus }}$}

Study data were collected using the web-based survey tool Qualtrics $\mathrm{XM}^{\circledR}$, hosted at the University of Louisville (Qualtrics, 2017). The survey instrument was structured into two sections. The first section consisted of 37 multiple-choice questions, while the second contained six case study scenarios with a maximum of three additional questions. Additional questions were displayed using branching logic and based on item response, with a maximum of 18 possible options. The maximum number of questions for the survey was 55 (range 43-55). The survey included an optional open-ended response box 
for participants to give feedback on the case studies or error reporting in general. The study utilized an unsigned consent form (i.e., preamble), and submission of the survey implied informed consent. Data were stored in an encrypted, password-protected computer in a locked office. Only the investigator and regulatory/oversight officials at the University had access to the data. The institutional review board (IRB) approved the study and all materials (see Appendix A).

\section{MEASURES}

The first section of the survey instrument included four measures corresponding to the constructs of the theory of planned behavior: attitude, subjective norms, perceived behavioral control, and intention [to report medical errors]. Measures of psychological safety and past reporting behavior were also collected. The second section of the survey consisted of six patient safety case study scenarios. Responses to these case studies were used to calculate a measure of intention simulation. The complete survey instrument can be found in Appendix B.

\section{Background Variables}

Verbiage for demographics, past reporting behavior, and safety culture questions was taken directly from the Agency of Healthcare Research and Quality's (AHRQ) Hospital Survey on Patient Safety (SOPS) (AHRQ, July 2013). The SOPS was developed for public use and available free of charge. The instrument was designed to be administered to health care professionals at various levels (individual, unit, departmental, 
and organizational), with good psychometric properties based on data from 50,513 respondents across 331 hospitals in the United States (Sorra et al., 2016). Sorra et al.'s (2016) analysis examined item factor loading, interclass correlations (ICCs), design effects, internal consistency reliabilities, and multilevel confirmatory factor analyses (MCFA). Item factor loadings averaged .80 , with criterion values $>.40$. ICCs averaged .10 , with values between .05 and 5.0 percent being acceptable. Average design effects were 3.10 , with criterion values $>2.0$. Internal consistency reliability values ranged from .62 to .85 with all but one composite with values .70 or greater. The MCFA values at both the unit (.54 -1.0) and individual level (.36 - .93) were within acceptable ranges.

\section{Past Reporting Behavior}

A single statement taken from the AHRQ's Hospital Survey on Patient Safety

Culture (2004, Section G, pg. 4) assessed the number of events reported over the past 12 months, which served as a measure of past reporting behavior. Participants were asked "In the past 12 months, how many medical errors or events [that you personally committed or observed] have you reported to an incident reporting system or a manager/supervisor?" with six response categories ranging from no reports to 21 or more reports. Following scoring methodology used by the AHRQ in their 2016 Comparative Database Report, the number of event reports reported were recoded into binary categories, no reports (0) versus one or greater reports (1), described as percent positive scoring (Chart 5-4, p. 27).

Participants were also asked to report the number of errors they either committed 
or observed over the same 12-month period, using a modified version of the AHRQ's past reporting behavior question (Section G, pg.4). Participants were asked "In the past 12 months, how many medical errors or 'events' have you committed or observed?" with the same six response categories.

\section{Safety Culture}

The Study Group on Human Factors defines safety culture as a collection of “individual and group values, attitudes, perceptions, competencies, and patterns of behavior that determine the commitment to, and the style and proficiency of, an organization's health and safety management" (Sorra et al., 2016, p. 1). The AHRQ's Hospital Survey on Patient Safety Culture contains a total of twelve composites to measure these domains. To keep response burden low, this study included only one composite measure (Communication Openness) and an overall patient safety grade.

\section{Communication Openness}

This composite defined the extent to which staff feel safe to speak up or question authority when issues of patient safety are at stake. This composite was assessed by three items measured on a five-point scale. Response options included: Never, Rarely, Sometimes, Most of the time, Always. Items assessed were: (1) "Staff will freely speak up if they see something that may negatively affect patient care" (2) "Staff feel free to question the decisions or actions of those with more status or authority" (3) "Staff are afraid to ask questions when something does not seem right" (negatively worded). The frequency of responses for each survey item was calculated and negatively worded items 
were reverse coded following the methodology outlined in the AHRQ Hospital Survey on Patient Safety Culture: User's Guide to calculate composite and percent positive scores (Sorra et al., 2016, p. 30).

\section{Patient Safety Grade}

This question allows participants to assign an overall "grade" on patient safety to individual work units assessed by one item: "Please give your work area/unit in this hospital an overall grade on patient safety based on the past 12 months" with five response categories: Excellent (A), Very Good (B), Acceptable (C), Poor (D), and Failing (F).

\section{Psychological Safety}

Psychological safety is defined as a "shared belief that a team is safe for interpersonal risk taking" (Edmondson, 1999). Psychological safety was measured using Edmondson's (1999) Psychological Safety Scale, which contain seven questions assessed on a five-point scale from "Strongly Disagree" to "Strongly Agree." Participants were asked to answer questions based on their work/team environment. Items were: (1) "If you make a mistake on this team, it is often held against you", (2) "Members of this team are able to bring up problems and tough issues", (3) "People on this team sometimes reject others for being different", (4) "It is safe to take a risk on this team", (5) "It is difficult to ask other members of this team for help", (6) "No one on this team would deliberately act in a way that undermines my efforts", and (7) "My unique skills and talents are valued and utilized while working with members of this team". The mean of the seven psychological safety items were calculated to give an overall score of psychological 
safety. Higher scores were reflective of higher levels of psychological safety.

\section{Theory of Planned Behavior}

The primary measure used in this study was based on the Theory of Planned Behavior and constructed following the methodology outlined in the Manual for Health Services Researchers (Francis et al., 2004). Guidance from Fishbein \& Ajzen's TPB Questionnaire Construction was also consulted (Fishbein \& Ajzen, 2010). The domains of attitude towards behavior, subjective norms, perceived behavioral control, and intention were assessed on a five-point scale and are described below.

\section{Attitude Towards Behavior}

Ajzen describes attitude towards a behavior as a person's positive or negative view of performing the behavior in question" (1991). A direct measurement of attitude towards the behavior was assessed by a single stem statement followed by four 5-point adjectives. The stem statement was: "Overall, I think reporting medical errors is:" Response items were: 1) "Good/Bad Practice," which captures the overall evaluation of the attitude towards a behavior, 2) "Harmful/Beneficial," 3) "Pleasant/Unpleasant," 4) "Worthless/Useful." Items were arranged to reflect a mix of negative and positive endpoints. The mean of the four attitude item scores were calculated to give an overall attitude score. Items with negative endpoints towards the right "Good/Bad; Pleasant/Unpleasant" were recoded so that higher numbers reflected a positive attitude towards the behavior. An estimate of internal consistency was computed (Cronbach's alpha) for all measures. 


\section{Subjective Norms}

Subjective norms $(\mathrm{SN})$ reflect an individual's perception of the social pressure to engage or not engage in a behavior (Ajzen, 1991). There are two types of normative behaviors, described as injunctive and descriptive norms. Injunctive norms refer to perceptions of behavior that is approved or disapproved by others (i.e., what others ought to do), while descriptive norms refer to how others actually behave (Cialdini, Reno, \& Kallgren, 1990). This study assessed both types of normative behavior through four questions measured on a five-point scale. Three items assessed injunctive subjective norms, (a) "It is expected of me that I report medical errors;" (b) "I feel under social pressure to report medical errors;" and (c) "Most people who are important to me thought that (I should/I should not) report medical errors." The fourth question, (d) "Most people who are important to me report medical errors," captured descriptive subjective norms. Items with negative endpoints towards the right were recoded so that higher numbers reflect greater social pressure to engage in the behavior. The mean of the three subjective norm (injunctive) items were calculated to give an overall subjective norm (SNI) score. All four questions were combined to give an overall subjective norm average score.

\section{Perceived Behavioral Control}

Perceived behavioral control (PBC) reflects an individual's confidence in performing the target behavior (Ajzen, 2006). This encompasses a “person's self-efficacy and beliefs about the controllability of the behavior" (p. 21). A direct measurement of 
PBC was assessed by four questions, utilizing a five-point scale. Items were arranged to reflect a mix of negative and positive endpoints. The four items that assessed $\mathrm{PBC}$ were (a) "I am confident that I could report a medical error if I wanted to;" (b) "For me to report a medical error is;" (c) "The decision to report a medical error is beyond my control;" and (d) "Whether I report a medical error or not is entirely up to me." The mean of the four PBC item scores were calculated to give an overall PBC score. Items with negative endpoints towards the right "Easy/Difficult" were recoded so that higher numbers "reflect a greater level of control over the target behavior" (Francis et al., 2004, p. 22).

$\underline{\text { Intention }}$

The intention to report medical errors was used as a proximal measure of behavior in the absence of actual behavior (Ajzen, 2006). Intention (generalized) was measured by three questions: (a) "I expect that I will report medical errors;" (b) "I want to report medical errors;" (c) "I intend to report medical errors." Questions were scored using a five-point scale ranging from "Strongly agree" to "Strongly disagree."

\section{CASE STUDIES}

Responses to case studies were used in the second section of this study to calculate a measure of intention simulation. Case Studies (i.e., case vignettes, case studies, case reports) are used in both qualitative and quantitative research as a nonthreatening method to explore judgments and actions of sensitive topics. This study used 
the Constant Variable Value Vignette method (Kathiresan \& Patro, 2013), a variant of the case study method, to assess differences in reporting behavior across different types of health care providers and different situations (Barter \& Renold, 1999). Originating in business ethics research, this method presents all participants with identical vignettes and focuses solely on outcomes. Strengths associated with using vignettes include a quick and cost-effective method of obtaining data on topics that might otherwise be difficult to obtain. However, the limitations associated with using vignettes include threats to external validity. Parkinson and Manstead (1993) argue against their use, suggesting that vignette data does not allow for generalizations and can only be understood within the context of the vignettes. Other reports propose vignettes may overestimate actual behavior (Eifler, 2007).

Cook and Hoas (2004) developed the case studies that were used in this study as part of their Advancing Patient Safety study supported by the AHRQ (AHRQ R01HS11930). Case studies were taken from University of Montana's Health Care Ethics Study website (Cook \& Hoas, 2020). This website resource contains 53 patient safety case studies based on actual events encountered by rural health care providers who participated in the Healthcare Ethics Study over the course of four years, in 30 hospitals, across nine states. Nineteen of the 53 case studies include a full summary analysis developed in conjunction with researchers at Rush Medical College. The analysis model used for summarizing each case includes defining the topic, issues, learning points, guides, disclosure, and room for improvement, as well as several background questions. Case studies included both clinical and ethical issues, with some cases containing more 
than one type of IOM classified error (i.e., diagnostic, treatment, preventive, other). All materials in the patient safety manual (including case studies) were available free of charge. No copyright permissions were required for the use of these materials.

\section{Case Study Selection Process}

Case studies considered for inclusion in this study were limited to the 19 summarized cases. Cases with an exclusive focus on child/infant health issues $(n=5)$ were excluded, and multiple cases related to medication and dosing errors were omitted to minimize redundancy of error types $(n=2)$. Cases without clear-cut error classification such as polypharmacy $(n=1)$, those with a strong ethical focus $(n=1)$, and complex case scenarios $(n=1)$ were also excluded. To minimize respondent burden, cases studies with a word count $>350(n=4)$ were also excluded. Consideration was taken to have representation of each type of error (diagnostic, treatment, prevention). Further selection criteria included the level of harm caused by the error in the scenario. To assess if responses were related to the degree of harm, cases from each group (death, permanent harm, and no permanent harm/near miss) were selected. An adverse event resulting in death or permanent harm is subject to mandatory reporting requirements, whereas reporting events that inflict little to no permanent harm are voluntary. 


\section{Table 6}

Case Studies by Error Type and Issue

\section{Case \#1 Treatment - Error in dose of drug}

Case \#2 Prevention - Inadequate monitoring or follow up treatment

Case \#3 Treatment - Error in the dose of drug

*Permanent Harm

Case \#4 Diagnostic - Failure to employ indicated test resulting in death

*Sentinel Event

Case \#5 Treatment - Error in the performance of an operation, procedure or test - Avoidable delay in treatment

Case \#6 Treatment - Error in administering the treatment - Error in the dose or method of using a drug

*Sentinel event is defined by the Joint Commission as a patient safety event resulting in death, permanent harm, or severe temporary harm and intervention required to sustain life

\section{Error Recognition}

All cases presented in this study contained reportable error scenarios. An error recognition score was calculated by first asking participants if they believed an error had occurred. Yes, answers were summed and represented the participants raw error recognition score. Score were converted into percentages for classification purposes. Responses of 'unsure, maybe, or no' were combined and counted as a negative endorsement or lack of recognition. A total of 72 participants (64 percent) completed all 6 cases. Average recognitions score were out of a maximum of $\operatorname{six}, \mathrm{M}=4.94, \mathrm{SD}=1.05$. 
The average recognition score was 82 percent. Over $37 \%$ of the sample correctly identified all six cases as containing an error (i.e. 100\%), while $31 \%$ received a failing score (67 percent or less).

Recognition scores were examined by position (physician, practitioner, nurse) to assess for any hierarchical or positional differences. One-way ANOVA found no significant differences across the groups $(\mathrm{p}=.971)$. Nurses had the highest percent correct recognition scores $(M=82.76, S D=19.15)$, followed by physicians $(M=81.75$, $\mathrm{SD}=17.40)$, and practitioners $(\mathrm{M}=81.67, \mathrm{SD}=16.13)$. Differences in percent correct recognition scores related to psychological safety were explored and found to be nonsignificant, $F(2,67)=0.76, p=.47$. Individuals working in environments with high psychological safety had the highest percent correct error recognition score $(\mathrm{M}=88.89$, $\mathrm{SD}=8.33$ ), followed by those working in environments with low psychological safety, who had higher mean error recognition scores $(\mathrm{M}=83.33, \mathrm{~S} .=17.57)$ compared to those in environments with moderate psychological safety $(\mathrm{M}=81.05, \mathrm{SD}=18.86)$.

\section{Intention Simulation}

Intention simulation is the most time-consuming method of measuring intention that may approximate actual behavior better than traditional measure of generalized intention. Intention simulation was measured by captured by asking participants to read each case study and respond to three questions, displayed using branching logic driven by item response. The first question captured error recognition. Participants were asked to decide if an error occurred, with three response categories (Yes, Maybe, No). Individuals 
answering no, an error did not occur, did not receive the additional questions and the survey advanced to the next case study. Individuals answering yes or maybe were presented with two additional questions and were first asked to make a reporting decision, "Would you report this to the appropriate authority, such as an incident reporting system or supervisor/manager?". Responses were assessed on a five-point scale (Definitely Yes/Definitely Not) and served as a measure of intention simulation.

Lastly, participants were asked "Do you think your colleagues would report this case to the appropriate authority, such as an incident reporting system or supervisor/manager?" assessed on the same five-point scale. This question was added to test for the presence of social desirability bias, which is discussed in detail in Chapter V. Responses to this question were compared with individual responses to determine if participants portrayed themselves and their reporting behavior in a more positive manner than their colleagues. Participants were also provided the opportunity to offer any comments, thoughts, or suggestions related to the general topic of reporting medical errors in an open-ended comment box.

\section{Pilot Testing}

Pilot testing was conducted in two phases. The first round of pilot testing was designed to find the optimal target number of case studies to minimize response burden. Four health care professionals (two physicians and two registered nurses) were given nine case studies to review. Responses from the pilot group indicated six cases as the optimal number. All participants preferred the shorter, more concise case studies. A review of the 
literature on studies utilizing case vignettes found ranges from 1-30 vignettes presented to research participants (Livorsi, Comer, Matthias, Perencevich, \& Bair, 2016; Martinez et al., 2014; Schroder, Behnke, Gastmeier, Schwab, \& Geffers, 2015). Given this feedback, plus the need to include cases from each error category and level of impact, six case studies were selected for inclusion in the final survey.

A second round of pilot testing on the entire survey instrument was conducted with a group of ten local health care professionals. Pilot participants were provided a link to the entire survey and asked to provide feedback using six questions recommended by Francis et al., (2004, p. 27). Questions included: (1) “Are any items ambiguous or difficult to answer?", (2) "Does the questionnaire feel too repetitive?", (3) "Does it feel too long?”, (4) “Does it feel too superficial?", (5) “Are there any annoying features of the wording or formatting?", (6) “Are there inconsistent responses that might indicate that changes in response endpoints are problematic for respondents who complete the questionnaire quickly?". Responses from the pilot participants indicated several wording and formatting issues and included some mild concern over the length of the survey, including the use of a seven-point scale. The survey instrument was revised accordingly, including the use of a five-point scale. 


\section{CHAPTER IV}

\section{RESULTS}

This chapter describes the results of the study as executed following the methodology outlined in Chapter III. Results of the statistical analyses corresponding to each of the research questions are presented and a discussion of these findings and their significance to public health follows in Chapter V.

The purpose of this study was to use a theory-driven approach to better understand the factors that predict error reporting behavior in a sample of health care professionals. Using the theory of planned behavior (TBP), this study examined the relationship between the antecedents of behavior (attitude towards behavior, subjective norms, perceived behavioral control) and the intention to report medical errors (a proxy for behavior when observing actual behavior is difficult). Psychological safety, a construct known to influence reporting behavior, was added to the model as an additional predictor variable to increase the explanatory power. Lastly, this study used responses to case study scenarios to capture an alternate measure of intention (intention simulation) to determine if it improved the predictive utility of the model.

\section{$\underline{\text { Survey Deployment }}$}

The survey went live on October 18, 2018, to a total of 7,604 email addresses. Email recipients received an email with a brief introduction to the survey and were 
provided a unique link directing them to the online survey instrument that has housed on a Qualtrics $₫$ cloud server. The email deployment report provided by DMD indicated 7,527 (99 percent) of emails were successfully delivered, and 77 (one percent) were undeliverable (i.e., hard bounces due to bad mailboxes or bad domains). Response results showed 14 percent of delivered emails $(n=1,054)$ were opened and 1.5 percent $(n=113)$ clicked on the survey URL (see Figure 3). The majority of recipients (74 percent) engaged with the survey email via a mobile device and only 26 percent used a desktop platform. The survey took approximately 13 minutes to complete $(M=12.5, S D=9)$. Within seven days of the initial email deployment, DMD sent a second or "echo" email to non-responders.

DMD provided a detailed tracking report two weeks after the second deployment, which summarized the total number of emails that were sent, delivered, opened, and clicked. Overall, this study had a $19.24 \%$ open rate, meaning that nearly $20 \%$ of all emails were opened upon delivery. This rate was described as a "fabulous" success by the DMD project management team. Further statistics reveal that $13.41 \%$ clicked on the survey link that was embedded in the survey email, constituting a unique click. These statistics were substantially higher than the average unique click rate of $8 \%$ promoted by DMD in their proposal package.

Deployment report statistics suggest the campaign topic was interesting and enticed the recipient to open the email as evidenced by a high open rate. Results further suggest that the majority of participant who opened the email intended to access the survey as shown by a unique click rate only slightly lower than the open rate. Compared 
to industry standards, this email marketing campaign was considered highly successful. Benchmark data accepts measures of campaign success as open rates between $15 \%-25 \%$ (Campaign Monitor, 2020). Despite a high open rate, the final response rate of this study was less than 2 percent, suggesting a significant response bias. This significantly lower than expected yield of research responses could have been related to survey topic, disinterest, time constraints, or other factors. Future studies should consider a mixed methods approach or using funds to incentivize participants to maximize attrition.

\section{Figure 3}

Study Recruitment Flow Chart

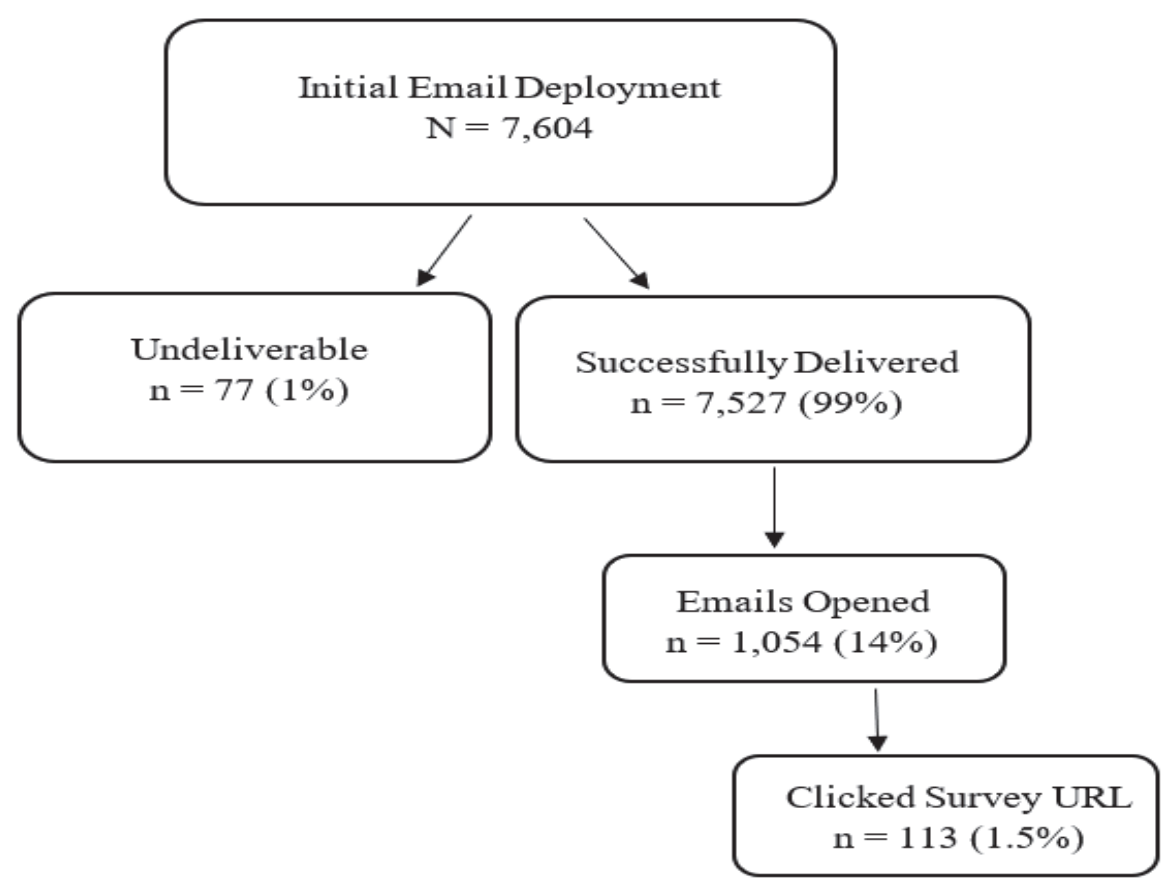




\section{Survey Response}

Qualtrics captured survey progress on 113 health care professionals who clicked on the survey URL. Participants completing $\leq 2$ percent were excluded $(n=7)$, as they provided no usable data. A total of 106 participants had progress completion rates of 11 percent or greater. No participants had survey progress between three and 10 percent. Participants with survey progress $\geq 44$ percent $(n=91)$ were deemed to have useful data, meaning they completed the majority of questions related to the main study questions.

The cut-off value of 44 percent was determined by a frequency analysis, which revealed rates less than 44 percent had a substantial amount of missing data on all measures. Over 64 percent of participants $(n=72)$ completed all survey items.

\section{Figure 4}

Survey Response Rate

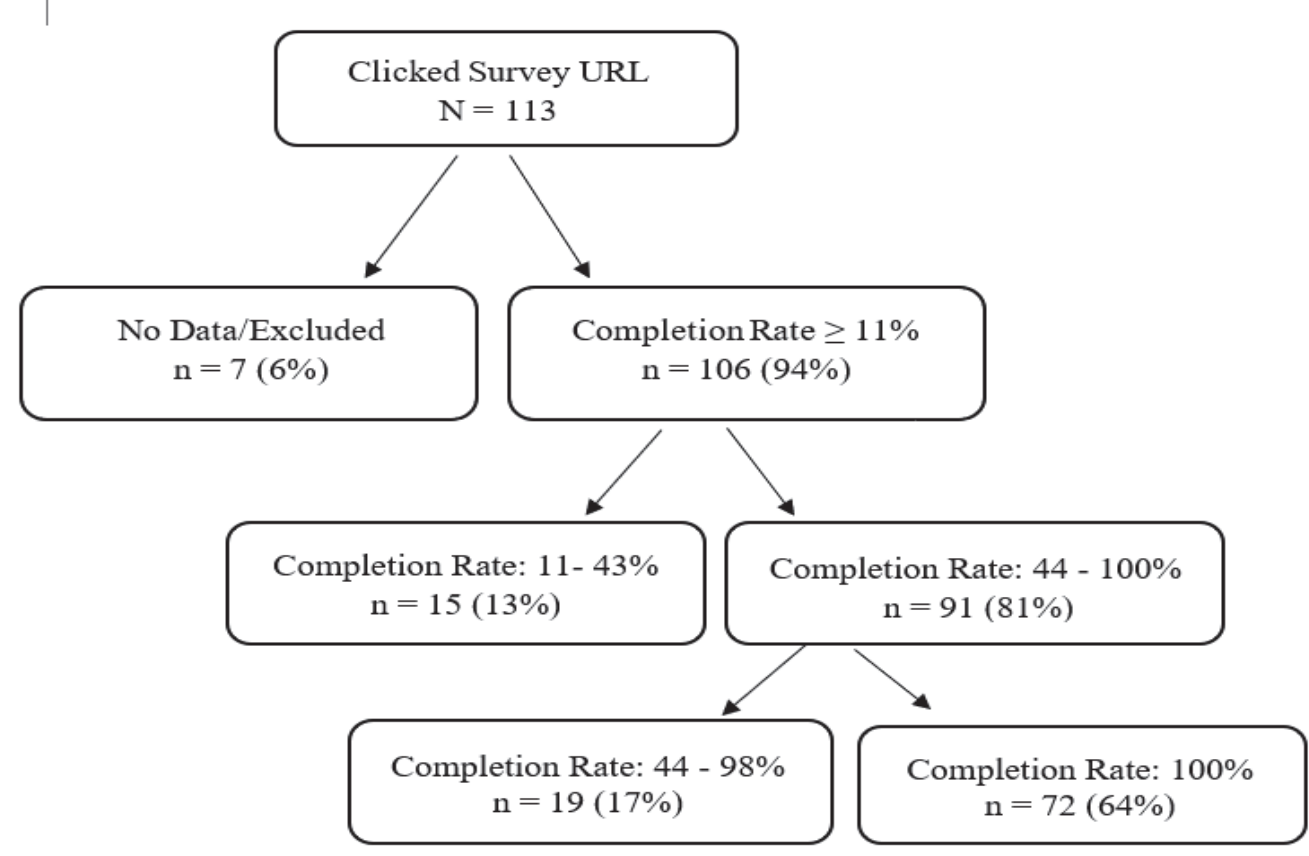




\section{$\underline{\text { Descriptive Statistics }}$}

Initial exploration of the data were conducted on 106 participants using SPSS ${ }^{\circledR}$ version 26 (IBM Corp. SPSS, Released 2018). Frequency statistics included mean, standard deviation, and range (see Table 1). Participants included 30 male and 76 females, aged 23 to 77 years (male: $M=55.6, S D=11.6$; female: $M=51.3, S D=12.2$ ). The average age of participants was 52.5 years $(\mathrm{SD}=12.1)$. The majority (96 percent) described themselves as white, with four percent identifying as any other race. Sample characteristics of participants completing $44-100 \%$ of survey responses $(n=91)$ items were computed and compared to full sample statistics $(n=106)$. Results did not significantly differ with 26 male and 65 female, average age $M=52.2$ years (male: $M=$ 55.0, $S D=12.2$; female: $M=51.5, S D=12.1)$

All participants were hospital staffed employees based on predetermined survey recruitment filters. Registered nurses (RNs) were the most represented staff position (40 percent), followed by attending/staff physicians (30 percent), nurse practitioners (23 percent), and physician assistants (7 percent). No Licensed Professional Nurses (LPNs) completed the survey, and only one medical resident attempted the survey. This individual completed less than 2 percent of items and was excluded from any analyses.

Nearly half of the sample (47 percent) reported working in their profession for 21 years or more, compared to 11 percent who had five years or less experience. The majority of participants (29 percent) had worked at their current hospital or facility between one and five years. Primary work area/unit was assessed by asking participants to identify the work area, department, or clinical area of the hospital where they spend 
most of their work time or provide the majority of their clinical services. Medicine (nonsurgical) was the most reported work area, with 37 percent of respondents identified as belonging to this quasi catch-all category. The next highest area represented was obstetrics (19 percent), followed by surgery (10 percent). The least reported areas were the categories of many different units or no specific unit (two percent) and anesthesiology (three percent). Other specialties reported were psychiatry/ mental health (seven percent), emergency department (seven percent), intensive care unit/any type (six percent), pediatrics (seven percent), and rehabilitation (four percent).

Table 1

Demographic Characteristics of Sample

\begin{tabular}{lllll}
\hline Factor & $\begin{array}{l}\text { Sample } \\
\mathbf{N}=\mathbf{1 0 6}\end{array}$ & $\begin{array}{c}\text { Physicians } \\
\mathbf{n = 3 2}\end{array}$ & $\begin{array}{l}\text { Nurses } \\
\mathbf{n = 4 3}\end{array}$ & $\begin{array}{l}\text { Practitioners } \\
\mathbf{n = 3 1}\end{array}$ \\
\hline Age (yrs.) & 52.8 & 53.6 & 49.1 & 56.3
\end{tabular}

Gender (\%)

Male 29

72

7

13

Female

71

28

93

87

Race (\%)

White

96

94

98

97

Other

4

6

2

3 
Less than 1 year

1 to 5 years

6 to 10 years

8

9

6

11 to 15 years

10

13

2

13

16 to 20 years

22

9

10

10

21 years or more

48

13

30

23

56

42

48

\section{Primary Work Area/Specialty}

No specific unit 2

Medicine (non-surgical) 36

Surgery

9

Obstetrics

18

Pediatrics

7

Emergency Department

7

Intensive Care Unit

6

Psychiatry/Mental Health 8

Anesthesiology

3

Rehabilitation

4

3

33

17

7

3

13

3

13

7

-

7

3

$17 \quad 64$

$10 \quad 3$

$38 \quad 3$

4

13

53

12

5

7

2

Years at Hospital (\%)

Less than 1 year

1 to 5 years

6 to 10 years

11 to 15 years

16 to 20 years

21 years or more
10

28

16

15

11

20
13

23

23

10

7

24
10

28

14

17

14

17
7

35

10

14

10

24 


\section{Hierarchy}

There is a well-established hierarchy in the health care industry. To examine the effects of hierarchy, staff positions were ranked and categorized into three hierarchical groups (high, mid, low). Attending/staff physicians were considered to be at the top of the hierarchy (high) and registered nurses at the bottom (low). Based on a lack of consensus in the literature regarding the hierarchical position of physician assistants versus nurse practitioners, both occupations were placed into one category under the general title of "practitioners" and assigned the mid-position in the overall structure.

Frequency analysis revealed a relatively uniform distribution of participants across the three groups with 30 percent $(n=32)$ in the high or physician group, 29 percent $(n=31)$ in the mid or physician assistant/nurse practitioner group, and 41 percent $(n=43)$ in the low or registered nurse group. Correlation and multiple regression were used to evaluate how well the TPB constructs and psychological safety predicted generalized intention scores for each of the three hierarchical groups (physician, practitioner, nurse).

Physician Group: A correlation matrix between the TPB constructs, psychological safety, and intention measures for the physician group found generalized intention scores were significantly associated with attitude towards behavior and subjective norms. Psychological safety was significantly correlated with intention (generalized), attitude, and subjective norms. The only other significant correlation was between intention simulation (i.e. responses from case studies) and attitude scores $(r=$ $.55)$. 
Multiple regression was used to predict generalized intention scores for the physician group. The model with all four predictors (attitude, subjective norms, perceived behavioral control, and psychological safety) produced $\mathrm{R}^{2}=.589, F(4,17)=6.10, \mathrm{p}=$ .003. Psychological safety had a significant positive regression weight, indicating physicians with higher psychological safety scores (after controlling for the other variables in the model) had higher intention scores. This model explained $58.9 \%$ of the variance in intention scores for the physician in this sample.

Practitioner Group: A correlation matrix found perceived behavioral control was significantly correlated with generalized intention, attitude towards behavior and subjective norms. The multiple regression model with all four predictors produced $\mathrm{R}^{2}=$ $.470, F(4,20)=4.44, \mathrm{p}=.010$. This suggests that the model significantly explained $47 \%$ of the variance in intention scores for the practitioner (physician assistants/nurse practitioners) in this sample. Perceived behavioral control had a significant positive regression weight, indicating practitioners with higher perceived behavioral control scores were expected to have higher intention scores.

Nurse Group: A correlation matrix between the TPB constructs and intention measures for the nurse found intention was significantly correlated with all TPB constructs. Psychological safety also had significant correlations with subjective norms and perceived behavioral control. The multiple regression model with all four predictors produced $\mathrm{R}^{2}=.484, F(4,26)=6.09, \mathrm{p}=.001$. Perceived behavioral control and attitude towards behavior had a significant positive regression weights, indicating practitioners with higher perceived behavioral control and attitude scores were expected to have 
stronger intention scores. Overall, this model explained $48.4 \%$ of the variance in intention scores for the nurse group.

\section{$\underline{\text { Risk Categorization }}$}

Participants were asked to identify a work area or unit where they spend the majority of their time administering care. These areas were stratified into risk categories based on Kessler, Sage, and Becker's methodology (Carroll \& Buddenbaum, 2013). High-risk classification was based on malpractice premiums and included five specialties: emergency medicine, obstetrics/gynecology, anesthesiology, radiology, and surgery. All other specialties were categorized as average to low-risk. Based on this classification strategy, 62 percent of participants $(n=64)$ reported working in an 'average to low-risk' specialty, while 38 percent $(n=39)$ in high-risk areas. Participants working in high-risk specialties were more likely to be a registered nurse, specifically working in the field of obstetrics.

\section{$\underline{\text { Past Reporting Behavior }}$}

Past reporting behavior was assessed using a question from the AHRQ Hospital Survey on Patient Safety. Participants were asked how many errors they had reported over the past 12 months. Over half the participants (56 percent) indicated they made no reports. The remaining reported between one and two reports ( 28 percent), three to five reports (11 percent), six to 10 reports (four percent), and one participant (one percent) reported making 21 or more reports.

The number of events were recoded into binary categories based on positive 
responses, no reports (56 percent) versus one or more reports (45 percent). Participants were also asked to report the total number of errors observed or committed over the same 12-month time period. For this question, 44 percent reported observing or committing no errors, one to two errors (34 percent), three to five errors (13 percent), six to 10 errors (three percent), 11 to 20 (three percent), and 21 or more errors committed or observed was reported by the remaining three percent of participants.

\section{$\underline{\text { Reporting Behavior }}$}

A new variable was created using the number of errors reported (numerator) over the total number of errors committed or observed (denominator). This calculation reflected the proportion of time (percent) that an individual reported an error. A total of 92 participants provided valid answers for both questions. Missing or non-valid data (values $>1.0$ ) were observed for $n=21$ responses. Values greater than 1.0 reflected more errors being reported than were observed/committed and were excluded from this analysis. Values equal to 1.0 reflect complete agreement, meaning all errors were reported. This was observed in 83 percent of the sample.

Underreporting, determined by values $<1.0$, was observed in 17 percent of the sample $(n=16)$. Eleven participants within the underreporting subgroup indicated making no error reports. The degree of underreporting varied, with 12 percent of participants failing to report 11 to 20 errors and 13 percent failing to report 21 or more errors. Figure 5 shows a graph of participants responses indicated by the frequency of events (not percent) observed or committed against the number of events reported. Instances where the reported line (blue line) is greater than the observed or committed 
line (red line), represent invalid data and are displayed for illustrative purposes. This unusual finding suggests participants did not understand the question or were inconsistent and perhaps untruthful in answering.

\section{Figure 5}

Reporting Behavior: Errors Committed or Observed and Errors Reported

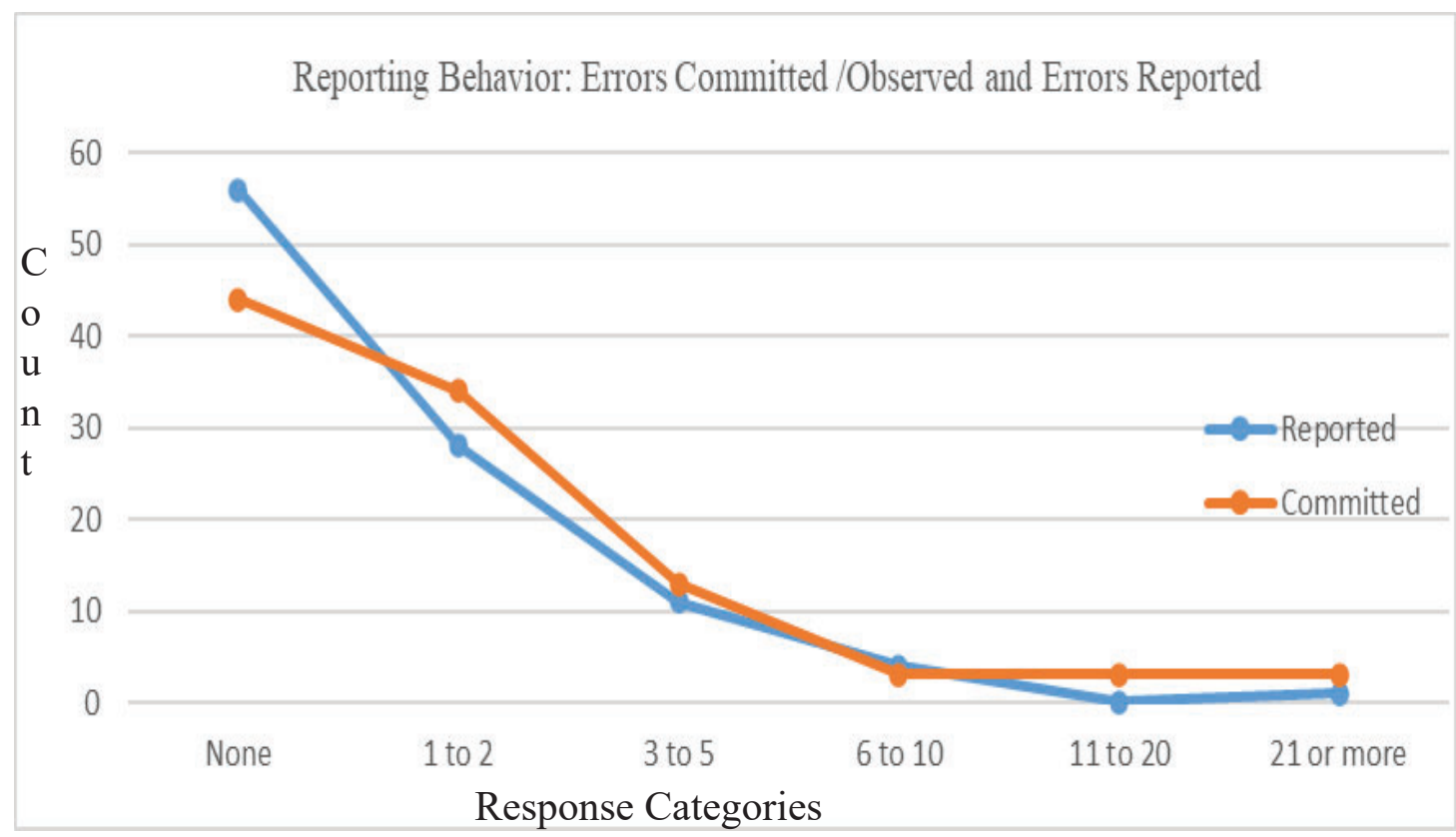

To assess whether reporting behavior significantly differed across staff position (i.e. did nurses report more often and accurately than physicians, for example), a chisquare analysis was conducted. This analysis looked at reporting behavior across the three staff position groups (physicians, practitioners, and nurses) with a null hypothesis that reporting behavior was independent of position and all groups would report equally. Results indicated the assumption of a chi-square analysis had been violated with 33.3 
percent of cells having less than the minimum expected count ( $>20$ percent is considered an assumption violation).

Due to this violation, Fisher's exact test was used which showed a nonsignificant association between reporting behavior and staff position, $p=.357$, suggesting difference between groups were not statistically different. A measure of effect size, Cramer's V, indicated a moderate relationship $(V=.25)$. Looking at the data by position revealed that physicians had the highest rate of underreporting, with 26 percent receiving a reporting scores less than one. Nursing staff followed closely, with an underreporting rate of 20 percent. The practitioner group, which included both nurse practitioners and physician assistants, had the lowest rate of underreporting at three percent.

\section{$\underline{\text { Safety Culture }}$}

Participants were asked to describe the safety culture of their work environment. Patient safety culture was assessed by two measures taken from the AHRQ Hospital Survey on Patient Safety Culture. Communication openness measured the degree to which staff felt at ease to speak up and question authority on issues of patient safety. A descriptive analysis of the data was done and summary scores were converted to standardized values to assess for outliers. Two summary scores had $\mathrm{Z}$ values less than -3 and were considered outliers, using cutoff values slightly less conservative than $+/-3.29$ (Tabachnick \& Fidell, 2007). These scores were treated as missing values and excluded from further analyses. Overall, the sample had a mean communication score of $M=11.36$ $S D=1.83$ out of a maximum score of 15 , and was normally distributed.

An additional measure of safety culture asked participants to grade their 
hospital/facility using a grading scale from A to F (see Figure 6). The majority of hospitals or facilities (88 percent) received a safety rating of excellent (A) or very good (B). The remaining scores ranged from acceptable (C; 9 percent), to poor ( $\mathrm{D} ; 1$ percent), and, lastly 2 percent gave their hospital a failing grade (F). A crosstabulation of safety grade by practitioner group, or hierarchy, found no significant differences between groups. Data management revealed poor to failing safety grades were reported by one nurse practitioner and two registered nurses.

\section{Figure 6}

Hospital Safety Grade

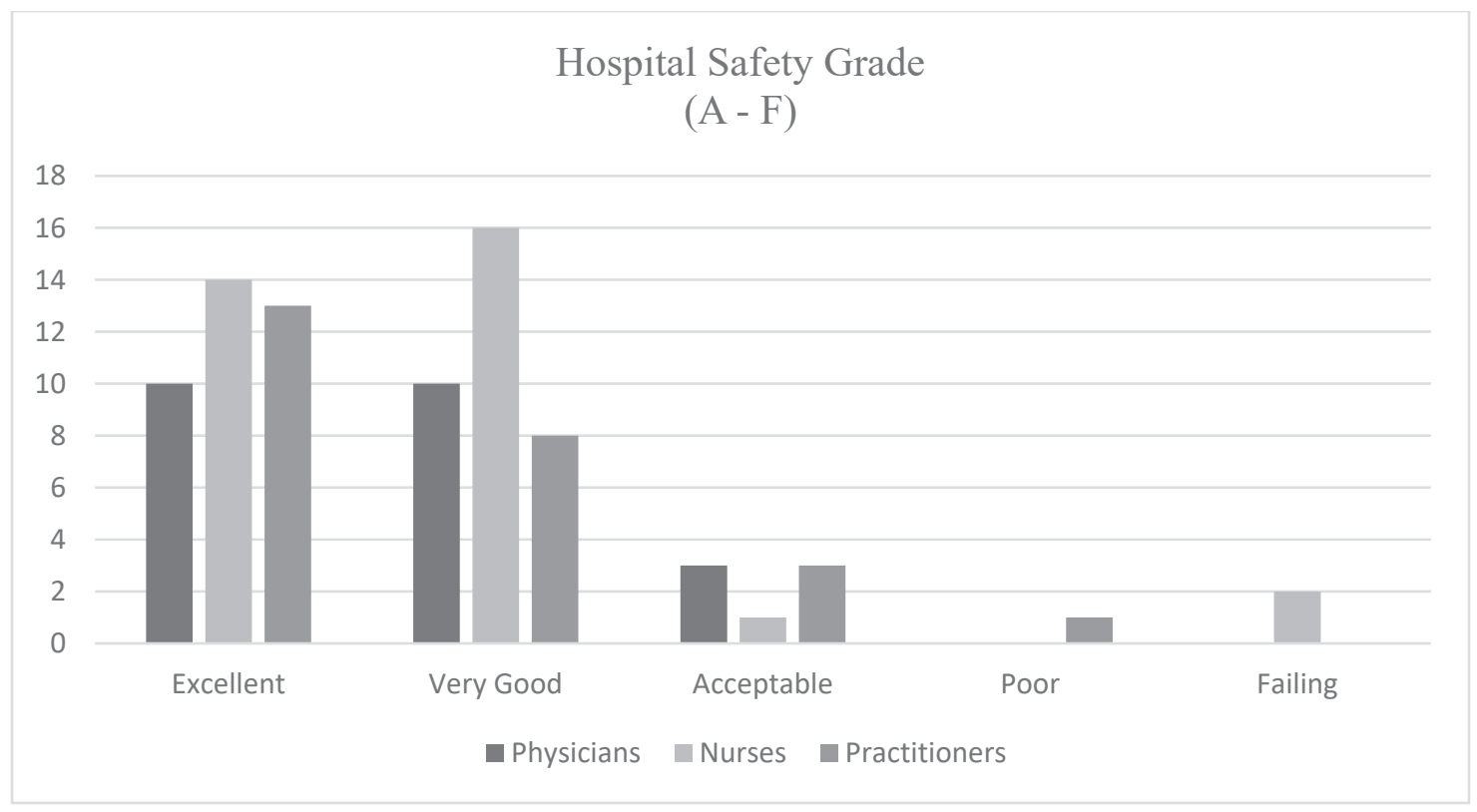

The relationship between reporting behavior and hospital safety grade was explored. There was a significant correlation between underreporting and safety rating, $r(79)=.41, p<.001$. Participants who underreported medical errors gave their hospital or facility lower safety ratings. Poor or failing ratings were provided by eight percent of 
those who underreported, compared to only three percent in the groups who reported 100 percent of errors. Conversely, 91 percent $(n=61)$ of participants who reported 100 percent of errors gave a safety rating of very good to excellent, compared to 67 percent of those who underreported. Safety rating by position was also examined. No significant correlations were found; a crosstabulation analysis revealed that the physician group was less likely to give their hospital or facility a poor to failing rating compared to the other groups.

\section{$\underline{\text { MEASURES }}$}

\section{$\underline{\text { Reliability Analysis }}$}

Cronbach's alpha, an index of internal consistency, was calculated to establish the reliability of each measure (Everitt, 1998). Measures included subscales of the theory of planned behavior (intention, attitude, subjective norms, and perceived behavioral control), and psychological safety. Items were deleted to improve scale consistency where indicated and descriptive statistics are based on finals scales. Intention and attitude subscales of the theory of planned behavior both demonstrated strong internal consistency. Cronbach's alpha for intention was $\alpha=.87$ and $\alpha=.85$ for attitude. Cronbach's alpha for the subjective norm subscale was improved by deleting two questions $(\alpha=.58)$, and perceived behavior control had the lowest measure of internal consistency $(\alpha=.44)$. Cronbach's alpha for the psychological safety scale $(\alpha=.82)$ was strong. Internal consistency for the communication openness scale was improved $(\alpha=$ .71) by deleting one question.

Despite the removal of questions to improve scale consistency, reliabilities were 
still lower than anticipated. An editorial by Ajzen (2011) described the limits of the predictive utility of the TPB, stating that reliability coefficients in excess of 0.75 are rarely observed and values around 0.6 are more realistic. Lower correlation coefficients $(0.40-0.57)$ were reported in a meta-analysis by Rivis \& Sheeran (2003), suggesting the results of this study are consistent with published data.

\section{Psychological Safety}

Psychological safety summary scores were calculated to assess if participants felt safe taking interpersonal risks on their workplace team (or unit). Low scores were reflective of poor psychological safety and suggested participants did not feel safe engaging in interpersonally risky behavior. Data were explored for normality and outliers. Summary scores were converted to $Z$ score, and values greater than 3 or less than -3 were considered outliers. Based on this criteria, one data point was excluded from the analysis. Data were found to be negatively skewed, and the Kolmogorov-Smirnov test of normality indicated that psychological safety summary scores did not follow a normal distribution, $D(81)=.11, p=.02$. Given the sample size of this study, a violation of normality was not considered problematic (Ghasemi \& Zahediasl, 2012).

Psychological safety raw scores were stratified into three levels (high, moderate, low). Low scores were defined as $-1 \mathrm{SD}$, and high scores were +1SD. Overall, participants $(75$ percent) reported moderate levels of psychological safety $(M=26, S D=$ 5.5). Psychological summary scores were examined by practitioner group (physicians, practitioners, nurses). Practitioners (physician assistants and nurse practitioners) reported 
the highest psychological safety $(M=27.84, S D=4.17)$. Nurses $(M=25.28, S D=6.14)$ and physicians $(M=25.17, S D=5.65)$ had very similar scores. A one-way ANOVA found no significant differences between the groups, $F(2,79)=1.96, p=.15$.

Psychological safety summary scores were also compared between health care professionals working in high-risk $(M=26.16, S D=5.97)$ versus moderate/low risk $(M=$ 25.90, $S D=5.26)$ specialties using an independent samples t-test and no significant differences were found $(p=.84)$. Psychological safety was correlated with several variables, including the safety culture subscale scores of communication openness, $r(79)$ $=.41, p<.001$ and safety grade ratings, $r(82)=.53, p<.001$. Psychological safety was highly correlated with the intention to report $(r=.24, p=.029)$, meaning the intention to report increased as psychological safety increased and was also significantly correlated with attitude toward the behavior $(r=.31, p=.005)$ and subjective norms $(r=.33, p=$ .002). The correlation between perceived behavioral control and psychological safety was not significant.

\section{Theory of Planned Behavior}

The theory of planned behavior predictor variables included behavioral attitude, subjective norms, perceived behavioral control, and intention. A correlation matrix showed all variables were significantly correlated with intention, with several other correlations significant at the $p<.001$ level (see Table 2). 


\section{Table 2}

Correlations between TPB Constructs \& Intention to Report

\begin{tabular}{|c|c|c|c|c|}
\hline Measure & 1 & 2 & 3 & 4 \\
\hline Intention & 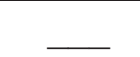 & & & \\
\hline Attitude & $.459 * *$ & 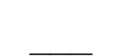 & & \\
\hline Subjective Norms & $.484 * *$ & $.491 * *$ & $\longrightarrow$ & \\
\hline $\begin{array}{l}\text { Perceived Behavioral } \\
\text { Control }\end{array}$ & $.519^{* *}$ & .199 & $.378 * *$ & 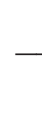 \\
\hline
\end{tabular}

** Correlation is significant at the 0.01 level (2-tailed)

Intention summary scores ranged from 3 to 15 , with a mean score of $M=13.24$. Higher scores reflected a stronger intention to report. Over half of the sample (54.4 percent) had an intention summary score of 15 , which was the maximum score possible. This likely represents a ceiling effect and suggests the presence of a social desirability bias. Intention scores did not significantly differ across positions $F(2,86)=1.36, p=.26$. Nurses and practitioners had nearly identical scores, $M=13.43(\mathrm{SD}=2.67)$ and $M=$ $13.63(\mathrm{SD}=2.65)$, while physician intention scores were lower $(M=12.52, S D=2.43)$. Subjective norm summary scores ranged from 3 to 10 , with a mean score of $M=$ 7.77, $\mathrm{SD}=1.8$. Higher scores reflected increased social pressure to report from important people. The one-way ANOVA, $F(2,86)=3.46, p=.036$ demonstrated statistically significant differences in subjective norm scores across positions. Tukey post hoc testing revealed that nurses reported greater social pressure to report errors $(M=$ 
$8.19, S D=1.83)$ compared to physicians $(M=7.0, S D=1.81)$. Subjective norm summary scores for the practitioner group were $M=7.89, S D=1.72$.

\section{Table 3}

TPB Predictors Summary Scores

\begin{tabular}{lll}
\hline Predictor Variables & $N$ & $M(S D)$ \\
\hline Intention & 90 & $13.24(2.6)$ \\
Behavioral Attitude & 88 & $13.83(1.76)$ \\
Subjective Norm & 90 & $7.77(1.83)$ \\
Perceived Behavioral Control & 90 & $8.34(1.57)$ \\
\end{tabular}

An analysis was conducted on the survey item assessing subjective norms of a descriptive nature. Descriptive norms measure what important people actually do versus what people think they should do (injunctive norms). Nearly 60 percent of the sample endorsed that people important to them (in the work environment) actually report errors, while over 20 percent strongly indicated their peers do not report. When examined across groups, noteworthy differences emerged between nurses and physicians. Only 31 percent of physicians agreed (somewhat or strongly) that their colleagues (or people in the workplace who are important to them) actually reported medical errors. This is compared to nurses and practitioners, which were both close to 60 percent actual engagement in reporting behavior by peers. Differences in the percent that endorsed (somewhat or strongly) that their peers would not report across groups were not statistically significant. The practitioner group indicated less than 10 percent of peers do not actually engage in 
reporting, compared to 21 percent of nurses, and 27 percent of physicians. Physicians were disinclined at a much higher rate than nurses and practitioners to infer or pass judgement on the behavior of their peers, as evidenced by close to 35 percent choosing not to respond.

Behavioral Attitude summary scores ranged from 3 to 15 , with a mean score of $M$ $=13.83, S D=1.76$. Higher scores reflected a more positive attitude towards reporting. Significant differences were found between groups using ANOVA, $F(2,84)=11.12, p<$ .001 . Physicians reported a significantly lower or more negative attitudes $(p<.001)$ towards reporting medical errors $(M=12.56, S D=2.47)$ compared to nurses $(M=14.29$, $S D=1.07)$ or practitioners $(M=14.41, S D=1.05)$.

Perceived behavioral control summary scores ranged from 3 to 10, with a mean score of $M=8.34$. Higher scores represented a greater level of control over the behavior of reporting errors. An ANOVA revealed no significant differences between groups, $F$ $(2,86)=.10, p=.907$. Mean scores were uniform across groups, physicians $(M=8.31$, $S D=1.62)$, nurses $(M=8.33, S D=1.62)$, and practitioners $(M=8.48, S D=1.48)$.

Two questions were asked to get at actual behavioral control. Participants were asked if they knew how to report a medical error and if they knew what kind of errors to report. A majority of participants (87 percent) indicated knowing what type of errors to report, and 89 percent reported knowing how to report. A bivariate correlation between the two variables was strong $(r=.78)$ and significant at the $\mathrm{p}<0.001$ value. Only seven percent of participants indicated not knowing how to report, nine percent did not know what type to report, and four percent marked unsure on both questions. 
Participants received a score for knowing both how and what to report. A frequency analysis showed nearly three fourths of the sample (74 percent) received a perfect score. In contrast, 9 percent of participants $(n=8)$ received a failing score $(\leq 50$ percent). A breakdown of the data revealed those receiving a failing score were either a nurse (67 percent) or nurse practitioner (33 percent). All physicians and physician assistants in this study received a perfect score. Registered nurses were the only respondents who reported mixed yes/no responses. Crosstab analysis of procedural reporting knowledge scores by position showed no significant association between groups.

$\underline{\text { Intention Simulation }}$

Intention simulation methodology required participants to make a behavioral (yes/no) decision. Participants were asked to read a case study and decide if an error had occurred, and if so, whether they would report it. The number of "yes" answers were tabulated and served as an intention simulation score. Higher scores reflected a stronger intention to report. All cases contained reportable errors. The mean intention simulation score for the sample was $\mathrm{M}=5.03, \mathrm{SD}=1.15$, out of a possible six points maximum. Over 64 percent of respondents completed all six case studies.

Results of a one-way ANOVA revealed statistically significantly differences between positions, $F(2,56)=4.33, p=.02$. Post hoc testing identified significant differences between physicians and nurses $(p=.02)$. Intention simulation scores for physicians were significantly lower $(M=4.39, S D=1.61)$ compared to nurses $(\mathrm{M}=5.33$, $\mathrm{SD}=.70)$. No statistically significant differences were found in intention simulation 
scores between the three levels of psychological safety, $F(2,57)=0.81, p=.45$.

Participants $(n=9)$ working in environments with low psychological safety had lower mean intention simulation scores $(M=4.89, S D=1.45)$ compared to those $(\mathrm{n}=44)$ in environments with moderate psychological safety $(\mathrm{M}=5.14, \mathrm{SD}=.979)$ and those $(\mathrm{n}=$ 7) in environments with high psychological safety $(\mathrm{M}=4.57, \mathrm{SD}=1.72)$.

Given that not all participants completed all six case studies, a posthoc power analysis was conducted using $G^{*}$ Power to assess whether this portion of the study was adequately powered to detect significance differences. Results indicated the study was powered at the 0.60 level, with a total sample size of 72 , three groups, and a 0.3 effect size. The lack of power for this section of the study should be noted as a significant limitation and results should be interpreted with care.

\section{Colleague Intention Simulation}

In absence of a formal measure of Social Desirability Bias (SDB), participants were asked to state whether they believed their colleagues would report the error outlined in each case study. SDB would be considered present if participants reported their behavioral intentions in a more positive manner than prevailing social norms. The number of "yes" answers were tabulated and served as the colleague intention simulation score. Mean scores for colleague intention simulation were $M=4.60, S D=1.48$. Correlation between the two intention simulation scores (self and colleague) was significant, $r(60)=.768, p<.001$. Colleague intention simulation scores were examined by position to assess for any hierarchical differences. A oneway ANOVA found significant differences in scores between positions, $F(2,56)=3.01, p=.05)$. Scores were 
the lowest for physicians $(M=3.94, S D .=1.80)$ compared to practitioners $(M=4.65, S D$ $=1.17)$ and nurse $(M=5.04, S D .=1.30)$. Across the board, all practitioners reported their behavioral intention to report in a more positive manner than their colleagues, suggesting the presence of SDB. Figure 7 shows the difference between measures of intention simulation to highlight the degree to which participants reported their behavior more favorably than their colleagues. These results highly suggest the presence of social desirability bias was present in this study.

\section{Figure 7}

Intention to Report Medical Errors: Perceptions of Self and Colleague Behavior

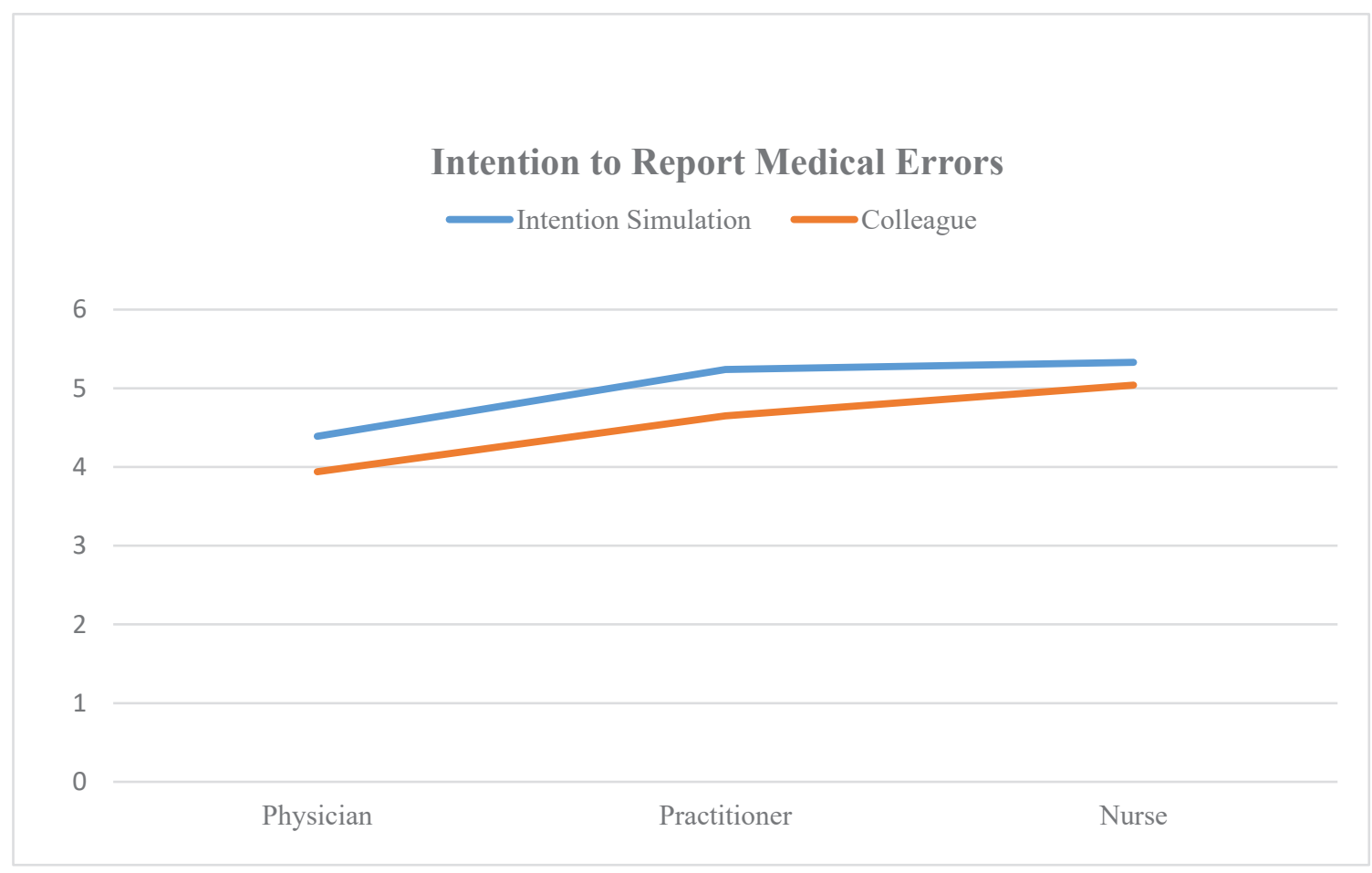


No significant differences were found in intention scores across the three levels of psychological safety. Participants working in environments with low psychological safety had lower mean colleague intention simulation scores $(M=4.11, S D=2.21)$ compared to those in environments with moderate psychological safety $(M=4.73, S D=1.26)$ and high environments $(M=4.43, S D=1.72)$. Table 8 provides a summary of responses (percent) to each question (error recognition, intention simulation, colleague intention simulation) across all six case studies.

\section{Table 4}

Participant Responses to Case Studies

\begin{tabular}{|c|c|c|c|c|c|c|}
\hline & \multicolumn{2}{|c|}{$\begin{array}{l}\text { Error Occur? } \\
(\%)\end{array}$} & \multicolumn{2}{|c|}{$\begin{array}{l}\text { Self-Report? } \\
\text { (\%) }\end{array}$} & \multicolumn{2}{|c|}{$\begin{array}{l}\text { Colleague Report? } \\
\text { (\%) }\end{array}$} \\
\hline & $\underline{\text { Yes }}$ & No/Maybe & $\underline{\text { Yes }}$ & $\underline{\text { No/Unsure }}$ & $\underline{\text { Yes }}$ & $\underline{\text { No/Unsure }}$ \\
\hline Case \#1 & 92 & 8 & 81 & 19 & 73 & 27 \\
\hline Case \#2 & 72 & 28 & 74 & 26 & 60 & 40 \\
\hline Case \#3 & 100 & - & 97 & 3 & 95 & 5 \\
\hline Case \#4 & 54 & 46 & 70 & 30 & 58 & 42 \\
\hline Case \#5 & 77 & 23 & 83 & 17 & 77 & 23 \\
\hline
\end{tabular}


$\begin{array}{lllllll}\text { Case \#6 } & 99 & 1 & 99 & 1 & 96 & 4\end{array}$

\section{Correct Decision Scores}

A correct decision score was calculated from participant responses to the case study scenarios. A correct decision was awarded to individuals who answered yes to both questions asking if an error occurred and if they would report it. Individuals who answered yes - an error had occurred, but indicated they would not report the error were coded as making an incorrect decision. Participants who answered negatively, that they did not believe an error had occurred, were marked as an incorrect decision. Average correct decision score was $M=4.4, S D=1.32$. Slightly over one quarter of the sample made the correct decision in all six cases. In contrast, over half the sample received failing scores of 67 percent or below. Scores were consistent across the three groups; physicians $(M=4.04, S D=1.49)$, practitioners $(M=4.41, S D=1.14)$, and nurses $(M=$ $4.59, S D=1.29$ ). Decision scores by position can be seen in Table 9 .

\section{Table 5}

Correct Decision Scores (1-100\%)

\begin{tabular}{llll}
\hline & Physician (\%) & Nurse (\%) & Practitioner (\%) \\
\hline $100 \%(6 / 6)$ & 26 & 28 & 18 \\
$83 \%(5 / 6)$ & 9 & 31 & 32 \\
$<67 \%$ - Failing & 65 & 41 & 50
\end{tabular}




\section{RESEARCH QUESTIONS}

Multiple linear regression analyses were used to answer the following research questions and corresponding hypotheses. All assumptions of linear regression were evaluated and fulfilled as described in the methods sections prior to data analysis. Results are as follows:

\section{Question 1}

Research Question 1(a) - What is the relationship between attitude towards behavior, subjective norms, perceived behavioral control and the intention to report medical errors?

Hypothesis 1: The theory of planned behavior will significantly predict the intention to report medical errors.

Results: Regression model statistically significantly explained $41.4 \%$ of the variance in intention scores, $R^{2}=.414, F(3,82)=19.31, p<.001$.

Decision: Null hypothesis was not rejected.

The first research question (1a) evaluated the relationship between the theory of planned behavior's antecedents of behavior (attitude towards behavior, subjective norms, and perceived behavioral control) and the intention to report medical errors using multiple linear regression. A scatterplot of predicted standardized regression values (xaxis) and standardized regression residual values (y-axis) did not show violations of 
homoscedasticity, independence, or linearity. Variance inflation factor (VIF) values were within acceptable ranges (1.04-1.48) for all independent variables, suggesting no issues with multicollinearity.

Model statistics indicated the three antecedents of behavior explained 41.4 percent of the variance in intention scores, $R^{2}=.414, F(3,82)=19.31, p<001$. Attitude towards behavior $(\beta=.44, p=.003)$ and perceived behavioral control $(\beta=.64, p<.001)$ were significant predictor variables. The construct of subjective norms, made up of injunctive and descriptive norms, was not a significant predictor. Although zero-order correlations were highly significant $(r=.484, p<.001)$, part and partial correlations revealed the correlation was substantially reduced when controlling for attitude and perceived behavioral control, $r=.134$.

Research Question 1(b) - Does the inclusion of additional predictor variables explain additional variance in intention scores?

Hypothesis 2: Inclusion of background variables, past reporting behavior, and actual behavioral control to the TPB model will significantly predict intention scores.

Results: Regression model was statistically significant in explaining $53.3 \%$ of the variance in intention scores, $R^{2}=.53, F(8,69)=9.86, \mathrm{p}<.001$.

Decision: Null hypothesis was not rejected

A second model was constructed to determine if including additional background 
predictor variables would increase the explanatory power of the model. In the first step actual behavioral control (knowing how and what to report) was added and found to be a significant predictor variable, adding an additional six percent in explained variance, $R^{2}=$ $.474, F(4,77)=17.31, p<.001$. To further improve model statistics, additional background factors of age, position, risk, and reporting behavior were added. This final model contained eight predictor variables and explained 53.3 percent of the variance in intention to report medical errors, $R^{2}=.533, F(8,69)=9.86, p<.001$ (see Table 4).

\section{Table 6}

Results of the Multiple Linear Regression Analyses

\begin{tabular}{lccccccc}
\hline Variable & $\mathrm{t}$ & $\mathrm{p}$ & $\beta$ & $\mathrm{F}$ & $\mathrm{df}$ & $\mathrm{p}$ & $\mathrm{R}^{2}$ \\
\hline Constant & & & & 9.86 & 8,69 & $<.001$ & .533 \\
Attitude & 3.01 & .004 & .458 & & & & \\
Subjective Norms & 1.30 & .199 & .198 & & & & \\
Perceived Behavioral Control & 3.26 & .002 & .560 & & & & \\
Actual Behavioral Control & 2.84 & .006 & 1.19 & & & \\
Risk Grouping & .045 & .965 & .021 & & & & \\
Age & .805 & .423 & .017 & & & & \\
Position/Hierarchy & .474 & .637 & .146 & & & \\
Past Reporting Behavior & 2.29 & .025 & .028 & & & \\
\hline *Intention (Generalized) as the criterion variable & & & & & & \\
\hline
\end{tabular}




\section{Question 2}

Research Question 2(a) - What is the Relationship between Psychological Safety and Reporting Behavior?

Hypothesis 1: Psychological safety will be a significant predictor of the intention to report medical errors.

Results: Psychological safety explained $6 \%$ of the variance in intention scores, $R^{2}$ $=.06, F(1,78)=4.95, p=.029$.

Decision: Null hypothesis was not rejected

The second research question evaluated the relationship between psychological safety and the intention to report medical errors. A correlation matrix was constructed to examine the relationship between psychological safety and the constructs of the theory of planned behavior. Results demonstrated that psychological safety was significantly correlated with intention $(r=.244, p<.05)$, subjective norms $(r=.343, p<.001)$, and attitude $(r=.31, p<.001)$ (see Table 5). A simple linear regression examined the proportion of variance in intention that could be explained from psychological safety scores. Results showed that psychological safety predicted six percent of the variance in intention, $R^{2}=.06, F(1,78)=4.95, p=.029$. 


\section{Table 7}

Correlations between Psychological Safety \& TPB Constructs

\begin{tabular}{|c|c|c|c|c|c|}
\hline Measure & 1 & 2 & 3 & 4 & 5 \\
\hline Psychological Safety & 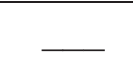 & & & & \\
\hline Intention & $.244^{*}$ & 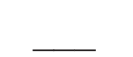 & & & \\
\hline Subjective Norms & $.343 * *$ & $.484 * *$ & 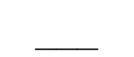 & & \\
\hline Attitude & $.312 * *$ & $.459 * *$ & $.491 * *$ & $\underline{-}$ & \\
\hline $\begin{array}{l}\text { Perceived Behavioral } \\
\text { Control }\end{array}$ & .186 & $.519 * *$ & $.378 * *$ & .199 & \\
\hline
\end{tabular}

Research Question 2(b) - What is the relationship between TPB antecedents of behavior, psychological safety, and the intention to report medical errors?

Hypothesis 2: The inclusion of psychological safety as an additional predictor variable in the theory of planned behavior will significantly improve explanatory power of the model.

Results: Regression model was statistically significant in predicting intention scores, explaining $36.9 \%$ of the variance in intention scores. However, psychological safety was not a significant predictor.

Decision: Reject the null hypothesis 
Research question ( $2 b$ ) evaluated the relationship between the antecedents of behavior, psychological safety, and the intention to report. Linear regression model statistics were statistically significant in predicting intention scores, explaining $36.9 \%$ of the variance in intention scores, $R^{2}=.369, F(4,74)=10.82, \mathrm{p}=<.001$. However, psychological safety was not a significant predictor as evidenced by low and nonsignificant coefficients $(\beta=.01, p=.78)$.

Research Question 2(c) - Does the inclusion of psychological safety in an expanded model explain additional variance in intention scores?

Hypothesis 3: Inclusion psychological safety in an expanded model of the TPB would significantly predict intention scores.

Results: Regression model statistically significant. Explained $47.9 \%$ of the variance in intention scores, $R^{2}=.414, F(9,61)=6.22, \mathrm{p}<.001$. However, psychological safety was not a significant predictor.

Decision: Reject the null hypothesis

A second regression model integrating the constructs of the theory of planned behavior explained 43.4 percent of variance in intention scores, $R^{2}=.434, F(5,69)=$ $10.59, p=<.001$. Constructs of behavioral attitude $(\beta=.54, p=.001)$, perceived behavioral control $(\beta=.59, p=.002)$, and actual behavioral control $(\beta=1.25, p=.005)$, contributed significantly to the model statistics (see Figure 7). 
Figure 8

Regression Path Analysis

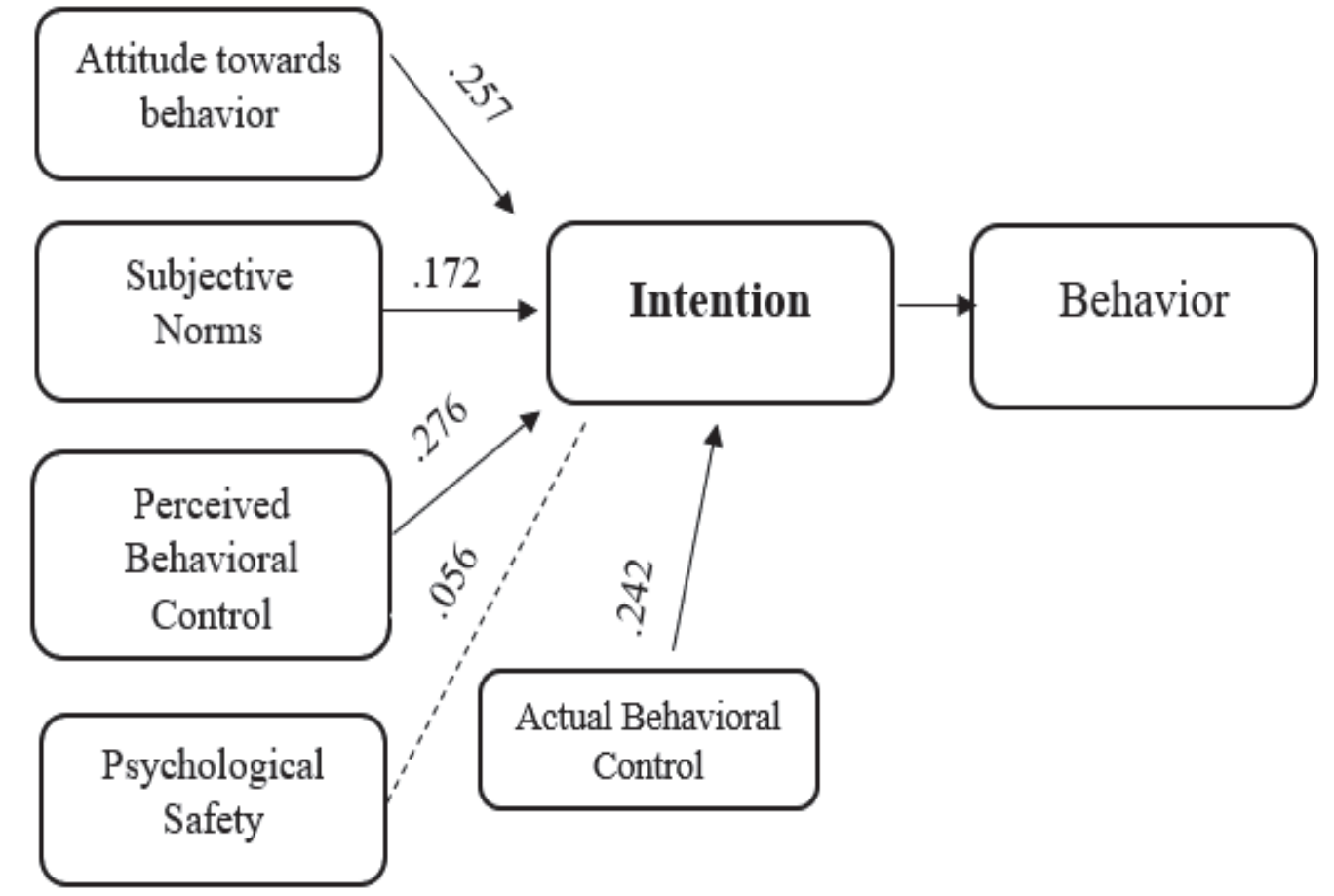

\section{Question 3}

Research Question 3 - How does psychological safety influence the intention to report?

Hypothesis 1: Psychological safety will independently influence the intention to report errors.

Results: A Baron \& Kenny analysis did not find evidence of mediation.

Decision: Reject the null hypothesis 
Research Question 3 used the Baron and Kenny (1986) approach to evaluate if psychological safety served as a mediator or moderator in the relationship between the theory of planned behavior constructs and the intention to report. Subjective norms were the first construct examined in the mediation analysis. The initial step regressed subjective norm scores on intention, without the mediator (psychological safety); this regression was significant, $b=.689, t(87)=5.16, p=<.001$.

The second step showed that the regression of subjective norms on the mediator, psychological safety, was significant, $b=1.05, t(78)=3.23, \mathrm{p}=.002$. The third step showed that the regression of the mediator, controlling for subjective norm, on intention was not significant, $b=.050, t(76)=.994, \mathrm{p}=.324$. In the fourth step, psychological safety was assessed as a mediator in the relationship between subjective norms and intention. Subjective norm scores remained a significant predictor of intention scores, $b=$ $.568, t(76)=3.65, \mathrm{p}=<.001$, when the effects of psychological safety were controlled. Evidence of mediation would have shown a significant reduction. A Sobel test did not confirm the existence of mediation of psychological safety on the relationship between subjective norms and the intention $(z=.935, p=.350)$.

Perceived behavioral control was the second construct examined in the mediation analysis. The initial step regressed perceived behavioral control on intention, without the mediator (psychological safety); this regression was significant, $b=.856, t(87)=5.67, p$ $=<.001$. The second step showed that the regression of perceived behavioral control on the mediator, psychological safety, was not significant, $b=.693, t(78)=1.67, p=.099$. The third step showed that the regression of the mediator, controlling for perceived 
behavioral control, was also not significant, $b=.077, t(76)=1.61, p=.113$. The fourth step assessed whether psychological safety completely mediated the relationship between perceived behavioral control and intention. Perceived behavioral control remained a significant predictor of intention scores, $b=.718, t(76)=4.06, p=<.001$, suggesting no mediation. A Sobel test was conducted and did not find mediation $(z=1.15, p=.251)$.

Attitude was the third and final construct of the theory of planned behavior tested for mediation. The initial step regressed attitude on intention, without the mediator (psychological safety); this regression was significant, $b=.672, t(85)=4.76, p<.001$. The second step showed that the regression of attitude on the mediator was significant, $b$ $=.99, t(79)=2.92, p=.005$. The third step showed that the regression of the mediator, controlling for attitude, was not significant, $b=.05, t(77)=1.00, p=.320$. Step Four of the analyses revealed that, controlling for the mediator (psychological safety), attitude scores were still a significant predictor of intention scores, $b=.64, t(76)=4.10, p=$ $<.001$. A Sobel test confirmed there was no mediation $(z=.95, p=.344)$.

To test the hypothesis that the intention to report medical errors is a function of multiple risk factors, and more specifically whether psychological safety moderates the relationship between subjective norms and intention, a hierarchical multiple regression analysis was conducted. The first model included the variables of subjective norms and psychological safety. These variables accounted for a significant amount of variance in intention, $R^{2}=.198, F(2,76)=9.41, p<.001$. Next, the interaction term between subjective norms and psychological safety was added to the regression model, which did not account for a significant proportion of the variance in intention, $\Delta \mathrm{R}^{2}=.036, \Delta \mathrm{F}(1$, 
$75)=3.55, p=.57$. The effect of subjective norms on intention was positive and significant $(b=.50$, s.e. $=.16, p=.002)$, however, the effect of psychological safety was not significant $(b=.03$, s.e. $=.05, p=.502)$. This suggests that psychological safety was not a significant moderator of the effects of subjective norms on intention.

A second hierarchical multiple regression analysis was conducted to test whether psychological safety moderated the relationship between perceived behavioral control and intention. Perceived behavioral control and psychological safety accounted for a significant amount of variance in intention scores, $\mathrm{R}^{2}=.226, F(2,76)=11.11, p<.001$. The interaction term between perceived behavioral control and psychological safety was added to the regression model, which did not account for a significant proportion of the variance in intention, $\Delta \mathrm{R}^{2}=.024, \Delta \mathrm{F}(1,75)=2.37, \mathrm{p}=.128,(\mathrm{~b}=.04$, s.e. $=.03, \mathrm{t}(75)=$ $1.54, \mathrm{p}=.128$. The effect of perceived behavioral control on intention was positive and significant $(b=.77$, s.e. $=.18, \mathrm{p}<.001$. The effect of psychological safety was positive, but not significant $(\mathrm{b}=.09$, s.e. $=.05, \mathrm{p}=.05)$. This suggests that psychological safety was not a significant moderator of the effects of perceived behavioral control on intention.

A third and final hierarchical multiple regression analysis was conducted to examine the relationship between attitude and intention. Attitude and psychological safety accounted for a significant amount of variance in intention scores, $\mathrm{R}^{2}=.228, \mathrm{~F}(2$, $77)=11.39, \mathrm{p}<.001$. The interaction term did not account for a significant proportion of the variance in intention, $\Delta R^{2}=.003, \Delta F(1,76)=.329, p=.57,(b=-.01$, s.e. $=.02, t(76)$ $=-.57, p=.568$. The effect of attitude on intention was positive and significant $(b=.59$, 
s.e. $=.18, p=.002$. The effect of psychological safety was positive, but not significant (b $=.05$, s.e. $=.05, p=.342$ ). This suggests that psychological safety was not a significant moderator of the effects of attitude on intention.

\section{Question 4}

Research Question 4 - Can an alternative measure of intention better approximate actual behavior?

Hypothesis 1: Intention simulation will serve as a better proxy for actual behavior than generalized intention in predicting error reporting behavior.

Results: Intention simulation did not perform better than generalized intention in any regression models.

Decision: Reject the null hypothesis

The final research question looked to assess if a measure of intention simulation would serve as a better proxy for actual behavior compared to the more commonly used measure of generalized intention. Bivariate correlations between intention simulation and generalized intention were not significant, $r(60)=.10, p=.45$. A correlation matrix was constructed to examine the associations between intention simulation, the constructs of the theory of planned behavior, and psychological safety. Significant associations shown in table 7 were found between intention simulation and attitude $(p<.001)$ and subjective norms $(p=.012)$. 


\section{Table 8}

Correlations between TPB Constructs \& Intention Simulation

\begin{tabular}{|lccccc|}
\hline Measure & 1 & 2 & 3 & 4 & 5 \\
Intention Simulation & - & & & \\
Subjective Norms & $.323^{*}$ & - & & & \\
Attitude & $.533^{* *}$ & $.491^{* *}$ & -199 & -186 & - \\
$\begin{array}{l}\text { Perceived Behavioral } \\
\text { Control }\end{array}$ & .147 & $.378^{* *}$ & $.192 * *$ & \\
Psychological Safety & .090 & $.343^{* *}$ & $.312 *$ & \\
\hline
\end{tabular}

** Correlation is significant at the 0.01 level (2-tailed)

Linear regression was used to test how well the constructs of the theory of planned behavior predicted intention simulation. Model statistics indicated the three predictors variables explained 28.9 percent of the variance in intention simulation scores $\left(R^{2}=.289, F(3,56)=7.58, p<.001\right)$. Attitude towards behavior $(\beta=.50, p=<.001)$ was the only significant predictor. Additional background factors of age, position, risk grouping, and reporting behavior were added to the final model that contained eight predictor variables and explained 31.5 percent of the variance in intention to report medical errors, $R^{2}=.315, F(8,44)=2.52, p=.024$. 
CHAPTER V

DISCUSSION

Reporting can be conceptualized as a complex health behavior that requires individuals to both self-incriminate and hold accountable their colleagues and superiors in the name of patient safety. Using a well-established model of human behavior, this theory-driven study aimed to better understand medical error reporting behavior in a random sample of health care professionals licensed in the state of Kentucky. This chapter expands on the findings from Chapter IV and is followed by a discussion of the limitations and broader public health implications.

\section{Discussion of Research Findings}

The results indicated that conceptualizing reporting (or the intention to report) through the constructs of the Theory of Planned Behavior (TPB) explained a significant proportion of the variance in an individual's intention to report medical errors and revealed the relative importance and significance of each predictor variable. Results did not support the addition of psychological safety to the model as it did not significantly improve the explanatory power of the model. Lastly, the use of an alternate measure of behavioral intention, intention simulation, derived from case study scenarios did not 
serve as a better proxy for actual behavior as evidenced by reduced model statistics. The following is a discussion of these results and their implications for public health.

The participants in this study represented a self-selected sample of health care providers licensed to practice in the state of Kentucky and included physicians, nurses, nurse practitioners, and physician assistants. Nurses made up the majority (40 percent) of the sample, followed by physicians (30 percent), while nurse practitioners and physician assistants were grouped into a third category for comparison. An important outcome of this study was to better understand the influence of hierarchy on reporting behavior, both between and within groups. However, for reasons that are unclear, no licensed practical nurses (LPNs) participated in the study, despite being included in the sampling pool. LPNs are lower status employees and are likely to spend the majority of work time on the floor; perhaps their lack of participation was due to having less access to a computer during the workday. This study also did not offer any incentive for survey completion, which may have further precluded participation. Similarly, only one medical resident/fellow attempted the study, making any within group differences difficult to explore.

The study met the minimum required sample size of $(\mathrm{N}=85)$ to adequately power the study. A priori power analyses recommended a sample size of 85 and roughly 91 participants were included in the primary analyses. Survey response rates were lower than expected and suggest that alternative recruitment strategies may be more cost effective. An email marketing company approach to study recruitment was intended to mitigate threats of risk related to disclosing sensitive or personal information by making 
the survey completely anonymous and random. This approach was also intended to mitigate social desirability bias, Future studies should explore a mix of electronic, paper, and phone surveys with adequate follow up to improve response rates in the busy population.

Questions about past reporting behavior revealed that 56 percent of participants made no reports during the past 12 months. These results were similar to data published in the Agency for Health Care Research and Quality's (AHRQ) comparative database which report 55 percent of all participants made no reports. A rate of underreporting, obtained by asking participants how many errors they committed or observed over the same 12 months, showed nearly 20 percent did not report an error when in fact they should have. Physicians had the highest rate of underreporting at 26 percent, followed by nurses. These numbers are dramatically higher than published data that reports pervasive rates of underreporting in excess of 90 percent. These differences suggest the presence of a strong social desirability bias, and therefore, results should be interpreted with care.

\section{$\underline{\text { Research Questions }}$}

The first research question explored in the relationship between the antecedents of behavior and the intention to report medical errors. Linear regression techniques were used to build a model that showed the proportion of variance in intention explained by the predictor variables of attitude, subjective norms, and perceived behavioral control. The model accounted for 41.4 percent of variance in intention scores, slightly above the 19 to 40 percent range reported by a meta-review of over 200 data sets (Rivis \& Sheeran, 
2003). Perceived behavioral control and attitude were stronger predictors than normative beliefs. The addition of other variables, including background factors, increased the explanatory power of the final regression model to 53.3 percent.

The second research question explored the relationship between psychological safety and intention. Considered alone, psychological safety predicted six percent of the variance in intention. However, when psychological safety was added to the larger TPB regression model, the coefficient of determination decreased from 53.3 percent to 47.9 percent. Model coefficients confirmed psychological safety was not a significant predictor $(p=.994)$ in the full regression model.

These findings suggested psychological safety may be redundant with the TPB's existing predictors, working through constructs such as behavioral attitude, which was significantly correlated with psychological safety, $r(81)=.312, p=.005$, or subjective norms, $r(80)=.343, p=.002$, as predicted by Ajzen (2006). These results were unexpected given the strong links between psychological safety, culture and reporting behavior; a strong predictive relationship was anticipated. Although the effects of psychological safety were not actualized in this study, responses to open-ended comments suggest it is alive and present. A 58-year-old, obstetric physician said, "Your questions are too limiting and don't reflect what is going on in the real world of medicine". A 64-year-old psychiatric nurse practitioner said "There is a lot of retaliation towards those who speak up or document their concerns". Another nurse wrote, "I was fired in retaliation for my numerous reports of substandard patient care, with my employer". 


\section{$\underline{\text { Limitations }}$}

There are limitations to this study that must be acknowledged and taken into consideration when interpreting the results. The first limitation is a potential sampling bias. An outside health care marketing company was hired to help recruit a random sample of healthcare professionals. Hiring a marketing company allowed for a targeted email campaign and included access to the AMA Physician Masterfile that would have otherwise been unavailable. Random sampling occurred within this population framework, which may not be a true representation of the population of interest. Individuals who opt-in to receive email solicitations may have meaningful differences (in respect to reporting errors) compared to those who opt-out of receiving such notifications. These differences may influence the way in which participants responded to the questions, introducing response bias. This study did not provide compensation for participation, which may have precluded individuals who were interested in taking the survey, but not willing or able to commit their time and effort. Moreover, the health care professionals who responded to this study may just be more intrinsically motivated towards patient safety compared to those who chose not to respond.

\section{$\underline{\text { Social Desirability Bias }}$}

The presence of a social desirability bias is suspected in this study and its influence on the data merits discussion. This study asked questions that have the potential to be self-incriminating and/or embarrassing. Answering these questions truthfully could, therefore, be considered risky to individuals who fear retaliation or repercussions. Although efforts were taken to ensure confidentiality and anonymity, it is possible that 
participants answered questions in a manner which portrayed themselves favorably. This bias is most suspected in the rate of underreporting observed in this study, which was considerably lower (17 percent) than previous studies ( $80-90$ percent) (Barach \& Small, 2000). This difference was not unexpected; the methodology used in published studies, including retrospective record reviews and software mining tools, was superior at finding actual reporting behavior, while this study relied on self-reports in hypothetical situations. The extent to which these values differed, however, was unexpected. An article by Fisher (1993) stated that the extent to which social desirability bias influences a response reflects the relevant importance of that value within society. This would suggest participants in this study placed high importance on their role in patient safety.

Additional limitations included the use of self-report measures and a lengthy survey. Self-report measures have well-known limitations, but are critical to behaviors that are otherwise unobservable (Garcia \& Gustavson, 2000). Although the survey took on average 15 minutes to complete, several participants left open-ended comments stating they felt the survey was too long. The response burden associated with completing this study was projected to be in the responses to the case studies in the second section of the survey, which required participants to process technical information and make clinical judgements. Response fatigue was observed in this study through both direct comments and a steady decline in responses to the case study scenarios.

There are also published limitations and warnings about attempting a measure of intention simulation, instructing researchers to "[prepare] with great care, or it may be misleading" (Jones, Gerrity, \& Earp, 1990). Collecting a measure of intention simulation 
was a secondary aim of this research study, therefore, previously published case study vignettes were used. Although these case studies are well-validated, they do not conform to the exact specifications outline by Francis (2004). Given these conditions the results of intention simulation analyses should be interpreted with caution. Post hoc power analysis confirmed the case study section of the study was not adequately powered to detect significant differences with power $=.0 .60$.

\section{Conclusions and Future Directions}

Medical errors are a public health epidemic and a major threat to patient safety and for reasons unclear, they do not get much attention in public health. The overarching goal of this study was to better understand the predictors of error reporting as to inform interventions for improved reporting and ultimately reduce medical errors. Reporting allows for errors, and the conditions in which they occur, to be identified and corrected. Reporting turns a threat into an opportunity for a corrective action.

This study used the TPB to investigate the influence of behavioral attitude, subjective norms, perceived behavioral control, and psychological safety on the intention to report medical errors. With the inclusion of background factors, over 53 percent of the variance in reporting behavior was explained by the model. Correlation and regression analysis found that perceived behavioral control was the most influential on the intention to report, followed closely by attitude towards behavior. Although results of statistical analyses did not find psychological safety to be a significant predictor, its role in error reporting behavior does warrant additional consideration. Results suggest significant ceiling effects resulting from social desirability bias, which appeared to be present in 
other areas as well, such as error reporting behavior.

Additional efforts to improve model statistics through the use of a novel measure of intention simulation did not result in any improvements. Future studies looking to use intention simulation as a proxy for behavior should follow the conceptual analysis review recommendations and warnings by Jones, Gerrity, and Earp (1990) very closely to be sure the results are valid. This study used existing validated case studies in place of developing unique scenarios, a strategy that did not result in improve model statistics.

This study also examined the influence of hierarchy on the intention to report. The health care industry has a deep-seated power structure and workers face a steep authority gradient when reporting. The results of this study suggest hierarchical differences were present but not fully revealed. Future studies should explore within group differences, looking at the hierarchical structure and norms within each profession, as well as institutional barriers.

Efforts to flatten the hierarchy, similar to steps taken by the aviation industry, could be transformative. However, none of these changes will be actualized unless all systems and clinicians value reporting enough to increase its occurrence. This study supports previous studies that suggest physicians are slightly disengaged from this behavior. Understanding the reasons and finding ways to involve physicians will be essential in safeguarding the health of the public through the reduction of medical errors. The airline industry did not transform their safety record without the full leadership and engagement of pilots.

Twenty plus years has passed since the Institute of Medicine published the To Err 
is Human report and the problems persist. Rigorous efforts to build a safer health system must identify and address the conditions that discourage healthcare professionals from reporting medical errors and turn these threats into opportunities to save hundreds of thousands of lives.

- You cannot fix that which is unknown 


\section{REFERENCES}

Abdulaziz, K., Brehaut, J., Taljaard, M., Emond, M., Sirois, M. J., Lee, J. S., . . Perry, J. J. (2015). National survey of physicians to determine the effect of unconditional incentives on response rates of physician postal surveys. BMJ Open, 5(2), e007166. doi:10.1136/bmjopen-2014-007166

Adler, L., Yi, D., Li, M., McBroom, B., Hauck, L., Sammer, C., . . Classen, D. (2018). Impact of Inpatient Harms on Hospital Finances and Patient Clinical Outcomes. Journal of Patient Safety, 14(2), 67-73. doi:10.1097/PTS.0000000000000171

AHRQ, A. f. H. R. a. Q. (July 2013, July 2017). Hospital Survey on Patient Safety Culture. Retrieved from http://www.ahrq.gov/professionals/quality-patientsafety/patientsafetyculture/hospital/index.html

Ajzen, I. (1991). The Theory of Planned Behavior. Organizational Behavior and Human Decision Processes, 50, 179-211. doi:10.1016/0749-5978(91)90020-T

Ajzen, I. (1991). The Theory of Planned Behavior. Organizational Behavior and Human Decision Processes, 50(2), 179-211. doi:Doi 10.1016/0749-5978(91)90020-T

Ajzen, I. (2011). The theory of planned behaviour: reactions and reflections. Psychol Health, 26(9), 1113-1127. doi:10.1080/08870446.2011.613995

Ajzen, I., \& Fishbein, M. (1980). Understanding attitudes and predicting social behaviour: Prentice-Hall.

Alsafi, E., Bahroon, S. A., Tamim, H., Al-Jahdali, H. H., Alzahrani, S., \& Al Sayyari, A. (2011). Physicians' Attitudes Toward Reporting Medical Errors-An Observational Study at a General Hospital in Saudi Arabia. Journal of Patient Safety, 7(3), 144147. doi:10.1097/PTS.0b013e31822c5a82 
Andel, C., Davidow, S. L., Hollander, M., \& Moreno, D. A. (2012). The economics of health care quality and medical errors. J Health Care Finance, 39(1), 39-50.

Retrieved from https://www.ncbi.nlm.nih.gov/pubmed/23155743

Anderson, J. G., \& Abrahamson, K. (2017). Your Health Care May Kill You: Medical Errors. Stud Health Technol Inform, 234, 13-17.

Angelis, A., Pancani, L., Steca, P., Colaceci, S., Giusti, A., Tibaldi, L., . . Vellone, E. (2017). Testing an explanatory model of nurses' intention to report adverse drug reactions in hospital settings. J Nurs Manag, 25(4), 307-317.

doi: $10.1111 /$ jonm. 12467

Antonow, J. A., Smith, A. B., \& Silver, M. P. (2000). Medication error reporting: a survey of nursing staff. J Nurs Care Qual, 15(1), 42-48. Retrieved from https://www.ncbi.nlm.nih.gov/pubmed/11008438

Appelbaum, N. P., Dow, A., Mazmanian, P. E., Jundt, D. K., \& Appelbaum, E. N. (2016). The effects of power, leadership and psychological safety on resident event reporting. Medical Education, 50(3), 343-350. doi:10.1111/medu.12947

Appiah, A. B., Tenkorang, E. Y., \& Maticka-Tyndale, E. (2017). Modeling Beliefs, Attitudes, and Intentions of Condom Use Among Secondary School Students in Kenya. Arch Sex Behav, 46(7), 1949-1960. doi:10.1007/s10508-017-0966-9

Ayodele, O. (2017). The Theory of Planned Behavior as a Predictor of HIV Testing Intention. American Journal of Health Behavior, 41(2), 147-151. doi:10.5993/AJHB.41.2.5

Barach, P., \& Small, S. D. (2000). Reporting and preventing medical mishaps: lessons from non-medical near miss reporting systems. BMJ, 320(7237), 759-763. Retrieved from https://www.ncbi.nlm.nih.gov/pubmed/10720361

Barter, C., \& Renold, E. (1999). The use of vignettes in qualitative research. Social research update, 25(9), 1-6.

Bates, D. W., Spell, N., Cullen, D. J., Burdick, E., Laird, N., Petersen, L. A., . . Leape, 
L. L. (1997). The costs of adverse drug events in hospitalized patients. Adverse Drug Events Prevention Study Group. JAMA, 277(4), 307-311.

Beasley, J. W., Escoto, K. H., \& Karsh, B. T. (2004). Design elements for a primary care medical error reporting system. Wmj, 103(1), 56-59.

Benoit-Smullyan, E. (1944). Status, status types, and status interrelations. American Sociological Review, 9, 151-161.

Brennan, T. A., Leape, L. L., Laird, N. M., Hebert, L., Localio, A. R., Lawthers, A. G., . . . Hiatt, H. H. (1991). Incidence of adverse events and negligence in hospitalized patients. Results of the Harvard Medical Practice Study I. N Engl J Med, 324(6), 370-376. doi:10.1056/NEJM199102073240604

Carroll, A. E., \& Buddenbaum, J. L. (2013). High and low-risk specialties experience with the U.S. medical malpractice system. BMC Health Services Research, 13(1), 465. doi:10.1186/1472-6963-13-465

Castel, E. S., Ginsburg, L. R., Zaheer, S., \& Tamim, H. (2015). Understanding nurses' and physicians' fear of repercussions for reporting errors: clinician characteristics, organization demographics, or leadership factors? BMC Health Serv Res, 15, 326. doi:10.1186/s12913-015-0987-9

Chassin, M. R. (2013). Improving the quality of health care: what's taking so long? Health Aff (Millwood), 32(10), 1761-1765. doi:10.1377/hlthaff.2013.0809

Chassin, M. R., \& Loeb, J. M. (2013). High-reliability health care: getting there from here. Milbank Q, 91(3), 459-490. doi:10.1111/1468-0009.12023

Chiang, H. Y., \& Pepper, G. A. (2006). Barriers to nurses' reporting of medication administration errors in Taiwan. J Nurs Scholarsh, 38(4), 392-399.

Cialdini, R. B., Reno, R. R., \& Kallgren, C. A. (1990). A focus theory of normative conduct: Recycling the concept of norms to reduce littering in public places. Journal of Personality and Social Psychology, 58(6), 1015-1026. doi:10.1037/0022-3514.58.6.1015 
Cohen, J. (1988). Statistical power analysis for the behavioral sciences (2nd ed. ed.). Hillsdale, N.J.: L. Erlbaum Associates.

Cook, \& Hoas. (2020). The Health Care Ethics Studies. Case Studies. Retrieved from http://www.umt.edu/bioethics/healthcare/resources/educational/casestudies/pscase studies/default.aspx

Cook, Hoas, H., Guttmannova, K., \& Joyner, J. C. (2004). An error by any other name. Am J Nurs, 104(6), 32-43; quiz 44.

Cook, R. I., Woods, D. D., \& Miller, C. (1998). National Patient Safety Foundation: A Tale of Two Stories: Contrasting Views of Patient Safety. Retrieved from Chicago, Il:

Creswell, J. W., Plano Clark, V. L., Gutmann, M., \& Hanson, W. (2003). Advanced Mixed Methods Research Designs. In C. T. A. Tashakkori (Ed.), Handbook of Mixed Methods in Social and Behavioural Research (pp. 209-240). Thousand Oaks, CA: Sage.

Derickson, R., Fishman, J., Osatuke, K., Teclaw, R., \& Ramsel, D. (2015). Psychological Safety and Error Reporting Within Veterans Health Administration Hospitals. Journal of Patient Safety, 11(1), 60-66. Retrieved from $<$ Go to ISI > //WOS:000349979700009

Dingley, C., Daugherty, K., Derieg, M. K., \& Persing, R. (2008). Advances in Patient Safety

Improving Patient Safety Through Provider Communication Strategy Enhancements. In K. Henriksen, J. B. Battles, M. A. Keyes, \& M. L. Grady (Eds.), Advances in Patient Safety: New Directions and Alternative Approaches (Vol. 3: Performance and Tools). Rockville (MD): Agency for Healthcare Research and Quality (US).

DMD Marketing Corporation. (2018). Products and Services. Retrieved from https://www.dmdconnects.com/products-services

Driskell, J., \& Salas, E. (1991). Group Decision Making Under Stress. Journal of Applied Psychology, 76(3), 473-478. 
Economics, F. (2014). Exploring the costs of unsafe care in the NHS. Retrieved from https://www.frontier-economics.com/documents/2014/10/exploring-the-costs-ofunsafe-care-in-the-nhs-frontier-report-2-2-2-2.pdf

Edmondson. (1996). Learning from Mistakes is Easier Said Than Done: Group and Organizational Influences on the Detection and Correction of Human Error. The Journal of Applied Behavioral Science, 32(1), 5-28. doi:10.1177/0021886396321001

Edmondson. (1999). Psychological safety and learning behavior in work teams. Administrative Science Quarterly, 44(2), 350-383. doi:Doi 10.2307/2666999

Eifler, S. (2007). Evaluating the Validity of Self-Reported Deviant Behavior Using Vignette Analyses. Quality \& Quantity, 4l(2), 303-318. doi:10.1007/s11135-0079093-3

Fairchild, J., \& Hunter, S. T. (2014). "We've Got Creative Differences": The Effects of Task Conflict and Participative Safety on Team Creative Performance. The Journal of Creative Behavior, 48(1), 64-87. doi:10.1002/jocb.41

Faul, F., Erdfelder, E., Lang, A.-G., \& Buchner, A. (2007). G*Power 3: A flexible statistical power analysis program for the social, behavioral, and biomedical sciences. Behavior Research Methods, 39(2), 175-191. doi:10.3758/bf03193146

Fein, S., Hilborne, L., Kagawa-Singer, M., Spiritus, E., Keenan, C., Seymann, G., . . . Wenger, N. (2005). Advances in Patient Safety: From Research to Implementation. In K. Henriksen, J. Battles, \& E. Marks (Eds.), Concepts and Methodology (Vol. 2). Rockville, MD: Agency for Healthcare Research and Quality (US).

Firth-Cozens, J. (2002). Barriers to incident reporting. Qual Saf Health Care, 11(1), 7. Retrieved from https://www.ncbi.nlm.nih.gov/pubmed/12120590

Fishbein, M., \& Ajzen, I. (2010). Predicting and changing behavior: The reasoned action approach. New York, NY, US: Psychology Press. 
Fisher, R. J. (1993). Social Desirability Bias and the Validity of Indirect Questioning. Journal of Consumer Research, 20(2), 303-315. doi:10.1086/209351

Fogarty, G. J., \& Shaw, A. (2010). Safety climate and the Theory of Planned Behavior: Towards the prediction of unsafe behavior. Accident Analysis \& Prevention, 42(5), 1455-1459. doi:https://doi.org/10.1016/j.aap.2009.08.008

Francis, J., Eccles, M. P., Johnston, M., Walker, A. E., Grimshaw, J. M., Foy, R., . . Bonetti, D. (2004). Constructing questionnaires based on the theory of planned behaviour: A manual for health services researchers. In. Newcastle upon Tyne, UK: Centre for Health Services Research, University of Newcastle upon Tyne.

Frith, C. (2013). The psychology of volition. Experimental Brain Research, 229(3), 289299. doi:10.1007/s00221-013-3407-6

Gaba, D. M., Singer, S. J., Sinaiko, A. D., Bowen, J. D., \& Ciavarelli, A. P. (2003). Differences in safety climate between hospital personnel and naval aviators. Human Factors, 45(2), 173-185. Retrieved from $<$ Go to ISI>://WOS:000185151600001

Garcia, J., \& Gustavson, A. R. (2000). The science of self-report: Implications for research and practice. Mahwah, NJ, US: Lawrence Erlbaum Associates Publishers.

Gavaza, P., Brown, C. M., Lawson, K. A., Rascati, K. L., Wilson, J. P., \& Steinhardt, M. (2011). Influence of attitudes on pharmacists' intention to report serious adverse drug events to the Food and Drug Administration. Br J Clin Pharmacol, 72(1), 143-152. doi:10.1111/j.1365-2125.2011.03944.x

Ghasemi, A., \& Zahediasl, S. (2012). Normality tests for statistical analysis: a guide for non-statisticians. International journal of endocrinology and metabolism, 10(2), 486-489. doi:10.5812/ijem.3505

Greene, J. C., Caracelli, V. J., \& Graham, W. F. (1989). Toward a Conceptual Framework for Mixed-Method Evaluation Designs. Educational Evaluation and Policy Analysis, 11(3), 255-274. doi:10.2307/1163620 
Greenfield, L. J. (1999). Doctors and Nurses: A Troubled Partnership. Annals of Surgery, 230(3), 279-279. Retrieved from http://www.ncbi.nlm.nih.gov/pmc/articles/PMC1420873/

Grober, E. D., \& Bohnen, J. M. (2005). Defining medical error. Can J Surg, 48(1), 39-44. Retrieved from https://www.ncbi.nlm.nih.gov/pubmed/15757035

Gunn, I. P. (2000). Patient safety and human error: the big picture. CRNA, 11(1), 41-48. Retrieved from https://www.ncbi.nlm.nih.gov/pubmed/11271037

Halasyamani, L., Kripalani, S., Coleman, E., Schnipper, J., van Walraven, C., Nagamine, J., . . Manning, D. (2006). Transition of care for hospitalized elderly patients-development of a discharge checklist for hospitalists. J Hosp Med, 1(6), 354-360. doi: $10.1002 / \mathrm{jhm} .129$

Hamilton, K., Kirkpatrick, A., Rebar, A., White, K. M., \& Hagger, M. S. (2017). Protecting young children against skin cancer: Parental beliefs, roles, and regret. Psychooncology. doi:10.1002/pon.4434

Hanlon, C., Sheedy, K., Kniffin, T., \& Rosenthal, J. (2015). 2014 Guide to State Adverse Event Reporting Systems. Retrieved from http://www.nashp.org/sites/default/files/2014_Guide to_State_Adverse_Event_R eporting_Systems.pdf

Hartnell, N., MacKinnon, N., Sketris, I., \& Fleming, M. (2012). Identifying, understanding and overcoming barriers to medication error reporting in hospitals: a focus group study. BMJ Qual Saf, 21(5), 361-368. doi:10.1136/bmjqs-2011000299

Hayward, R. A., Asch, S. M., Hogan, M. M., \& et al. (2005). Sins of omission. Getting too little medical care may be the greatest threat to patient safety. J Gen Intern Med, 20, 686-691.

Henneman, E. A., \& Gawlinski, A. (2004). A "near-miss" model for describing the nurse's role in the recovery of medical errors. J Prof Nurs, 20(3), 196-201. 
Heron, M. (2019). Deaths: Leading causes for 2017. National Vital Statistics Reports. Retrieved from Hyattsville, MD:

Hocking, J. S., Lim, M. S., Read, T., \& Hellard, M. (2006). Postal surveys of physicians gave superior response rates over telephone interviews in a randomized trial. $J$ Clin Epidemiol, 59(5), 521-524. doi:10.1016/j.jclinepi.2005.10.009

Holden, R., \& Karsh, B.-T. (2007). A Review of Medical Error Reporting System Design Considerations and a Proposed Cross-Level Systems Research Framework (Vol. 49).

Horwitz, L. I., Krumholz, H. M., Green, M. L., \& Huot, S. J. (2006). Transfers of patient care between house staff on internal medicine wards: a national survey. Arch Intern Med, 166(11), 1173-1177. doi:10.1001/archinte.166.11.1173

Hung, C. C., Chu, T. P., Lee, B. O., \& Hsiao, C. C. (2016). Nurses' attitude and intention of medication administration error reporting. J Clin Nurs, 25(3-4), 445-453. doi:10.1111/jocn.13071

IBM Corp. SPSS. (Released 2018). IBM SPSS Statistics for Windows, Version 26.0.

IOM, I. o. M. (1999). To Err Is Human: Building a Safer Health System. Washington, DC: The National Academies Press.

Ivankova, N. V., Creswell, J. W., \& Stick, S. L. (2006). Using Mixed-Methods Sequential Explanatory Design: From Theory to Practice. Field Methods, 18(1), 3-20. doi:10.1177/1525822x05282260

James, J. T. (2013). A new, evidence-based estimate of patient harms associated with hospital care. Journal of Patient Safety, 9(3), 122-128. doi:10.1097/PTS.0b013e3182948a69

Jeffe, D. B., Dunagan, W. C., Garbutt, J., Burroughs, T. E., Gallagher, T. H., Hill, P. R., . . . Fraser, V. J. (2004). Using focus groups to understand physicians' and nurses' perspectives on error reporting in hospitals. Jt Comm J Qual Saf, 30(9), 471-479. 
Jentsch, F., Barnett, J., Bowers, C. A., \& Salas, E. (1999). Who is flying this plane anyway? What mishaps tell us about crew member role assignment and air crew situation awareness. Human Factors, 41(1), 1-14.

Johnson, W. G., Brennan, T. A., Newhouse, J. P., Leape, L. L., Lawthers, A. G., Hiatt, H. H., \& Weiler, P. C. (1992). The economic consequences of medical injuries. Implications for a no-fault insurance plan. JAMA, 267(18), 2487-2492. Retrieved from https://www.ncbi.nlm.nih.gov/pubmed/1573726

Jones, T. V., Gerrity, M. S., \& Earp, J. (1990). Written case simulations: do they predict physicians' behavior? J Clin Epidemiol, 43(8), 805-815.

Kaissi, A. (2008). The role of nursing in bridging the gap between managers and physicians in hospitals. Health Care Manag (Frederick), 27(2), 113-117. doi:10.1097/01.HCM.0000285041.34913.b2

Kaldjian, L. C., Jones, E. W., Wu, B. J., Forman-Hoffman, V. L., Levi, B. H., \& Rosenthal, G. E. (2008). Reporting medical errors to improve patient safety: A survey of physicians in teaching hospitals. Archives of Internal Medicine, 168(1), 40-46. doi:10.1001/archinternmed.2007.12

Kaner, E. F., Haighton, C. A., \& McAvoy, B. R. (1998). 'So much post, so busy with practice--so, no time!': a telephone survey of general practitioners' reasons for not participating in postal questionnaire surveys. Br J Gen Pract, 48(428), 1067-1069.

Kanes, C. (2010). Elaborating Professionalism: Studies in practice and theory (Vol. 5): Springer Science \& Business Media.

Kapur, N., Parand, A., Soukup, T., Reader, T., \& Sevdalis, N. (2016). Aviation and healthcare: a comparative review with implications for patient safety. JRSM Open, 7(1), 2054270415616548. doi:10.1177/2054270415616548

Karsh, B. T., Holden, R. J., Alper, S. J., \& Or, C. K. (2006). A human factors engineering paradigm for patient safety: designing to support the performance of the healthcare professional. Qual Saf Health Care, 15 Suppl 1, i59-65. doi:10.1136/qshc.2005.015974 
Kathiresan, J., \& Patro, B. K. (2013). Case vignette: a promising complement to clinical case presentations in teaching. Educ Health (Abingdon), 26(1), 21-24. doi:10.4103/1357-6283.112796

Kilduff, G. J., Willer, R., \& Anderson, C. (2016). Hierarchy and Its Discontents: Status Disagreement Leads to Withdrawal of Contribution and Lower Group Performance. Organization Science, 27(2), 373-390. doi:10.1287/orsc.2016.1058

Kingston, M. J., Evans, S. M., Smith, B. J., \& Berry, J. G. (2004). Attitudes of doctors and nurses towards incident reporting: a qualitative analysis. Med J Aust, 181(1), 36-39.

Kronman, A. C., Paasche-Orlow, M., \& Orlander, J. D. (2012). Factors associated with disclosure of medical errors by housestaff. BMJ Qual Saf, 21(4), 271-278. doi:10.1136/bmjqs-2011-000084

Lawton, R., \& Parker, D. (2002). Barriers to incident reporting in a healthcare system. Qual Saf Health Care, 11(1), 15-18.

Leape, L. L. (1994). Error in medicine. JAMA, 272(23), 1851-1857. Retrieved from https://www.ncbi.nlm.nih.gov/pubmed/7503827

Leape, L. L. (2002). Reporting of adverse events. N Engl J Med, 347(20), 1633-1638. doi:10.1056/NEJMNEJMhpr011493

Leape, L. L., \& Berwick, D. M. (2005). Five years after To Err Is Human: what have we learned? JAMA, 293(19), 2384-2390. doi:10.1001/jama.293.19.2384

Leape, L. L., Lawthers, A. G., Brennan, T. A., \& Johnson, W. G. (1993). Preventing medical injury. QRB Qual Rev Bull, 19(5), 144-149. Retrieved from https://www.ncbi.nlm.nih.gov/pubmed/8332330

Lee, Y. H., Yang, C. C., \& Chen, T. T. (2016). Barriers to incident-reporting behavior among nursing staff: A study based on the theory of planned behavior. Journal of Management \& Organization, 22(1), 1-18. doi:10.1017/jmo.2015.8 
Leonard, M., Graham, S., \& Bonacum, D. (2004). The human factor: the critical importance of effective teamwork and communication in providing safe care. Quality \& safety in health care, 13(Suppl 1), i85-i90. doi:10.1136/qshc.2004.010033

Levinson, D. (2010). Adverse Events in Hospitals: National Incidence Among Medicare Beneficiaries. (OEI-06-09-00090).

Levinson, D. (2012). Hosital Incident Reporting Systems Do Not Capture Most Patient Harm (OEI-06-09-00091 ). Retrieved from https://oig.hhs.gov/oei/reports/oei-0609-00091.asp:

Livorsi, D., Comer, A. R., Matthias, M. S., Perencevich, E. N., \& Bair, M. J. (2016). Barriers to guideline-concordant antibiotic use among inpatient physicians: A case vignette qualitative study. J Hosp Med, 11(3), 174-180. doi:10.1002/jhm.2495

Lloret Irles, D., Morell-Gomis, R., Laguia, A., \& Moriano, J. A. (2017). Design and validation of a Cannabis Use Intention Questionnaire (CUIQ) for adolescents. Adicciones, 0(0), 865. doi:10.20882/adicciones.865

Lyndon, A. (2006). Communication and Teamwork in Patient Care: How Much Can We Learn From Aviation? Journal of Obstetric, Gynecologic, \& Neonatal Nursing, 35(4), 538-546. doi:10.1111/j.1552-6909.2006.00074.x

Lyndon, A., Sexton, J. B., Simpson, K. R., Rosenstein, A., Lee, K. A., \& Wachter, R. M. (2012). Predictors of likelihood of speaking up about safety concerns in labour and delivery. BMJ Quality \&amp; Safety, 21(9), 791-799. doi:10.1136/bmjqs2010-050211

Makary, M. A., \& Daniel, M. (2016). Medical error-the third leading cause of death in the US. $B M J, 353, \mathrm{i} 2139$. doi:10.1136/bmj.i2139

Martinez, V., Attal, N., Vanzo, B., Vicaut, E., Gautier, J. M., Bouhassira, D., \& LanteriMinet, M. (2014). Adherence of French GPs to chronic neuropathic pain clinical guidelines: results of a cross-sectional, randomized, "e" case-vignette survey. PLoS One, 9(4), e93855. doi:10.1371/journal.pone.0093855 
Nembhard, I. M., \& Edmondson, A. C. (2006). Making It Safe: The Effects of Leader Inclusiveness and Professional Status on Psychological Safety and Improvement Efforts in Health Care Teams. Journal of Organizational Behavior, 27(7), 941966. Retrieved from http://www.jstor.org/stable/4093878

O'Neil, A. C., Petersen, L. A., Cook, E. F., Bates, D. W., Lee, T. H., \& Brennan, T. A. (1993). Physician reporting compared with medical-record review to identify adverse medical events. Ann Intern Med, 119(5), 370-376. Retrieved from https://www.ncbi.nlm.nih.gov/pubmed/8338290

Occupational Safety and Health Administration (OSHA). (2017). Retrieved from https://www.osha.gov/data/commonstats

Okuyama, A., Wagner, C., \& Bijnen, B. (2014). Speaking up for patient safety by hospital-based health care professionals: a literature review. BMC Health Serv Res, 14, 61. doi:10.1186/1472-6963-14-61

Pagliari, C., \& Grimshaw, J. (2002). Impact of group structure and process on multidisciplinary evidence-based guideline development: an observational study. J Eval Clin Pract, 8(2), 145-153.

Parkinson, B., \& Manstead, A. S. R. (1993). Making sense of emotion in stories and social life. Cognition and Emotion, 7(3-4), 295-323. doi:10.1080/02699939308409191

Patankar, M., \& Sabin, E. (2008). Safety Culture Transformation Project Report. Retrieved from

Perrow, C. (1994). The Limits of Safety: The Enhancement of a Theory of Accidents. Journal of Contingencies and Crisis Management, 2(4), 212-220. doi:10.1111/j.1468-5973.1994.tb00046.x

Pfeiffer, Y., Manser, T., \& Wehner, T. (2010). Conceptualising barriers to incident reporting: a psychological framework. Qual Saf Health Care, 19(6), e60. doi:10.1136/qshc.2008.030445 
Poorolajal, J., Rezaie, S., \& Aghighi, N. (2015). Barriers to Medical Error Reporting. Int J Prev Med, 6, 97. doi:10.4103/2008-7802.166680

Probst, T. M., \& Estrada, A. X. (2010). Accident under-reporting among employees: Testing the moderating influence of psychological safety climate and supervisor enforcement of safety practices. Accident Analysis and Prevention, 42(5), 14381444. doi:10.1016/j.aap.2009.06.027

Reason, J. (1990). The contribution of latent human failures to the breakdown of complex systems. Philos Trans R Soc Lond B Biol Sci, 327(1241), 475-484. Retrieved from https://www.ncbi.nlm.nih.gov/pubmed/1970893

Record, R. A., Harrington, N. G., Helme, D. W., \& Savage, M. W. Using the Theory of Planned Behavior to Guide Focus Group Development of Messages Aimed at Increasing Compliance With a Tobacco-Free Policy. American Journal of Health Promotion, O(0), 0890117116687467. doi:10.1177/0890117116687467

Rivis, A., \& Sheeran, P. (2003). Descriptive norms as an additional predictor in the theory of planned behaviour: A meta-analysis. Current Psychology, 22(3), 218233. doi:10.1007/s12144-003-1018-2

Robertson, J., Walkom, E. J., \& McGettigan, P. (2005). Response rates and representativeness: a lottery incentive improves physician survey return rates. Pharmacoepidemiol Drug Saf, 14(8), 571-577. doi:10.1002/pds.1126

Robinson, A. R., Hohmann, K. B., Rifkin, J. I., Topp, D., Gilroy, C. M., Pickard, J. A., \& Anderson, R. J. (2002). Physician and public opinions on quality of health care and the problem of medical errors. Archives of Internal Medicine, 162(19), 21862190. doi:10.1001/archinte.162.19.2186

Rochlin, G. (1999). Safe Operation as a Social Construct (Vol. 42).

Rowin, E. J., Lucier, D., Pauker, S. G., Kumar, S., Chen, J., \& Salem, D. N. (2008). Does error and adverse event reporting by physicians and nurses differ? Jt Comm $J$ Qual Patient Saf, 34(9), 537-545. 
Sagan, S. (1993). The Origins of Accidents. In The Limits of Safety: Organizations, Accidents, and Nuclear Weapons. Princeton: Princeton University Press.

Salas, E., Burke, C. S., Bowers, C. A., \& Wilson, K. A. (2001). Team training in the skies: does crew resource management (CRM) training work? Human Factors, 43(4), 641-674. doi:10.1518/001872001775870386

Salas, E., Wilson, K. A., Burke, C. S., \& Wightman, D. C. (2006). Does crew resource management training work? An update, an extension, and some critical needs. Human Factors, 48(2), 392-412. doi:10.1518/001872006777724444

Schein, E. H., \& Bennis, W. G. (1965). Personal and Organizational Change Through Group Methods: The Laboratory Approach. In Canadian Journal of Counselling and Psychotherapy (Vol. 17).

Schroder, C., Behnke, M., Gastmeier, P., Schwab, F., \& Geffers, C. (2015). Case vignettes to evaluate the accuracy of identifying healthcare-associated infections by surveillance persons. J Hosp Infect, 90(4), 322-326.

doi:10.1016/j.jhin.2015.01.014

Sexton, Thomas, E., \& Helmreich, R. (2000). Error, stress, and teamwork in medicine and aviation: cross sectional surveys. BMJ, 320(7237), 745-749. doi:10.1136/bmj.320.7237.745

Sexton, B., Marsch, S., Helmreich, R., Betzenboerfer, D., Kocher, T., Scheidegger, D., \& Team, T. (1998). Jumpseating in the Operating Room. In L. C. Henson \& A. C. Lee (Eds.), Simulators in Anesthesiology Education. Boston, MA: Springer.

Shojania, K. G., Duncan, B. W., McDonald, K. M., \& Wachter, R. M. (2001). Making Health Care Safer: A Critical Analysis of Patient Safety Practices (43). Retrieved from

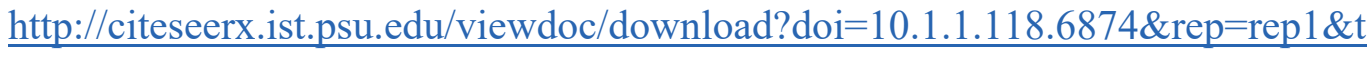
ype $=p d f$

Simpson, K. R., \& Knox, G. E. (2003). Adverse perinatal outcomes. Recognizing, understanding \& preventing common accidents. AWHONN Lifelines, 7(3), 224235. 
Sorra, J., Gray, L., Streagle, S., Famolaro, T., Yount, N., \& Behm, J. (2016). AHRQ Hospital Survey on Patient Safety Culture: User's Guide. (Publication No. 150049-EF (Replaces 04-0041)). Rockville, MD: Westat Retrieved from https://www.ahrq.gov/sites/default/files/wysiwyg/professionals/quality-patientsafety/patientsafetyculture/hospital/userguide/hospcult.pdf

Sundar, E., Sundar, S., Pawlowski, J., Blum, R., Feinstein, D., \& Pratt, S. (2007). Crew resource management and team training. Anesthesiol Clin, 25(2), 283-300. doi:10.1016/j.anclin.2007.03.011

Suresh, G., Horbar, J. D., Plsek, P., Gray, J., Edwards, W. H., Shiono, P. H., . . Goldmann, D. (2004). Voluntary anonymous reporting of medical errors for neonatal intensive care. Pediatrics, 113(6), 1609-1618.

Tabachnick, B. G., \& Fidell, L. S. (2007). Using multivariate statistics (5th ed. ed.). Boston: Pearson/Allyn \& Bacon.

Tajfel, H., \& Turner, J. C. (2004). The Social Identity Theory of Intergroup Behavior. New York, NY, US: Psychology Press.

Tamuz, M., \& Harrison, M. I. (2006). Improving Patient Safety in Hospitals: Contributions of High-Reliability Theory and Normal Accident Theory. Health Services Research, 41(4p2), 1654-1676. doi:10.1111/j.1475-6773.2006.00570.x

Tashakkori, A., \& Teddlie, C. (1998). Mixed methodology: Combining qualitative and quantitative approaches. Thousand Oaks, CA, US: Sage Publications, Inc.

The Joint Commission. (2020). Retrieved from https://www.jointcommission.org/en/

Thomas, E. J., Studdert, D. M., Burstin, H. R., Orav, E. J., Zeena, T., Williams, E. J., . . . Brennan, T. A. (2000). Incidence and types of adverse events and negligent care in Utah and Colorado. Med Care, 38(3), 261-271. Retrieved from https://www.ncbi.nlm.nih.gov/pubmed/10718351

Thomas, E. J., Studdert, D. M., Newhouse, J. P., Zbar, B. I., Howard, K. M., Williams, E. J., \& Brennan, T. A. (1999). Costs of medical injuries in Utah and Colorado. 
Inquiry, 36(3), 255-264. Retrieved from

https://www.ncbi.nlm.nih.gov/pubmed/10570659

Tucker, A. L., Singer, S. J., Hayes, J. E., \& Falwell, A. (2008). Front-Line Staff Perspectives on Opportunities for Improving the Safety and Efficiency of Hospital Work Systems. Health Services Research, 43(5 Pt 2), 1807-1829. doi:10.1111/j.1475-6773.2008.00868.x

Tuttle, D., Holloway, R., Baird, T., Sheehan, B., \& Skelton, W. K. (2004). Electronic reporting to improve patient safety. Qual Saf Health Care, 13(4), 281-286. doi:10.1136/qhc.13.4.281

Tyler, T., \& Lind, E. (1992). A Relational Model of Authority in Groups (Vol. 25).

Uribe, C. L., Schweikhart, S. B., Pathak, D. S., Dow, M., \& Marsh, G. B. (2002). Perceived barriers to medical-error reporting: an exploratory investigation. $J$ Healthc Manag, 47(4), 263-279. Retrieved from https://www.ncbi.nlm.nih.gov/pubmed/12221747

Van Den Bos, J., Rustagi, K., Gray, T., Halford, M., Ziemkiewicz, E., \& Shreve, J. (2011). The $\$ 17.1$ billion problem: the annual cost of measurable medical errors. Health Aff (Millwood), 30(4), 596-603. doi:10.1377/hlthaff.2011.0084

Van den Broeck, J., Cunningham, S. A., Eeckels, R., \& Herbst, K. (2005). Data cleaning: detecting, diagnosing, and editing data abnormalities. PLoS medicine, 2(10), e267-e267. doi:10.1371/journal.pmed.0020267

Vincent, C., Neale, G., \& Woloshynowych, M. (2001). Adverse events in British hospitals: preliminary retrospective record review. BMJ, 322(7285), 517-519. Retrieved from https://www.ncbi.nlm.nih.gov/pubmed/11230064

Wakefield, D. S., Wakefield, B. J., Uden-Holman, T., Borders, T., Blegen, M., \& Vaughn, T. (1999). Understanding why medication administration errors may not be reported. Am J Med Qual, 14(2), 81-88. doi:10.1177/106286069901400203

Waring. (2005). Beyond blame: cultural barriers to medical incident reporting. Social 
Science \& Medicine, 60(9), 1927-1935.

doi:https://doi.org/10.1016/j.socscimed.2004.08.055

Weingart, Callanan, L. D., Ship, A. N., \& Aronson, M. D. (2001). A Physician-based Voluntary Reporting System for Adverse Events and Medical Errors. J Gen Intern Med, 16(12), 809-814. doi:doi:10.1046/j.1525-1497.2001.10231.x

Weingart, Pagovich, O., Sands, D. Z., Li, J. M., Aronson, M. D., Davis, R. B., . . Phillips, R. S. (2005). What Can Hospitalized Patients Tell Us About Adverse Events? Learning from Patient-Reported Incidents. J Gen Intern Med, 20(9), 830836. doi:10.1111/j.1525-1497.2005.0180.x

Weissman, J. S., Schneider, E. C., Weingart, S. N., Epstein, A. M., David-Kasdan, J., Feibelmann, S., . . Gatsonis, C. (2008). Comparing patient-reported hospital adverse events with medical record review: do patients know something that hospitals do not? Ann Intern Med, 149(2), 100-108.

Wetzel, A. P., Dow, A. W., \& Mazmanian, P. E. (2012). Patient safety attitudes and behaviors of graduating medical students. Eval Health Prof, 35(2), 221-238. doi: $10.1177 / 0163278711414560$

WHO, W. H. O. (2002). Quality of care: patient safety. Retrieved from

WHO, W. H. O. (2005a). WHO Draft Guidelines for Adverse Event Reporting and Learning Systems. Retrieved from Geneva, Switzerland: http://apps.who.int/iris/bitstream/10665/69797/1/WHO-EIP-SPO-QPS-05.3eng.pdf

WHO, W. H. O. (2005b). World Alliance for Patient Safety. Retrieved from Geneva, Switzerland: http://www.who.int/patientsafety/en/brochure_final.pdf

WHO, W. H. O. (2019). Patient Safety: Key Facts. Retrieved from https://www.who.int/news-room/fact-sheets/detail/patient-safety

Wiener, E., Kanki, B., \& Helmreich, R. (1993). Cockpit resource management. San Diego, CA: Academic Press. 
Williams, S. D., Phipps, D. L., \& Ashcroft, D. (2015). Examining the attitudes of hospital pharmacists to reporting medication safety incidents using the theory of planned behaviour. International Journal for Quality in Health Care, 27(4), 297-304. doi:10.1093/intqhe/mzv044

Wu, A. W. (2000). Medical error: the second victim. The doctor who makes the mistake needs help too, 320(7237), 726-727. doi:10.1136/bmj.320.7237.726

Zhao, B., \& Olivera, F. (2006). Error Reporting in Organizations. The Academy of Management Review, 31(4), 1012-1030. doi:10.2307/20159263

Zwarenstein, M., \& Bryant, W. (2000). Interventions to promote collaboration between nurses and doctors. Cochrane Database Syst Rev(2), Cd000072. doi:10.1002/14651858.cd000072 


\section{APPENDICES}

Appendix A: Institutional Review Board (IRB) approval

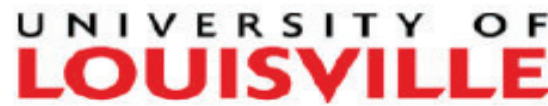

\begin{tabular}{l}
\hline DATE: \\
TO: \\
FROM: \\
IRB NUMBER: \\
STUDY TITLE: \\
REFERENCE \#: \\
IRB STAFF \\
CONTACT:
\end{tabular}

August 30, 2018

Whitney T Rogers

18.0092

654767
The University of Louisville Institutional Review Board

Predicting Medical Error Reporting Behavior: The Influence of Psychological Safety and the Theory of Planned Behavior

Barbara Dearinger 852-5987 badear01@louisville.edu

This study was reviewed on $08 / 30 / 2018$ by the Chair/Vice Chair of the Institutional Review Board and approved through the Expedited Review Procedure, according to 45 CFR 46.110 (b), since this study falls under Category 7: Research on individual or group characteristics or behavior (including, but not limited to, research on perception, cognition, motivation, identity, language, communication, cultural beliefs or practices, and social behavior) or research employing survey, interview, oral history, focus group, program evaluation, human factors evaluation, or quality assurance methodologies. This study now has final IRB approval from 08/30/2018 through 08/29/2019.

This study was also approved through 45 CFR 46.116 (C), which means that an IRB may waive the requirement for the investigator to obtain a signed informed consent form for some or all subjects if it finds either:

- That the only record linking the subject and the research would be the consent document and the principal risk would be potential harm resulting from a breach of confidentiality. Each subject will be asked whether the subject wants documentation linking the subject with the research, and the subject's wishes will govern; or

- That the research presents no more than minimal risk of harm to subjects and involves no procedures for which written consent is normally required outside of the research context.

The following items have been approved:

\begin{tabular}{|l|l|l|}
\hline Title & Version Date & Outcome \\
\hline Protocol & $08 / 16 / 2018$ & Approved \\
\hline Introductory Email Letter & $08 / 09 / 2018$ & Approved \\
\hline Survey Instrument & $08 / 09 / 2018$ & Approved \\
\hline Preamble & $08 / 17 / 2018$ & Approved \\
\hline
\end{tabular}

For guidance on using iRIS, including finding your approved stamped documents, please follow the instructions at https://louisville.edu/research/humansubjects/iRISSubmissionManual.pdf 


\section{U NIVERSITY OF LOUISVILLE}

Human Subjects Protection Program Office

MedCenter One - Suite 200

$501 \mathrm{E}$. Broadway

Louisville, KY 40202-1798

Office: 502.852 .5188 Fax: 502.852 .2164

$\begin{array}{ll}\text { DATE: } & \text { August } 14,2019 \\ \text { TO: } & \text { Whitney T Rogers } \\ \text { IRB NUMBER: } & 18.0092 \\ \text { STUDY TITLE: } & \text { Predicting Medical Error Reporting Behavior: The Influence of Psychological } \\ & \text { Safety and the Theory of Planned Behavior } \\ \text { REFERENCE \#: } & 689557 \\ \text { REVIEW DATE: } & 8 / 13 / 2019 \\ \text { IRB STAFF CONTACT: } & \text { Barbara Dearinger } 852-5987 \text { badear01@louisville.edu }\end{array}$

The continuation request for this study was reviewed by the Chair/Vice-Chair of the Institutional Review Board (IRB) through the expedited review procedure, according to 45 CFR 46.110 and 21 CFR 56.110 since this study falls under Category 7: Research on individual or group characteristics or behavior (including, but not limited to, research on perception, cognition, motivation, identity, language, communication, cultural beliefs or practices, and social behavior) or research employing survey, interview, oral history, focus group, program evaluation, human factors evaluation, or quality assurance methodologies. The study now has continued committee approval from $08 / 30 / 2019$ through $08 / 29 / 2020$.

The following items were reviewed and approved:

\begin{tabular}{|l|l|l|}
\hline Title & Version Date & Outcome \\
\hline Protocol & $08 / 16 / 2018$ & Approved \\
\hline Introductory Email Letter & $08 / 09 / 2018$ & Approved \\
\hline Survey Instrument & $08 / 09 / 2018$ & Approved \\
\hline
\end{tabular}

Continuation Review Requirements

You are responsible for submitting a continuation review 30 days prior to the expiration date of your research study. Investigators who allow their study approval to expire have committed significant non-compliance with federal regulations. Such lapses may require reporting to federal agencies, a program audit by compliance auditors to ensure that subjects were not enrolled during the expired period, and may lead to findings of serious and continuing noncompliance if expiration were to occur a second time.

This action will be reported promptly to the IRB at a scheduled full Board meeting.

If you have any questions, please contact the IRB analyst listed above or the Human Subjects Protection Program office at hsppofc@louisville.edu.

We value your feedback. Please let us know how you think we are doing: https://www.surveymonkey.com/r/CCLHXRP

Sincerely,

Pta $m$ ansach

Peter M. Quesada, Ph.D., Vice Chair

Biomedical Institutional Review Board 
Site Approval

If this study will take place at an affiliated research institution, such as KentuckyOne Health, Norton Healthcare or University of Louisville Hospital, permission to use the site of the affiliated institution is necessary before the research may begin. If this study will take place outside of the University of Louisville Campuses, permission from the organization must be obtained before the research may begin (e.g. Jefferson County Public Schools). Failure to obtain this permission may result in a delay in the start of your research.

\section{Privacy \& Encryption Statement}

The University of Louisville's Privacy and Encryption Policy requires such information as identifiable medical and health records: credit card, bank account and other personal financial information; social security numbers; proprietary research data; dates of birth (when combined with name, address and/or phone numbers) to be encrypted. For additional information:

http://security.louisville.edu/PolStds/ISO/PS018.htm.

\section{Implementation of Changes to Previously Approved Research}

Prior to the implementation of any changes in the approved research, the investigator will submit any modifications to the IRB and await approval before implementing the changes, unless the change is being made to ensure the safety and welfare of the subjects enrolled in the research. If such occurs, a Protocol Deviation/Violation should be submitted within five days of the occurrence indicating what safety measures were taken, along with an amendment to revise the protocol.

Unanticipated Problems Involving Risks to Subjects or Others (UPIRTSOs)

In general, these may include any incident, experience, or outcome, which has been associated with an unexpected event(s), related or possibly related to participation in the research, and suggests that the research places subjects or others at a greater risk of harm than was previously known or suspected. UPIRTSOs may or may not require suspension of the research. Each incident is evaluated on a case by case basis to make this determination. The IRB may require remedial action or education as deemed necessary for the investigator or any other key personnel. The investigator is responsible for reporting UPIRTSOs to the IRB within 5 working days. Use the UPIRTSO form located within the iRIS system to report any UPIRTSOs.

Continuation Review Requirements

You are responsible for submitting a continuation review 30 days prior to the expiration date of your research study. Investigators who allow their study approval to expire have committed significant noncompliance with federal regulations. Such lapses may require reporting to federal agencies, a program audit by compliance auditors to ensure that subjects were not enrolled during the expired period, and may lead to findings of serious and continuing non-compliance if expiration were to occur a second time.

Full Accreditation since June 2005 by the Association for the Accreditation of Human Research Protection Programs, Inc.

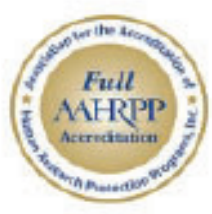


The committee will be advised of this action at a regularly scheduled meeting.

If you have any questions, please contact the IRB analyst listed above or the Human Subjects Protection Program office at hsppofc@louisville.edu.

Sincerely,

\section{Prefopadnuch}

Paula Radmacher, Ph.D., Vice Chair

Biomedical Institutional Review Board

We value your feedback. Please let us know how you think we are doing: httos:/Wwww.surveymonkey.com/r/CCLHXRP

Full Accreditation since June 2005 by the Association for the Accreditation of Human Research Protection Programs, Inc.

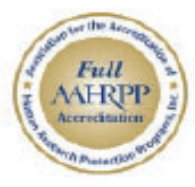

117 
Appendix B: Survey Instrument

$7 / 1 / 2019$

Qualtrics Survey Software

\section{Preamble}

\section{ynuvessiry of LOUISVILLE. \\ SCHOOL OF PUBLIC HEALTH \\ \& INFORMATION SCIENCES}

Welcome to the Patient Safety Survey!

Date $10 / 17 / 18$

You are being invited to participate in a research study by answering the attached survey. You will be asked questions about patient safety. There are no known risks for your participation in this research study. The information collected may not benefit you directly. The information learned in this study may be helpful to others. The information you provide will be used to better understand issues related to patient safety in healthcare.

No identifying information will be collected and your participation will be anonymous. Your completed survey will be stored at the School of Public Health \& Information Sciences. The survey will take approximately 15 minutes to complete. 
Individuals from the School of Public Health \& Information Sciences, the Institutional Review Board (IRB), the Human Subjects Protection Program Office (HSPPO), and other regulatory agencies may inspect these records. In all other respects, however, the data will be held in confidence to the extent permitted by law. Should the data be published, your identity will not be disclosed.

Taking part in this study is voluntary. By completing this survey you agree to take part in this research study. You do not have to answer any questions that make you feel uncomfortable. You may choose not to take part at all. If you decide to be in this study you may stop taking part at any time. If you decide not to be in this study or if you stop taking part at any time, you will not lose any benefits for which you may qualify.

If you have any questions, concerns, or complaints about the research study, please contact: Whitney Rogers, MA, MPH at 502-852-3045 or Dr. Scott LaJoie at 502-852-1879.

If you have any questions about your rights as a research subject, you may call the Human Subjects Protection Program Office at (502) 852-5188. You can discuss any questions about your rights as a research subject, in private, with a member of the Institutional Review Board (IRB). You may also call this number if you have other questions about the research, and you cannot reach 
the research staff, or want to talk to someone else. The IRB is an independent committee made up of people from the University community, staff of the institutions, as well as people from the community not connected with these institutions. The IRB has reviewed this research study.

If you have concerns or complaints about the research or research staff and you do not wish to give your name, you may call $1-877-852-1167$. This is a 24 hour hot line answered by people who do not work at the University of Louisville.

Sincerely,

Whitney T. Rogers, MA, MPH

Principal Investigator

A. Scott LaJoie, PhD

Co-Investigator 


\begin{tabular}{|c|c|c|}
\hline \multicolumn{3}{|c|}{$\begin{array}{l}\text { Reporting Medical Errors } \\
\text { Survey Instrument }\end{array}$} \\
\hline \multicolumn{2}{|c|}{$\# Q$ 's } & Source \\
\hline \multirow[t]{5}{*}{7} & Background/Demographics & \\
\hline & $\begin{array}{l}\text { Gender } \\
\text { a) Male } \\
\text { b) Female } \\
\text { c) Other }\end{array}$ & \\
\hline & Age & \\
\hline & $\begin{array}{l}\text { Race (check all that apply) } \\
\text { a) White } \\
\text { b) Black or African American } \\
\text { c) Asian } \\
\text { d) American Indian or Alaska Native } \\
\text { e) Pacific Islander or Native Hawaiian } \\
\text { f) Other }\end{array}$ & $C D C$ \\
\hline & $\begin{array}{l}\text { What is your profession/position? } \\
\text { a) Licensed Practical Nurse (LPN) } \\
\text { b) Registered Nurse } \\
\text { c) Nurse Practitioner } \\
\text { d) Physician Assistant } \\
\text { e) Attending/Staff Physician } \\
\text { f) Resident or Fellow }\end{array}$ & $\begin{array}{l}\text { AHQR } \\
\text { Section } \\
\text { H4 } \\
\text { (modified) }\end{array}$ \\
\hline
\end{tabular}




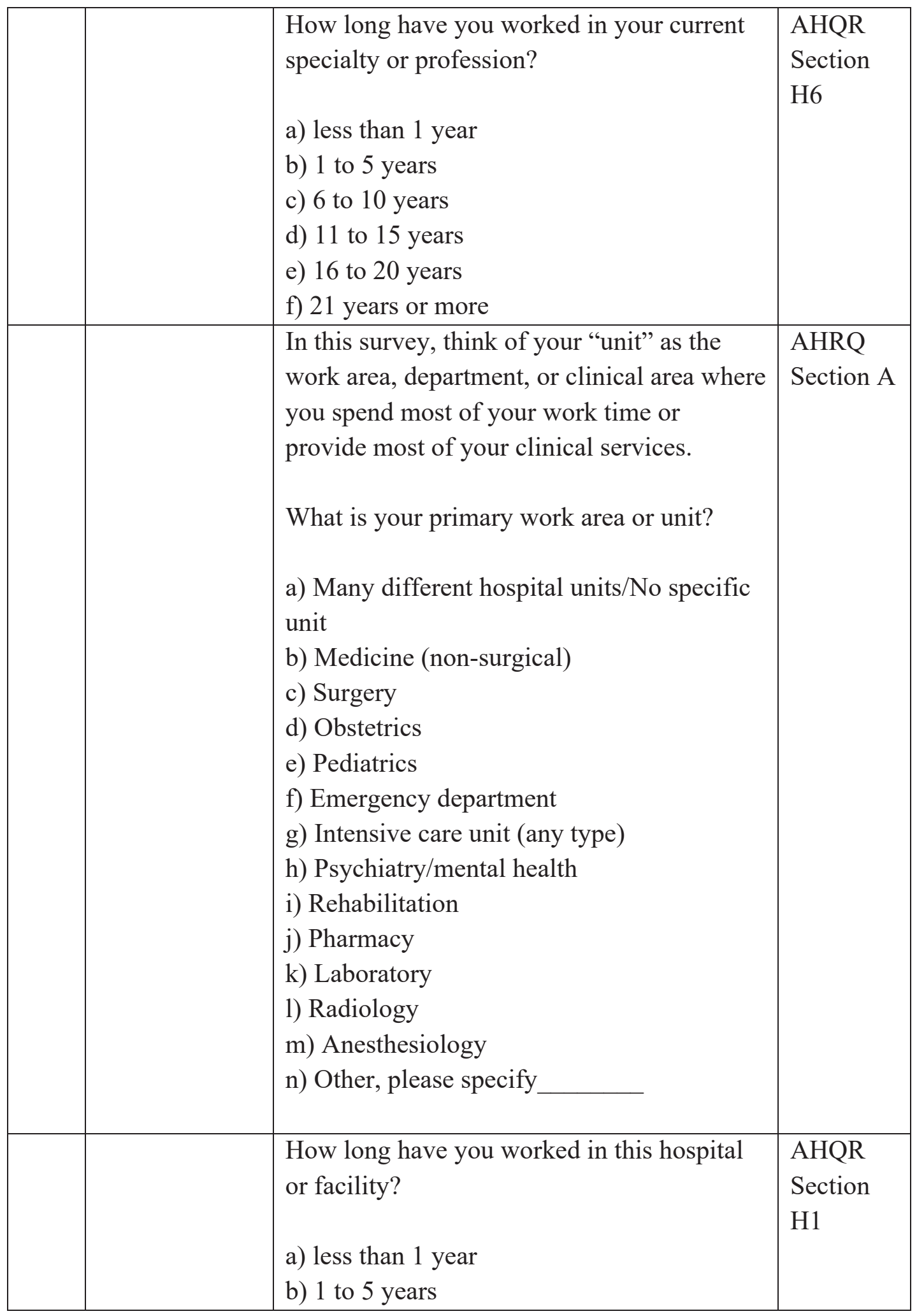




\begin{tabular}{|c|c|c|}
\hline & $\begin{array}{l}\text { c) } 6 \text { to } 10 \text { years } \\
\text { d) } 11 \text { to } 15 \text { years } \\
\text { e) } 16 \text { to } 20 \text { years } \\
\text { f) } 21 \text { years or more }\end{array}$ & \\
\hline 2 & \multicolumn{2}{|l|}{ Reporting Skills } \\
\hline & $\begin{array}{l}\text { I know how to report medical errors. } \\
\text { SD - SA ( } 5 \text { pt.) }\end{array}$ & $\begin{array}{l}\text { Kaldjian, } \\
2008\end{array}$ \\
\hline & $\begin{array}{l}\text { I know what kind of errors should be } \\
\text { reported. } \\
\text { SD - SA ( } 5 \text { pt.) }\end{array}$ & $\begin{array}{l}\text { Kaldjian, } \\
2008\end{array}$ \\
\hline
\end{tabular}

- The following questions ask about your experience with reporting medical errors that you were personally involved in or observed.

- Medical errors go by many names and can be called events, incidents, slips, near misses to name a few.

- Reporting medical errors refers to either making a formal report to an incident reporting system or to a supervisor/manager.

\begin{tabular}{|c|c|c|c|}
\hline 2 & Past Behavior & & \\
\hline & & $\begin{array}{l}\text { In the past } 12 \text { months, how many medical } \\
\text { errors or "events" have you committed or } \\
\text { observed? } \\
\text { a) None } \\
\text { b) } 1 \text { to } 2 \\
\text { c) } 3 \text { to } 5 \\
\text { d) } 6 \text { to } 10 \\
\text { e) } 11 \text { to } 20 \\
\text { f) } 21 \text { or more }\end{array}$ & $\begin{array}{l}\text { AHQR } \\
\text { Section G }\end{array}$ \\
\hline & & $\begin{array}{l}\text { In the past } 12 \text { months, how many of these } \\
\text { medical errors or "events" (that you } \\
\text { personally committed or observed) have you } \\
\text { reported to an incident reporting system or a } \\
\text { manager/supervisor? }\end{array}$ & \\
\hline
\end{tabular}




\begin{tabular}{|c|c|c|c|}
\hline & & $\begin{array}{l}\text { a) No event reports } \\
\text { b) } 1 \text { to } 2 \text { event reports } \\
\text { c) } 3 \text { to } 5 \text { event reports } \\
\text { d) } 6 \text { to } 10 \text { event reports } \\
\text { e) } 11 \text { to } 20 \text { event reports } \\
\text { f) } 21 \text { or more event reports }\end{array}$ & \\
\hline \multirow[t]{4}{*}{3} & \multicolumn{3}{|c|}{$\begin{array}{l}\text { Intention to Report Medical Errors: To what extent do you agree with } \\
\text { the following statements: }\end{array}$} \\
\hline & & $\begin{array}{l}\text { I expect that I will report medical errors } \\
\text { SD - SA ( } 5 \text { pt.) }\end{array}$ & $\begin{array}{l}\text { Francis } \\
(2004)\end{array}$ \\
\hline & & $\begin{array}{l}\text { If a medical error occurs, I want to report it. } \\
\text { SD - SA ( } 5 \text { pt.) }\end{array}$ & \\
\hline & & $\begin{array}{l}\text { I intend to report medical errors } \\
\text { SD - SA ( } 5 \text { pt.) }\end{array}$ & \\
\hline \multirow[t]{5}{*}{4} & \multicolumn{3}{|c|}{$\begin{array}{l}\text { Attitude (towards the behavior): Overall, I think reporting medical errors } \\
\text { is }\end{array}$} \\
\hline & & Good - Bad Practice (Overall evaluation) & $\begin{array}{l}\text { Francis } \\
(2004)\end{array}$ \\
\hline & & Harmful - Beneficial (Instrumental) & \\
\hline & & Pleasant (for me) - Unpleasant (Experiential) & \\
\hline & & Worthless - Useful (Instrumental) & \\
\hline \multirow[t]{4}{*}{4} & \multicolumn{3}{|c|}{$\begin{array}{l}\text { Subjective Norms: To what extent do you agree with the following } \\
\text { statements: }\end{array}$} \\
\hline & Injunctive & $\begin{array}{l}\text { Most people who are important to me think } \\
\text { that I should report medical errors } \\
\text { SD - SA ( } 5 \text { pt.) }\end{array}$ & $\begin{array}{l}\text { Ajzen; } \\
\text { Francis } \\
(2004)\end{array}$ \\
\hline & Injunctive & $\begin{array}{l}\text { It is expected of me that I report medical } \\
\text { errors } \\
\text { SD - SA ( } 5 \text { pt.) }\end{array}$ & \\
\hline & Injunctive & $\begin{array}{l}\text { I feel under social pressure to report medical } \\
\text { errors } \\
\text { SD - SA ( } 5 \text { pt.) }\end{array}$ & \\
\hline
\end{tabular}




\begin{tabular}{|c|c|c|c|}
\hline & Descriptive & $\begin{array}{l}\text { Most people like me report medical errors } \\
\text { SD - SA ( } 5 \text { pt.) }\end{array}$ & \\
\hline 4 & \multicolumn{3}{|c|}{$\begin{array}{l}\text { Perceived Behavioral Control: To what extent do you agree with the } \\
\text { following statements: }\end{array}$} \\
\hline & Self-efficacy & $\begin{array}{l}\text { I am confident that I could report a medical } \\
\text { error if I wanted to } \\
\text { SD - SA ( } 5 \text { pt.) }\end{array}$ & $\begin{array}{l}\text { Francis } \\
(2004)\end{array}$ \\
\hline & Self-efficacy & $\begin{array}{l}\text { For me to report a medical error is } \\
\text { Easy - Difficult ( } 5 \text { pt.) }\end{array}$ & \\
\hline & Controllability & $\begin{array}{l}\text { The decision to report a medical error is } \\
\text { beyond my control } \\
\text { SD - SA ( } 5 \text { pt.) }\end{array}$ & \\
\hline & Controllability & $\begin{array}{l}\text { Whether I report a medical error or not was } \\
\text { entirely up to me } \\
\text { SD - SA ( } 5 \text { pt.) }\end{array}$ & \\
\hline 7 & & Psychological Safety & \\
\hline \multicolumn{4}{|c|}{$\begin{array}{l}\text { The following questions ask about your team or unit. This refers to the people in } \\
\text { your work area, department, or clinical area where you spend most of your work } \\
\text { time or provide most of your clinical services. }\end{array}$} \\
\hline & & $\begin{array}{l}\text { If you make a mistake on my team, it is } \\
\text { often held against you } \\
\text { SD - SA ( } 5 \text { pt.) }\end{array}$ & $\begin{array}{l}\text { Edmondson } \\
(1999) \\
\text { Reverse } \\
\text { scored }\end{array}$ \\
\hline & & $\begin{array}{l}\text { Members of my team are able to bring up } \\
\text { problems and tough issues } \\
\text { SD - SA ( } 5 \text { pt.) }\end{array}$ & \\
\hline & & $\begin{array}{l}\text { People on my team sometimes reject others } \\
\text { for being different } \\
\text { SD - SA ( } 5 \text { pt.) }\end{array}$ & $\begin{array}{l}\text { Reverse } \\
\text { scored }\end{array}$ \\
\hline & & $\begin{array}{l}\text { It is safe to take a risk on my team } \\
\text { SD - SA ( } 5 \text { pt.) }\end{array}$ & \\
\hline
\end{tabular}




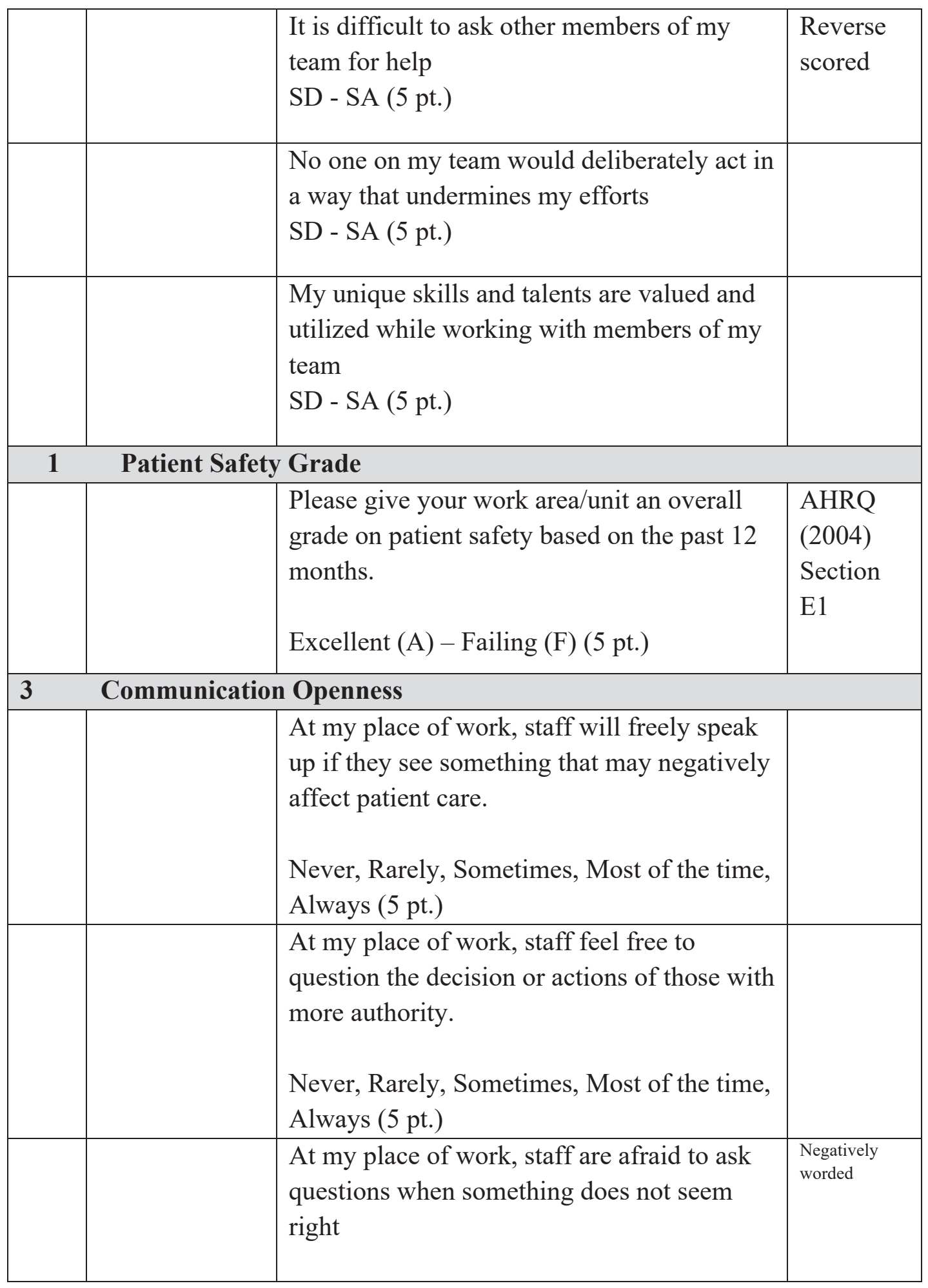




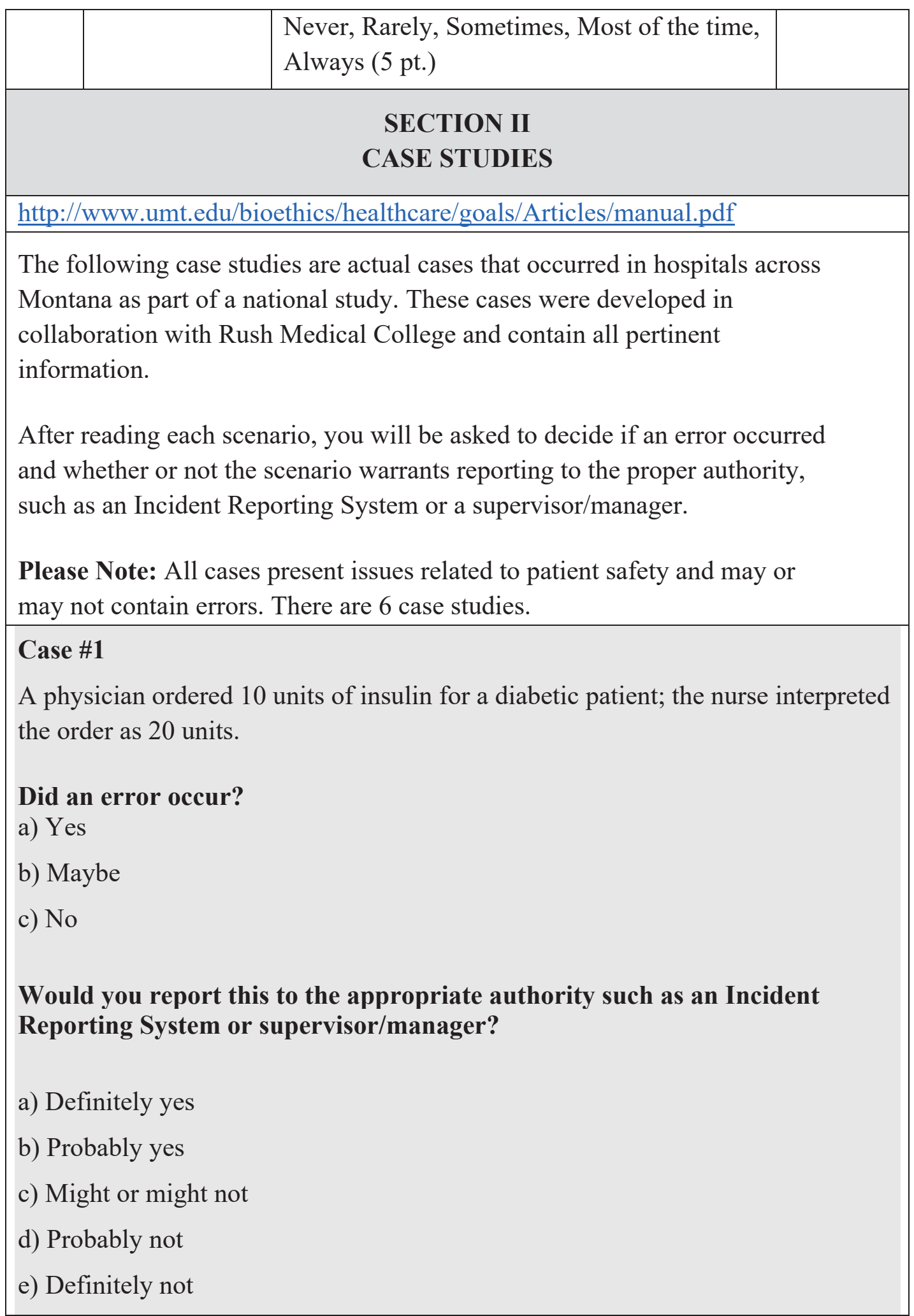


Do you think your colleagues would report this case to the appropriate authority such as an Incident Reporting System or supervisor/manager?
a) Definitely yes
b) Probably yes
c) Might or might not
d) Probably not
e) Definitely not

\section{Case \#2}

An 83-year old male was diagnosed with atrial fibrillation and was admitted to the hospital for evaluation. His heart rate was controlled, he was started on Heparin and Coumadin, and when his INR reached a value of 2.5, he was discharged on a Coumadin dose of $5 \mathrm{mg}$ /day. No follow-up lab tests were done or ordered before the patient's scheduled visit to the clinic in 3 weeks. He came to ER one day before the scheduled visit with an INR of 14.7 and pain from an expanding spontaneous hematoma of his thigh.

\section{Did an error occur?}
a) Yes
b) Maybe
c) No

Would you report this to the appropriate authority such as an Incident Reporting System or supervisor/manager?
a) Definitely yes
b) Probably yes
c) Might or might not
d) Probably not
e) Definitely not 


\section{Do you think your colleagues would report this case to the appropriate authority such as an Incident Reporting System or supervisor/manager?}
a) Definitely yes
b) Probably yes
c) Might or might not
d) Probably not
e) Definitely not

\section{Case \#3}

Mrs. Jenkins was admitted with diagnoses including diabetes, significant renal disease, hypertension, blindness, and an infection in her foot. Her physician cultured the foot wound and, after receiving the sensitivity report, ordered several antibiotics including "Gentamicin Sulfate $60 \mathrm{mg}$ IV q. 40 h." The nurse caring for Mrs. Jenkins wondered what the doctor meant by "q. 40 h" but he hadn't noted anything unusual. She assumed he meant every 4 hours and was using a common symbol, the superscript 0 , to mean hours. She also expected that the ward clerk would clarify the order since the physician was still at the nurses' station and so when she took the order off the chart and entered it on the medication sheet, the order read q. $4 \mathrm{~h}$.

The pharmacist believed the order for q. $4 \mathrm{~h}$. had been clarified with the MD. Finally, the first nurse to give the medication assumed that the prescription was filled correctly. Subsequent nurses did not go back to the original order in the chart (q. $40 \mathrm{~h}$ ) and assumed that the $\mathrm{q} 4 \mathrm{~h}$, listed on the medication sheet, was correct. Mrs. Jenkins received Gentamicin $60 \mathrm{mg}$ IV six times daily for three days, instead of every 40 hours as was intended due to her renal insufficiency. She suffered a permanent loss of approximately $90 \%$ of her hearing.

\section{Did an error occur?}
a) Yes
b) Maybe
c) No 
Would you report this to the appropriate authority such as an Incident Reporting System or supervisor/manager?
a) Definitely yes
b) Probably yes
c) Might or might not
d) Probably not
e) Definitely not

Do you think your colleagues would report this case to the appropriate authority such as an Incident Reporting System or supervisor/manager?
a) Definitely yes
b) Probably yes
c) Might or might not
d) Probably not
e) Definitely not 


\section{CASE \#4:}

An 83-year-old man has a total knee replacement and is put on Coumadin. Two days after surgery he is noted to have a delirium. Its cause is presumed to be postop and may be secondary to pain medications. Several nurses' notes reveal that he seems worse after getting Vicodin. However, he begins to clear over the following week, but occasional notes in his chart reveal that he is oriented X 2; once X3. His neurology exam is normal.

He is sent to the rehab program. Since improvement is slow, however, he is discharged from the rehab unit and re-admitted to the hospital. Two days after this admission, he falls and bumps his head. His INR is 1.5-1.9. However, a neurologist notes that he has no LOC, or head ache and his physical exam is normal. The patient is followed daily and closely by multiple providers; all say he is oriented to time, person, and place and no focal neurological deficits are noted. Several notes say: "no need for a CT scan as his exam remains normal".

Since his family members think the at the hospital environment may be impeding his recovery, they want him to go home to normal surroundings for a time. PT/OT staff do not think he should be discharged; they are concerned about the risk of a fall and believe he should be watched 24 hour/day. The medical team agrees to the family's requests and the patient is discharged.

Three days after his return home he is noted to be "dull and weak." He is taken to an ED and they find a large subdural hematoma with herniation. The patient dies.

\section{Did an error occur?}
a) Yes
b) Maybe
c) No

Would you report this to the appropriate authority such as an Incident Reporting System or supervisor/manager?
a) Definitely yes
b) Probably yes
c) Might or might not 
d) Probably not

e) Definitely not

Do you think your colleagues would report this case to the appropriate authority such as an Incident Reporting System or supervisor/manager?
a) Definitely yes
b) Probably yes
c) Might or might not
d) Probably not
e) Definitely not 


\section{CASE \#5}

Karen Anderson had been diagnosed with congestive heart failure for many years. She took a number of medications including a ACE (angiotensin converting enzyme) inhibitor, beta blockers to slow progression of the heart failure and improve survival, diuretics to treat the fluid overload, and digoxin for control of her symptoms. In addition, she took a potassium supplement. Mrs. Anderson was 82 years old, thin, and lived alone. She was hospitalized in the morning after seeing her doctor for increased shortness of breath. Her physical exam revealed rales, swollen ankles, and a 6-pound increase in weight. Dr. Mason ordered a onetime dose of Lasix 20mg IV on admission followed by a lab draw to check electrolytes two hours later.

The nurse who assumed care of Mrs. Anderson had a heavy patient load that day. She gave the Lasix four hours after Mrs. Anderson arrived and after the lab had drawn the blood for the electrolyte panel. When Dr. Mason came in at $4 \mathrm{pm}$ to check on Mrs. Anderson, she noted the lab report indicated that the potassium level was low. Dr. Mason assumed that the blood was drawn after Mrs. Anderson had received the Lasix. Thus, she ordered $40 \mathrm{mg}$ of potassium and Lasix $20 \mathrm{mg}$ IV. The evening nurse noted the orders and decided to give the potassium after dinner. She gave the Lasix before dinner.

During dinner, Mrs. Anderson suddenly felt light-headed and called the nurse. She lost consciousness and a code was called. She was found to be in ventricular tachycardia and successfully converted with lidocaine and transferred to the ICU.

\section{Did an error occur?}
a) Yes
b) Maybe
c) No

Would you report this to the appropriate authority such as an Incident Reporting System or supervisor/manager?

a) Definitely yes

b) Probably yes

c) Might or might not 
d) Probably not

e) Definitely not

Do you think your colleagues would report this case to the appropriate authority such as an Incident Reporting System or supervisor/manager?

a) Definitely yes

b) Probably yes

c) Might or might not

d) Probably not

e) Definitely not

\section{Case \#6}

A physician ordered a patient to be started on a heparin drip. The nurse pulled the weight-based standing orders and had the MD identify the loading dose and rate per hour to run the heparin drip. By policy the facility requires that all heparin orders must be double-checked by another licensed professional before the medication is given to the patient. The RN drew up the loading dose and the amount needed to mix the heparin drip (20,000 units in 1 liter of NS).

All of this was completed per protocol. The nurse gave the bolus and initiated the heparin drip. At the bedside the nurse inadvertently set the pump at the wrong rate and did not discover the error until the 6-hour post heparin infusion PTT was due. The patient's INR and PTT were at panic values. The patient subsequently needed Vitamin $\mathrm{K}$ and fresh frozen plasma.

Did an error occur? 

a) Yes
b) Maybe
c) No

Would you report this to the appropriate authority such as an Incident Reporting System or supervisor/manager?
a) Definitely yes
b) Probably yes
c) Might or might not
d) Probably not
e) Definitely not

Do you think your colleagues would report this case to the appropriate authority such as an Incident Reporting System or supervisor/manager?
a) Definitely yes
b) Probably yes
c) Might or might not
d) Probably not
e) Definitely not

(Optional) Comments regarding this or any of the previous case studies 


\section{CURRICULUM VITAE}

Whitney Thomas Rogers, PhD, MPH

\section{Contact Information:}

Department of Neuroscience Training

University of Louisville

502/851-7445 (mobile)

522 East Gray Street

whitney.rogers@louisville.edu

Louisville, KY 40202

\section{EDUCATION}

University of Louisville, School of Public Health, Louisville, KY

- Doctor of Philosophy (PhD), May 2020

- Health Promotion \& Behavioral Sciences

- Dissertation Title: Converting Threats into Opportunities. Predicting Medical Error Reporting Behavior

- GPA $3.8 / 4.0$

University of Louisville, School of Public Health, Louisville, KY

- $\quad$ Master of Public Health (MPH), May 2013

- Epidemiology

- $\quad$ GPA: $3.8 / 4.0$

University of Louisville, School of Arts \& Sciences, Louisville, KY

- Master of Arts (MA), Clinical Psychology, December 2010

- Health Psychology

- GPA: $3.8 / 4.0$

University of Louisville, School of Arts \& Sciences, Louisville, KY

- Bachelor of Arts (BA), Biology, December 1995

- Major: Biology

- GPA: $3.4 / 4.0$

\section{RESEARCH \& WORK EXPERIENCE}

\section{2 - Present}

University of Louisville, Department of Neuroscience Training, Program Manager Program manager for the Kentucky Biomedical Research Infrastructure Network (KBRIN) and unit business manager (UBM) for the Department of Neuroscience Training on the UL Health Sciences Campus. The KBRIN is an INBRE program funded by a grant from the National Institute of General Medical Sciences of the National Institutes of Health (P20GM103436) to enhance biomedical research capacity, expand, and strengthen the research capabilities of biomedical faculty, and provide access to biomedical resources for promising undergraduate students throughout the eligible states. Annual federal funding $\$ 3.5$ million ( $\$ 81$ million in total awarded funds to date). 
Responsibilities include the financial management and administration of the overall program budgets and fiscal activities according to both Institutional and Federal policies and regulations. Develop and analyze budgets, monitor expenses, and implement budget changes as necessary. Responsible for internal program evaluation, which include tracking, monitoring through the use of National Student Clearinghouse data. Manage reporting functions including financial status reports, annual reports and reporting to both internal and external parties. Respond to internal and external audits. Supervise staff and provide human resource functions for the department.

Shared oversight of conference planning and summer programs including external advisory committee, statewide steering committees, Bioinformatic Summit committee, and summer program committee. Cultivate \& maintain effective working relationships with University Departments \& Schools, Kentucky Higher Education Institutions, \& Funding Agency Personnel.

\section{0-2012}

\section{University of Louisville, Department of Neurology, Program Manager}

Program Manager for the University of Louisville's Nationally-designated 'Center of Excellence' in Parkinson's disease research and care. Developed partnerships and relationships with community and grassroots organizations to raise awareness of neurological disorders within the Louisville, Lexington and Bardstown area. Conducted community needs assessment to develop outreach programs and services. Developed and coordinate training programs for professional and ancillary staff on Parkinson's disease and related neurological disorders. Directed community health programs including educational programs and symposiums, support groups and training. Prepared comprehensive reports detailing project initiatives, progress and outcomes. Supervised, hired, and evaluated personnel. Recruited and trained community volunteers. Served as liaison with granting agencies, research centers, community groups and higher education organizations.

Served as Co-PI on a Signature Partnership Initiative grant to better understand barriers to medical care and impediments to participation in medical research. Identify and conduct community-based focus groups with key informants and local residents. Collect and analyze data using multivariate statistical analyses and concept mapping methodology. Prepare and present research findings at meetings and symposiums. Develop, prepare and submit grant applications. Provide overall coordination and facilitation of research activities, budgeting, contract negotiation, expenditure monitoring, invoicing, planning, implementation and evaluation

\section{7-2010}

\section{University of Louisville, Department of Psychological and Brain Sciences, Graduate Research Assistant}

Graduate Research Assistant for the Health Behavior Change Research Program.

Supervised and trained undergraduate lab members and junior colleagues. Collaborated in the design and development of projects by providing advice, guidance and recommendations on the practicality and feasibility of intervention programs and data collection efforts. Train medical health care providers and colleagues on psychological and behavioral interventions designed to improve health outcomes. Constructed statistical models to explain, predict and analyze patterns of behaviors and outcomes. Prepared reports, manuscripts and posters for dissemination. Assisted in website design and maintenance. 


\section{3-2007}

University of Louisville, Department of Neurology, Clinical Research Coordinator Managed all aspects of clinical and research studies for the UL Movement Disorder Program, including investigator-initiated, industry and federally-sponsored clinical trials. Served as liaison between the investigators, health care providers, regulatory staff, internal or external sponsors, and participants. Negotiated contracts and developed budgets. Assisted in the development of training modules (SOP/MOP). Assisted with grant preparation and submission for extramural funding opportunities. Conducted literature reviews, analyzed preliminary pilot data, and drafted reports.

Project Title: Genetic and Environmental Risk Factors for PSP. A multi-center, case-control study on genetic and environmental risk factors associated with mitochondrial dysfunction in a rare progressive neurodegenerative disease, Progressive Supranuclear Palsy (PSP). PI: Litvan, I. Funding Period: 2006-2013

Funding Source: National Institute of Health, National Institute on Aging (5R01AG024040) Total Funding: \$3.4 million

Role: Clinical Research Coordinator (Multi-Site Lead)

Project Title: Genetic Linkage Study in Parkinson's Disease (GENEPD). Multicenter study on siblings and other primary related families with Parkinson's disease. PI: Slevin, J., Litvan, I.

Funding Period: 2006-2013

Funding Source: National Institute of Health, National Center for Research Resources (5M01RR002602)

Role: Clinical Research Coordinator (Site)

Project Title: Longitudinal Study on PSP. A 5-year, longitudinal study on the course and progression of Progressive Supranuclear. PI: Litvan, I.

Funding Period: 2006-2013

Funding Source: Society for Progressive Supranuclear Palsy/'Cure PSP'

Role: Clinical Research Coordinator (Multi-Site Lead)

Project Title: Energy Metabolism in Neurodegenerative Diseases: A Randomized, Double Blind, Placebo-Controlled Clinical Pilot Trial of Pyruvate, Creatine, and Niacinamide in Progressive Supranuclear Palsy. PI: Litvan, I.

Funding Period: 2004-2009

Funding Source: Society for Progressive Supranuclear Palsy/'Cure PSP'

Role: Clinical Research Coordinator

Project Title: Psychogenic Movement Disorders, ERP Study. PI: Litvan, I., Schoenbachler, B., Chand, P.

Funding Period: 2004-2009

Funding Source: Investigator Initiated/ EVPRI Funded

Role: Clinical Research Coordinator 
Project Title: Cytokines and Inflammation in Atypical Parkinsonian Disorders. PI: Litvan, I.

Funding Period: 2004-2009

Funding Source: Investigator Initiated/ EVPRI Funded

Role: Clinical Research Coordinator

\section{CLINICAL EXPERIENCE}

2008-2010

Graduate Student Therapist, Noble H. Kelley Psychological Services Center, Department of Psychological and Brain Sciences, University of Louisville, KY. Integrative Biopsychosocial approach to the assessment and treatment of psychological disorders. Specific experience includes working with mentally and physically disabled, transgendered and personality disordered clients in addition to individuals with severe mental illnesses. Additional instructional training in Cognitive Behavioral therapy, Dialectical Behavior therapy and Narrative therapeutic techniques. Supervisor: Jay Irby, Ph.D.

\section{7-2008}

Graduate Student Therapist, Veterans Affairs (VA) Medical Center and Noble H. Kelley Psychological Services Center, Department of Psychological and Brain Sciences, University of Louisville Louisville, KY. Member of the Health Psychology team providing assessments and interventions for veterans through a collaboration with the Home-Based Primary Care, Geriatrics and Extended Care department of the VA hospital. Responsibilities included providing a Cognitive Behavioral approach to the assessment and treatment of psychological and healthrelated disorders. Specific interventions included smoking cessation, depression and anxiety associated with chronic diseases and co morbidities, post-traumatic stress disorders, communication strategies and personality disorders. Additional instructional training in Dialectical Behavior therapy. Supervisor: Barbara Stetson, Ph.D.

\section{3-2007}

Clinical Assistant, University of Louisville, Department of Neurology, Division of Movement Disorders. Multidisciplinary approach to treating individual with neurological and psychological impairments. Responsibilities included providing comprehensive assessments and neuropsychological testing. Assessments were focused on obtaining medical histories, identifying physical and social needs, obtaining referrals for resources, medication adherence and chronic disease management. Neuropsychological evaluations were conducted to assess for dementia, frontal-lobe dysfunction, depression and quality of life. Supervisor: Irene Litvan, M.D.

\section{PUBLICATIONS \& PRESENTATIONS}

\section{Publications}

Kelley, K., Peavy, G., Edland, S., Rogers, W., Riley, D., Bordelon, Y., Standaert, D., Reich, S., Litvan, I. (2017). The Role of Stress as a Risk Factor for Progressive Supranuclear Palsy. J Parkinsons Dis. 2017;7(2):377-383.

Stetson, B., Schlundt, D., Rothschild, C., Rogers, W., Floyd, J.B., Mokshagundam, S.P., Krishnasamy, S. (2010). Reliability and Validity of a Theoretically-Based Diabetes SelfManagement Assessment Tool. Society of Behavioral Medicine, Seattle, WA. 
Rogers, W., Meyer, J., Rothschild, C., Bonner, J., Das, N., Richardson, K., Mokshagundam, S.P., Krishnasamy, S., Kong, M., Stetson, B. (2009). Social isolation is associated with geographic socioeconomic status and quality of life in at-risk, underserved adults with type 2 diabetes. Annals of Behavioral Medicine, 37, s199.

Richardson, K., Rogers, W., Bonner, J., Rothschild, C., Meyer, J. Das, N. Mokshagundam S.P., Krishnasamy, S. Kong, M., Stetson, B. (2009). Geographic socioeconomic status, health literacy and perceived barriers to self-care in at-risk, underserved adults with type 2 diabetes. Annals of Behavioral Medicine, 37, s197.

Meyer, J. A., Bonner, J., Rothschild, C., Rogers, W., Foster, M., Richardson, K., Robertson, E., Reitz, P., \& Stetson, B. (2008). Acceptance and experiential avoidance: Associations with quality of life and self-care in adults with type 2 diabetes. Annals of Behavioral Medicine, 35, (Supplement 1), S46.

\section{Editorial Experience}

Editorial Assistant for published book. “Atypical Parkinsonian Disorders”, Litvan, I. (Ed.) Humana Press (2005).

\section{Presentations}

Rogers, W., Young, B., Chaney, C., Jones, G., Ayangeakaa, S., Muvuka, B., LaJoie, S. (2015). Redesigned medication labels better communicate information to patients. Research! Louisville.

Rogers, W. (2010). Cluster A Personality Disorders: Etiology and Treatment. Presentation at the University of Louisville, Department of Psychological and Brain Sciences, Louisville, KY.

Rogers, W., Floyd, J.B. (2010). Diabetes Self-Management: Theoretically-based Behavioral Interventions. Presentation at the University of Louisville, Department of Psychological and Brain Sciences, Louisville, KY.

Rogers, W. (2009). Biological Basis of Development. Presentation at Presentation at the University of Louisville, Department of Psychological and Brain Sciences, Louisville, KY.

Rogers, W., Crawford, N., Waford, R., Robertson, C., Daup M. (2009). Integrative Psychotherapy: Case Conference. Presentation at the University of Louisville, Department of Psychological and Brain Sciences, Louisville, KY.

Allison, K. E., Flores, P., Gordon, M., Hess, L., Rogers, W., \& Zimmerman, J. (2008). Maintaining healthy behavior: How to stick with healthy eating. Presentation at Morgan \& Pottinger PSC, Louisville, KY.

Rogers, W. Smoking Cessation. (2008). Presentation to the VA team at the University of Louisville Psychological Services Center. Louisville, KY.

\section{TEACHING EXPERIENCE}


Graduate Teaching Assistant, Advanced Graduate Statistics II. Professor/Supervisor: Pavel Zahorik, Ph.D. (Spring 2009, Spring 2010)

Graduate Teaching Assistant, Advanced Graduate Statistics I. Professor/Supervisor: Cara Cashon, Ph.D. (Fall 2008, Fall 2009).

Graduate Teaching Assistant, Undergraduate Personality. Professor/Supervisor: Richard Lewine, Ph.D. (Spring 2008)

Graduate Teaching Assistant, Introduction to Psychology. Professor/Supervisor: Melissa Rowe, Ph.D. (Fall 2007). Graduate Teaching Assistant, Life Span-Developmental Psychology.

Professor/Supervisor: Melissa Rowe, Ph.D. (Fall 2007)

Substitute Teacher, Jefferson County Public School System, grades 1-12. Part-time, paid employment (1992-2003)

English Instructor. NOVA Corporation, Boston, MA. 1-Year assignment - Tokyo, Japan: English as a Second Language (ESL) instructor. Full-time, paid, international employment (19961997)

\section{VOLUNTEER AND COMMUNITY SERVICE EXPERIENCE}

Habitat for Humanity. Volunteer / Worker (2001-2003)

Amigos de los Partidos. Guadalajara, Mexico. Medical Mission/Volunteer (1993)

Kentucky Humane Society. Animal Care Volunteer (1992)

\section{SKILLS:}

SPSS - Highly Proficient

Tableau - Proficient

Microsoft (Word, PowerPoint, Excel, Access) - Highly Proficient

PeopleSoft - Highly Proficient

Endnote - Proficient

Adobe Creative Suite - Proficient 